\title{
Analysis of lateral openings in tunnel linings
}

\author{
Master thesis (draft) \\ for the completion of the Master's Curriculum: "Projektkoordination im \\ Tunnelbau"
}

Panagiotis Spyridis

Supervisor: Prof. DDI DDr K. Bergmeister

Department of Civil Engineering and Natural Hazards

Institute of Structural Engineering (IKI)

Vienna, March 2013 


\section{Contents}

Page number

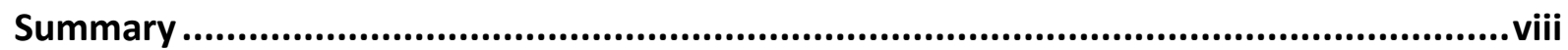

Kurzfassung ...................................................................................................................viii

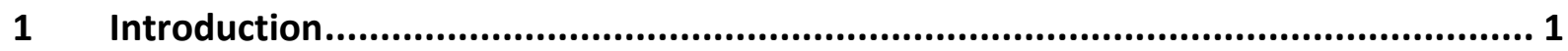

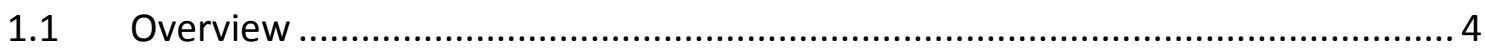

2 State of the art....................................................................................................... 5

2.1 Sprayed concrete tunnel lining structures.................................................... 5

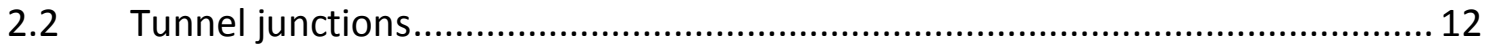

2.3 Numerical modeling and application in tunneling ........................................... 23

$3 \quad$ Investigation approach \& methodology .................................................................. 33

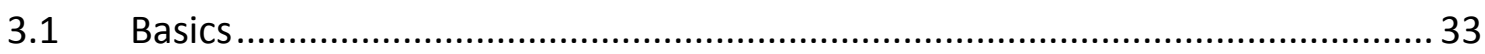

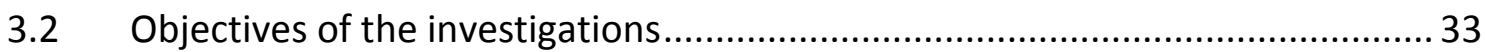

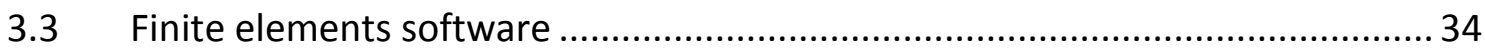

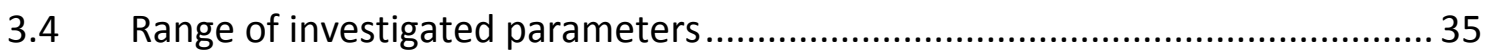

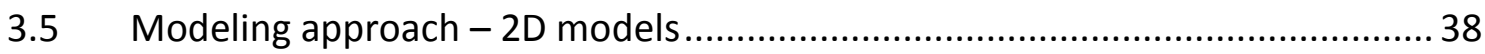

3.6 Modeling approach - 3D models ................................................................. 41

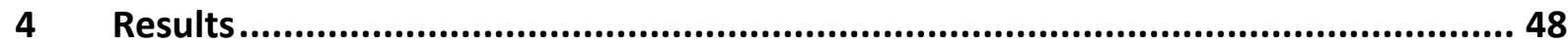

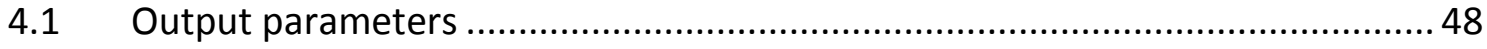

4.2 Typical results and interpretation ................................................................. 48

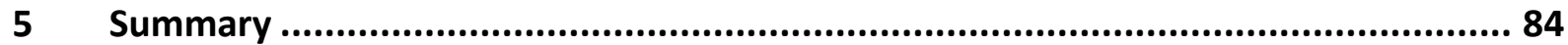

5.1 Main conclusions and recommendations for design .......................................... 85

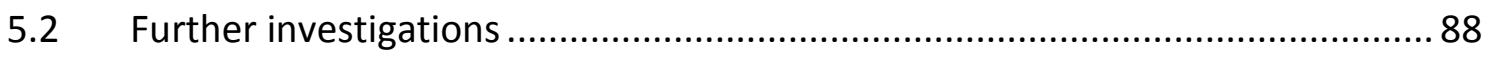

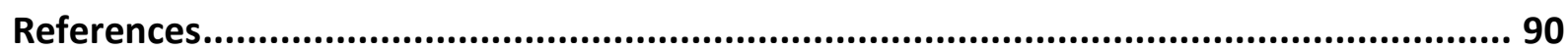




\section{List of figures}

Figure 1: Layout of Tottenham Court Road Station in London (from www.constructionenquirer.com)

Figure 2: Layout of Brenner Base Tunnel (from: www.tunneltalk.com)

Figure 3: Natural flow model to represent flow redistribution around obstacles (thanks to Despoina Kalapoda of Foster \& P. for this sketch) 15

Figure 4: Notation of Kirsch formulas for stress assessment around circular openings

Figure 5: Strut-and-tie model for stress distribution around a circular opening in a plate $\ldots . .17$

Figure 6: Arch model for a simplified 2D assessment of hoop forces in a circular tunnel ..... 18

Figure 7: Simplification/idealization of static systems (down) responding to soil-structure interactions at characteristic sections (up) around a tunnel opening

Figure 8: 3D Junction geometry (left) and actual junction projected/developed shape (right) for the case of two circular tunnels; aspect ratio is 0.9

Figure 9: Geometry of the circular segment (cross section of the parent tunnel, left), and of the circular disk (cross section of child tunnel, right).

Figure 10: Graphical representation of Mohr - Coulomb failure criterion 28

Figure 11: Graphical representation of linear-elastic perfectly-plastic concrete damaged plasticity material constitutive law

Figure 12: Illustrative description models as idealized abstractions of reality, neglecting certain aspects of the project's environment

Figure 13: Typical examples of different approaches for numerical analyses depending on the structural element type

Figure 14: Definition of 3D modeling geometric parameters and notation (d: child Tunnel diameter, D: parent tunnel diameter, $\mathrm{H}$ : tunnel centerline depth)

Figure 15: Indicative geometry of a 2D plate model (for child/parent tunnel diameter $=0.9$ ) 
Figure 16: Indicative mesh of a 2D plate model; highlighted lines indicate regions with local increased mesh density

Figure 17: Load application for a 2D plate model.....

Figure 18: Overview of soil block for the 3D models

Figure 19: Overview of the 3D models including the tunnel structure, showing the lined tunnel in red

Figure 20: Detailed view of the modeled tunnel lining in the 3D model

Figure 21: Overview of the mesh discretization of the soil block for the 3D models

Figure 22: Overview of the mesh discretization of tunnel lining for the 3D models; purple indicates regions with local increased mesh density.

Figure 23: Detailed mesh plots of one of the 3D models with discretization refinement inside the tunnel lining

Figure 24: Overview of boundary conditions for one of the 3D models .47

Figure 25: Overview of sets for one of the 3D models

Figure 26: Notation of monitored section forces around the openings

Figure 27: Model Nr.10: 2D-090; SF1: Longitudinal forces 52

Figure 28: Model Nr.10: 2D-090; SF2: Hoop forces 52

Figure 29: Model Nr.26: 2D-075; SF1: Longitudinal forces 53

Figure 30: Model Nr.26: 2D-075; SF2: Hoop forces 53

Figure 31: Model Nr.42: 2D-060; SF1: Longitudinal forces 54

Figure 32: Model Nr.42: 2D-060; SF2: Hoop forces 54

Figure 33: Comparison of 2D FE models with Kirsch solution; Hoop Forces at Crown and Invert (SF2-Cl) 55

Figure 34: Comparison of 2D FE models with Kirsch solution; Longitudinal Forces at Crown and Invert (SF1-CI) 55

Figure 35: Comparison of 2D FE models with Kirsch solution; Hoop Forces at Springlines (SF2SP). 56 
Figure 36: Comparison of 2D FE models with Kirsch solution; Longitudinal Forces at Springlines (SF1-SP) 56

Figure 37: Model Nr.1: 3D-25-35-090; E11: Deformed shape and strains in longitudinal direction 58

Figure 38: Model Nr.1: 3D-25-35-090; E22: Deformed shape and strains in hoop direction.. 58

Figure 39: Model Nr.20: 3D-50-25-075; E11: Deformed shape and strains in longitudinal direction 59

Figure 40: Model Nr.20: 3D-50-25-075; E22: Deformed shape and strains in hoop direction 59

Figure 41: Model Nr.39: 3D-100-15-060; E11: Deformed shape and strains in longitudinal direction 60

Figure 42: Model Nr.39: 3D-100-15-060; E22: Deformed shape and strains in hoop direction 60

Figure 43: Model Nr.1: 3D-25-35-090; RelU: Relative absolute displacements (before and after breakout) 61

Figure 44: Model Nr.20: 3D-50-25-075; RelU: Relative absolute displacements (before and after breakout)

Figure 45: Model Nr.39: 3D-100-15-060; RelU: Relative absolute displacements (before and after breakout) 62

Figure 46: Model Nr.1: 3D-25-35-090; SF1: Longitudinal forces .65

Figure 47: Model Nr.20: 3D-50-25-075; SF1: Longitudinal forces .66

Figure 48: Model Nr.39: 3D-100-15-060; SF1: Longitudinal forces 66

Figure 49: Influence of soil Young's modulus on Longitudinal Forces at Crown/Invert (sf1-Clnorm)

Figure 50: Influence of child-parent tunnel aspect ratio on Longitudinal Forces at Crown/Invert (sf1-Cl-norm) .67

Figure 51: Influence of soil Young's modulus on Longitudinal Forces at Springlines (sf1-SPnorm) 68 
Figure 52: Influence of child-parent tunnel aspect ratio on Longitudinal Forces at Springlines (sf1-SP-norm) 68

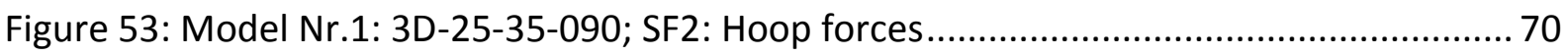

Figure 54: Model Nr.20: 3D-50-25-075; SF2: Hoop forces................................................. 70

Figure 55: Model Nr.39: 3D-100-15-060; SF2: Hoop forces. 71

Figure 56: Influence of soil Young's modulus on Hoop Forces at Crown/Invert (sf2-Cl-norm)

Figure 57: Influence of child-parent tunnel aspect ratio on Hoop Forces at Crown/Invert (sf2Cl-norm)

Figure 58: Influence of soil Young's modulus on Hoop Forces at Springlines (sf2-SP-norm) .. 73 Figure 59: Influence of child-parent tunnel aspect ratio on Hoop Forces at Springlines (sf2-SPnorm) .73

Figure 60: Model Nr.1: 3D-25-35-090; SM1: Longitudinal moments 74

Figure 61: Model Nr.20: 3D-50-25-075; SM1: Longitudinal moments .75

Figure 62: Model Nr.39: 3D-100-15-060; SM1: Longitudinal moments 75

Figure 63: Influence of soil Young's modulus on Longitudinal Moments at Crown/Invert (sm1Cl-norm) 76

Figure 64: Influence of tunnel aspect ratio on Longitudinal Moments at Crown/Invert (sm1Cl-norm) 76

Figure 65: Influence of soil Young's modulus on Longitudinal Moments at Springlines (sm1SP-norm) 77

Figure 66: Influence of child-parent tunnel aspect ratio on Longitudinal Forces at Springlines (sm1-SP-norm). .77

Figure 67: Model Nr.1: 3D-25-35-090; SM2: Hoop moments .78

Figure 68: Model Nr.20: 3D-50-25-075; SM2: Hoop moments. 79

Figure 69: Model Nr.39: 3D-100-15-060; SM2: Hoop moments 79 
Figure 70: Influence of soil Young's modulus on Hoop Moments at Crown/Invert (sm2-Clnorm)

Figure 71: Influence of child-parent tunnel aspect ratio on Hoop Moments at Crown/Invert (sm2-Cl-norm) 80

Figure 72: Influence of soil Young's modulus on Hoop Moments at Springlines (sm2-SP-norm)

Figure 73: Influence of child-parent tunnel aspect ratio on Hoop Forces at Springlines (sm2SP-norm) 81

Figure 74: Development of a tunnel with opening, and envelope of circular opening shapes to be assessed through analytical methods (e.g. Kirsch) 86 


\section{List of tables}

Table 1: Overview of most common analytical approaches for tunnel analyses (adopted from

[4])

Table 2: Continuum elements lining properties, corresponding to beam model properties of (i) Primary Lining: $E=15 \mathrm{GPa}, v=0.2, f_{c}=28 \mathrm{MPa}, f_{t}=0.5 \mathrm{MPa}$, (ii) Secondary Lining: $E=30 \mathrm{GPa}, \mathrm{v}=0.2, \mathrm{f}_{\mathrm{c}}=28 \mathrm{MPa}, \mathrm{f}_{\mathrm{t}}=2.0 \mathrm{MPa}$; adopted by $[12]$

Table 3: Recommended/Default parameters for the concrete damaged plasticity constitutive model, according to [12]

Table 4. Analyzed parameters (S.J.B. stands for Single Junction Breakout, i.e. only one child tunnel). Lines 10, 22, 34 correspond to the averaged values from the 3D models for aspect rations $d / D$ of $0.9,0.75$, and 0.60 respectively. The material properties of concrete are elastic, with Young's modulus $\mathrm{E}_{\mathrm{c}}=15 \mathrm{GPa}$ and Poisson's ratio $\mathrm{v}_{\mathrm{c}}=$ 0.2. Also see notation in Figure 14.

Table 5: Summary of results from the various analysis (also refer to Table 4 and Figure 26) 49

Table 6: Summary of normalized results (divided with average hoop force at ring closure) from the various analysis (also refer to Table 4 and Table 5) 50

Table 7: Graphical summary of the influences of the checked parameters 82 


\section{Summary}

The present study discusses the structural response of tunnels when a near circular breakout is performed laterally at their lining. In the first part, this thesis is set in its context, by providing some main concepts of tunneling design and construction, aspects of the numerical modeling with focus on underground structures, analytical solutions for openings in shells/plates from literature, and some main elements with respect to the layout and the descriptive geometry of the idealized investigated structural systems. Furthermore, the investigated systems and the description of the investigation methods is provided, this mainly being linear-elastic finite elements analysis, yet with inclusion of analytical solutions for reference and with some analysis assuming non-linear soil behavior in order to investigate particular aspects in the discussed systems. This thesis comes to contribute to the little hitherto available in literature with respect to the soil structure-interaction and the structural behavior at tunnel breakouts and junctions, while certain reference is given for future projects through 35 analyzed cases.

\section{Kurzfassung}

Die vorliegende Arbeit beschreibt die statische Beanspruchung von Tunnelbauwerken, bei denen ein kreisförmiger seitlicher Querschlag an der Tunnelauskleidung durchgeführt wird. Im ersten Teil werden wichtige Aspekte der Bemessung und Konstruktion von Tunnelsystemen, der Geometrie, sowie der statischen Idealisierung und Analyse des Systems erläutert. Darüber hinaus erfolgt die Beschreibung der Untersuchungsmethoden, die im wesentlichen auf linear-elastischen Finite-Elemente-Analysen, unter Miteinbeziehung analytischer Lösungen und zwei nicht-linear-elastischen Ansätzen, basieren. Im Rahmen der vorliegenden Arbeit wurden rund 35 Fallbeispiele untersucht. Diese Arbeit stellt einen wichtigen Beitrag zur Boden-Bauwerk-Interaktion und der statischen Beanspruchung von Tunnelquerschläge dar. Nachdem diese Forschungsgebiete in der Literatur unterrepräsentiert ist, kann diese Arbeit als Referenz für zukünftige Bemessungsprojekte herangezogen werden. 


\section{$1 \quad$ Introduction}

Modern tunneling is usually confronted with geometrically complex excavation layouts. This poses a huge challenge to the engineering society in design and construction, and it involves advanced requirements in terms of material properties and durability, time and cost logistics, impacts on third parties, as well as insurance and contracting issues. Complex geometrical layouts of underground excavations are typically dealt with by use of sprayed concrete support. This technique is often referred to as the New Austrian Tunneling Method (NATM), the Sequential Excavation Method (SEM), or the Sprayed Concrete Lining (SCL) method. This thesis comes to provide new insights to the structural design of lateral breakouts and openings at tunnels with focus on sprayed concrete linings. This is often a case in the design of cross passages or underground metro/rail stations. As an example from the Crossrail Project, the following number of openings appears in each station: Bond Street: 24; Tottenham Court Road: 22; Liverpool Street: 24; Whitechapel: 20. The entire geometry of the Tottenham Court Road station (London Underground and Crossrail) is presented in Figure 1. Lateral breakouts are also typically formulated at cross passages that connect the two bores of long railway or highway tunnels, mainly for evacuation purposes. The geometry of the Brenner Base Tunnel serves as an example in Figure 2.

Motivation for this study arises from the involvement of the author in the design of the Crossrail project SCL stations in London from the side of the independent (Category 3) design checker. During this work the requirement for the analysis of tunnel junctions and the assessment of a tunnel lining once an opening is carried out in the constructed and loaded lining of near-circular tunnels. This experience dictated the necessity for an adequate simulation of this process, whereby it has often become a question whether an empirical, an analytical, a 2D numerical solution, or a 3D numerical solution is most cost-efficient. The resources required for empirical or analytical approaches are typically low and may prove sufficient for smaller openings, i.e. openings with a diameter quite smaller than the one of the "parent" tunnel. Yet for larger openings, a strong re-distribution of stresses is expected in the lining during a breakout and an analysis with a higher precision is pursued. Given the critical geometry of the structures in this case, the engineer may resort to the implementation of numerical analyses. The choice between the two- or three-dimensional 
numerical approaches then comes to play. In those cases the solution is tailored, so the detailed geometry and other project-specific characteristics, as for example the ground and lining material properties, are taken into account appropriately.

The intention and consequent scope of the present study is (a) to show the basics of modeling, (b) to present the sensitivities of the respective analytical, two-, and threedimensional Finite Element Analyses (FEA) to strategically selected input-parameters, and (c) to compare these approaches and provide feedback for the design and construction of tunnel openings at junctions. The basis of the study comprises mainly numerical calculations, yet overlaid with the author's hands-on appreciation of the discussed aspects.

The area of investigation is limited to cases pertaining to tunnel structures with the following characteristics:

- Circular sections for both the parent and the child tunnel

- Soft substrate, and ground model envisaging shallow tunnels

- Elastic behavior of both the soil and the lining material

Still, it is anticipated that this work covers a wide range of tunneling systems relevant to practice and adds to the general understanding of their behavior.

Significance of the presented investigation lies mainly in the facts that

- The effects of essential parameters in tunnel junction analysis and design are presented.

- An educated selection between analytical, two-, and three-dimensional methods of analysis is nourished.

- A set of calculated stress patterns around tunnel openings is made available for reference among designers and for future research in the field. 


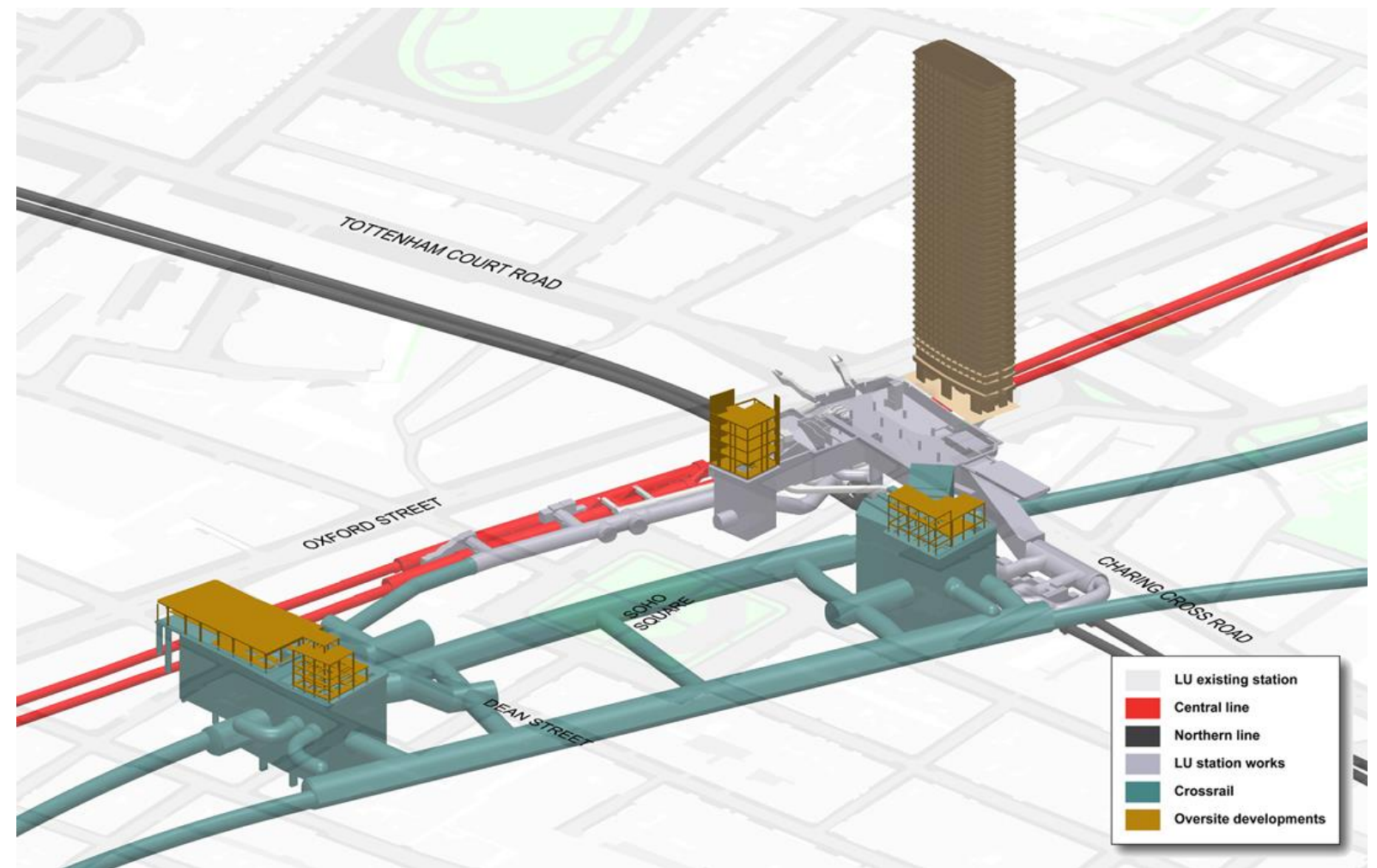

Figure 1: Layout of Tottenham Court Road Station in London (from www.constructionenquirer.com)

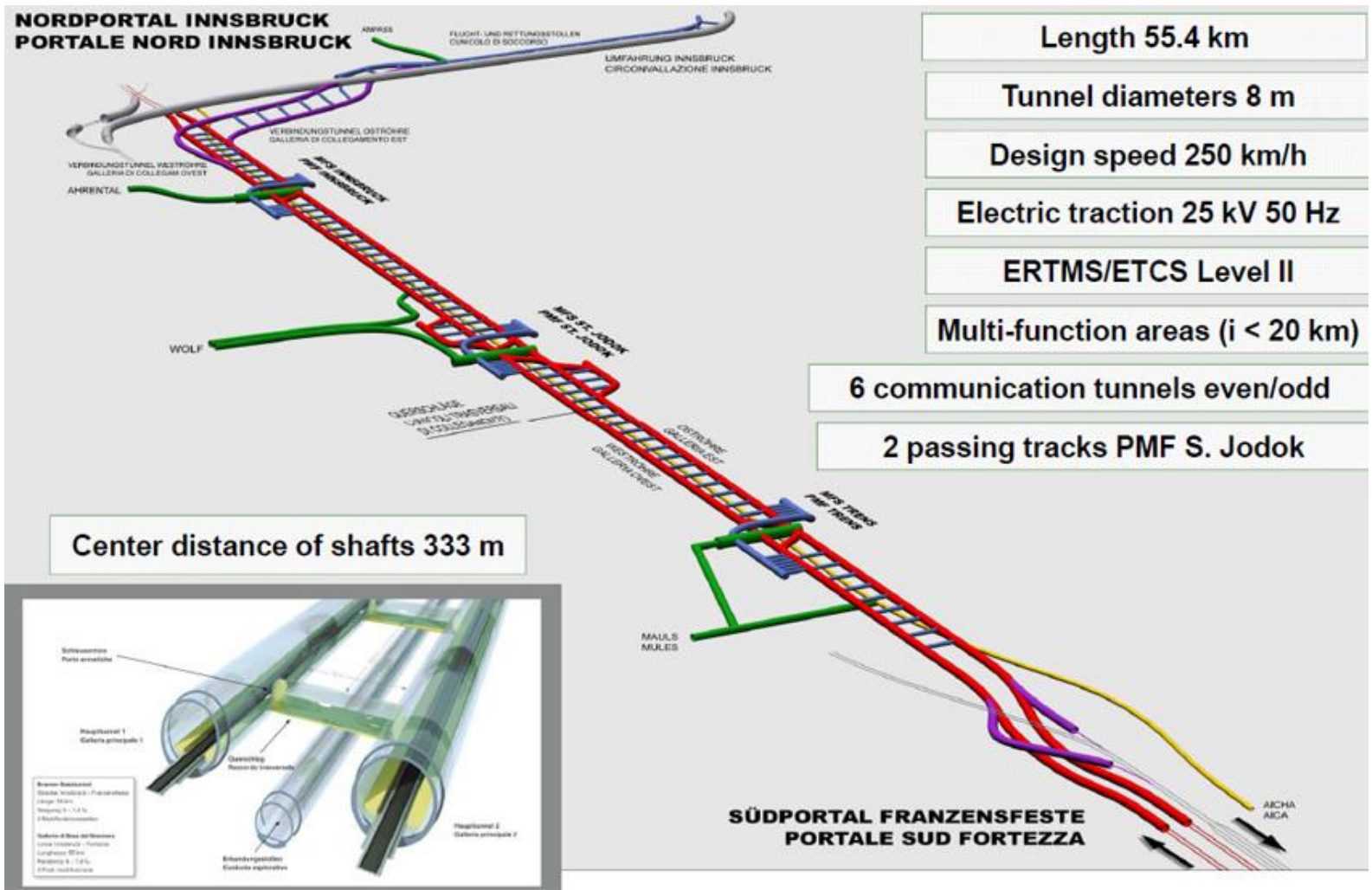

Figure 2: Layout of Brenner Base Tunnel (from: www.tunneltalk.com) 


\subsection{Overview}

A preview of this thesis' structure is given below in the form of bullet points.

Chapter 1 Introduction: Overview; motivation; intention, scope, and limitations; research significance.

Chapter 2 State-of-the-art: Relevant tunnel construction methods; FEA; relevant previous studies.

Chapter 3 Investigation approach and methodology: Concept and methods of the numerical analyses.

Chapter 4 Results and interpretation: Discussion of results, identification of influences, comparisons of results using different approaches.

Chapter 5 Summary: Conclusions, recommendations, discussion of potential further investigations. 


\section{State of the art}

\subsection{Sprayed concrete tunnel lining structures}

In the present study sprayed concrete linings are mainly assumed. Sprayed concrete is typically applied for mined underground structures (tunnels, caverns, shafts). Especially in the case of junctions to cross passages, SCL tunneling often lends itself, due to the several advantages it carries along regarding the adaptability in structure geometries. The basic background of this technique, and elementary design criteria and a generic design approach for such structures are given below. Furthermore construction aspects of sprayed concrete tunnels and lateral breakouts are provided.

\subsubsection{Main definition of sprayed concrete tunnel lining structures}

In the present context, sprayed concrete tunnels are mined tunnel structures where the construction method is open face sequential excavation. Various excavation sequences may be used, while their applicability is dependent primarily on the geological conditions and the tunneling induced deformation/settlement regulation requirements. These sequences may be:

- Full face excavation

- Multiple face excavation, i.e. face advance divided to Top-Heading, Bench and Invert Excavation

- Inclined full face excavation

- Partial ring closures, i.e. implementation of pilot tunnels, temporary inverts, temporary sidewall drifts.

These types of structures are typically excavated and constructed with conventional equipment as opposed to tunnels excavated by use of boring machines. Slightly different concepts govern mined tunnels in rural and urban environment. Still it may be assumed that the two cases entail common technical aspects, e.g. sequential excavation with conventional 
equipment, observational approaches, preparation and maintenance of additional support/toolboxes during construction. The design approach can also be varied depending on whether the substrate is rock/soft rock (where the rock excavation self-carrying capacity is mobilized), or soft ground and shallow/urban tunnels.

The investigation presented herein mainly refers to shallow SCL tunnels. The analyses are based on a "wished-in-place" approach (explained further in 3.6) which by its nature simplifies the excavation simulation. This means that this type of analysis may approximate different excavation sequences used in mined tunnels, but it can even represent tunnels realized through mechanized excavation with e.g. a tunnel boring machine (TBM).

\subsubsection{Material - Sprayed Concrete}

The use of sprayed concrete lining systems is becoming increasingly common in soft ground because of the flexibility that $\mathrm{SCL}$ offers in terms of the shape of the tunnel and the combination of support measures. Two-pass systems have traditionally been used in SCL tunnels where the ground is not largely self-supported. The primary support systems are designed to maintain a stable excavation so that a permanent lining can be placed. In soft ground projects, single layer SCL is rarely used because of concerns over the durability of the lining and waterproofing issues. In rock tunnels, sprayed concrete is used at least as the initial support installed right after excavation in order to stabilize the excavated area for construction health and safety purposes. Occasionally the lining contains lattice girders and mesh reinforcement. However, a recent trend appears to be steel fiber reinforcement for various reasons (e.g ductility in tension, stand-up strength in fresh conditions, crack control, and improved shrinkage behavior). Sprayed concrete in tunneling is typically applied in the form of wet-mixes for various reasons, among which are health and safety requirements in the underground working space (e.g. dust reduction), reduced material consumption (rebound reduction), and better material properties (early age strength, adhesion, uniform quality).

The main constituents of sprayed concrete liners are listed below. The mix design can substantially affect the time-dependent strength development and structural response of sprayed concrete. 
- cement,

- water

- aggregates (they should be checked for their susceptibility to alkali-silica reaction),

- fly ash (increases strength, chemical resistance and durability, and pumpability, while reducing the required cement content)

- silica fume (increases pumpability, cohesiveness and adhesion)

- chemical admixtures (e.g. accelerators, plasticizers and superplasticizers). The admixtures are used to improve mainly logistics-related properties of shotcrete, i.e. to increase the stiffening rate, to produce a fast set and provide early strength development, to minimise the amount of water in the mix, to maintain workability/pumpability and extend the open transportation time.

In most cases of underground constructions sprayed concrete has proven to provide beneficial properties when reinforced with fibers. Fibers are added to improve the following properties:

- Tensile and flexural strength

- Thermal and shrinkage cracking

- Abrasion and impact resistance

- Ductility and toughness

- Early age stability

- Fire resistance

\subsubsection{Types of Support}

Dual Shell: This is the most common support technique currently on worldwide reach. In this case the primary lining / primary support bears and distributes the temporary loads, these being mainly due to mobilization of the ground. Stiff primary supports (e.g. thick linings) are pursued for minimization of ground deformations as for example in urban shallow tunnels. In cases where the rock mass provides adequate inherent strength, thinner supports are possible. The secondary lining comes to resist long term ground loads and 
water pressure, and actions initiated inside the tunnels during operation (e.g. catenary loads or impact/accidental actions). It has to be noted, that in the present study only primary lining in short-term loading conditions is considered.

Composite shell: In this case the primary lining is assumed to participate in the long term load bearing system together with the secondary lining. Analysis of composite shells is reliant on specified shear resistance in the primary and secondary lining support interfaces exists. The waterproofing system is typically located in this interface. Composite shells have a potential to slightly decrease the excavation but then a link of the temporary and permanent works, design, and design life needs to be considered. As mentioned above, the present study does not account for a secondary lining.

Other planned support measures or optional "toolbox items" may be used, mainly in conjunction with the primary support in order to decrease deformations and increase safety conditions in the excavation. Such measures essentially comprise various forepoling/spiling, and grout-umbrella (arching) techniques, as well as rockbolt (tensioned) or dowel (untensioned) elements. Besides, geotechnical engineering techniques (e.g. compensation grouting, jet grouting, dewatering) may be carried out to modify the properties of the ground or groundwater, either permanently or temporarily to make tunneling safer or control ground movements. The effect that these methods have on the ground properties, the tunnel loading, the environment, and third-party assets are accounted for in the course of the design.

\subsubsection{Considerations for waterproofing}

In the typical two-layer concrete shell system, the waterproofing system is installed inside the primary lining, possibly on top of a smoothing or regulating layer. There are two major waterproofing systems:

- A closed or 'tanked' system in which the entire underground structure is wrapped with a waterproofing medium, and as such, is disconnected from the in-situ water situation. This will subject the next layer inside the waterproofing (final/secondary lining) to prevalent water pressure. For tanked waterproofing 
systems, two types of products are being used, namely (i) sheet membrane waterproofing systems and (ii) spray-on waterproofing systems. Watertight concrete can also serve a similar function.

- An open waterproofing system, or so-called 'umbrella system', introduces a waterproof layer only in the crowns and sides of the tunnel. In the invert, the occurring water is directed into drainage pipes and brought to the surface. This system may have a permanent impact on the ground hydrology and be associated with higher maintenance costs but in general this system eliminates the need for design combinations including water pressure.

\subsubsection{Considerations for the long-term structural behavior}

Special design assumptions should indicate the load sharing between the two linings (primary and secondary) in the long term. Typically in the dual shell design, the primary lining is assumed fully degraded in the long term, whereas in composite lining systems a combined primary-secondary lining structural response is envisaged. The design assumptions for the participation of the primary lining in the tunnel structure's load bearing behavior on the long term should be based on verification of the long term waterproofing/interface structural properties and the degradation of the primary lining. In the present case, the analyses are based on short term situations and the secondary lining is neglected.

\subsubsection{Considerations for instrumentation and monitoring}

An integral part of the conventional/mined tunneling method is the verification of design assumptions made regarding ground-structure interaction as a response to the excavation process by means of in-situ monitoring. Monitoring before, during \& after construction is a prerequisite of this technique and allows for assessments of environmental impacts (e.g. ambient ground movements and settlements), and deformations to ensure the design validation. The instrumentation aims at a detailed and systematic measurement of deflection of the primary lining, stresses in the lining, stresses between the lining and the 
ground, surface settlements, and deformations of third-party assets. Monitoring regarding the excavation behavior, the tunnel structural performance, and the analysis and design verification forms part of the tunnel design. Based on this information immediate decisions can be made on site concerning proper excavation sequences and adequacy of the primary support. Models with validated results may also be used in life-cycle-engineering activities throughout the structure's lifetime.

Surface Instrumentation: The general instrumentation may include surface settlement markers where appropriate, cased deep benchmarks, subsurface shallow and deep settlement indicators, sliding micrometers, inclinometers, borehole extensometers, and piezometers. The locations, types and number of these instruments are normally determined by consultations between the design teams of different disciplines to provide information on surface settlements and subsurface structure deformations, and to compliment the instrumentation readings.

Tunnel Instrumentation: Three different characteristics of the ground/lining response to the excavation and load redistribution are typically observed using special monitoring devices:

- the deformation of the primary lining and surrounding ground;

- axial stresses acting within the primary lining;

- radial stresses acting as "ground load" upon the primary lining;

Interpretation of monitoring data with respect to the verification of design parameters are then evaluated by the design engineers.

\subsubsection{Overview of tunnel design procedures}

Mostly due to the particularity of each tunnel structure and the varying requirements and methods applying for differing clients (or sometimes even insurance companies), there is no explicit design code to apply for such structures. However, a rather comprehensive list 
of tasks and aspects that most usually need to be considered throughout a tunnel design is manifested below.

- Design Element Statement.

- Design stages; Design Checking

- Selection of Materials

- Structural Materials

- Miscellaneous Materials

- Interpretation of Geotechnical \& Hydrogeological Data

- Methodology

- Soft soil tunnels

- Rock tunnels

- Geological/Geotechnical identification

- Identification of faults and faulted zones

- Description of the soil /rock

- Soil parameters - derivation of geotechnical parameters

- Soil classification (USCS, AASHTO)

- Hoek-Marinos Geological Strength Index (GSI)

- Beniawsky RMR system

- Barton's Q-system

- Austrian Society for Geomechanics

- Derived Parameters

- Rock Tunnels

- Soft soil tunnels

- Geometry and tolerances

- Derivation of tunnel shapes

- Tolerances

- Methods of Analysis

- Geotechnical Geological conditions, Applicable Standards

- Analytical methods

- 2D Nummerical methods

- 3D Nummerical methods

○ Validation

- Excavation Support and stability 
- Classification of support systems (ASG)

- Contingency measures

○ Ground treatment / improvement

- Lining design philosophy

○ Primary Lining

○ Secondary Lining

- Design of Junctions (specific area of interest of the present study)

- Design of Headwalls

○ Multi-tunnel Design

- Waterproofing Design

- Ground movements and impacts

Design guidance for different aspects of tunnels may be found in respective publications of various scientific/professional organizations as for example the International Tunnel Association (ITA), the Austrian Society for Geomechanics (OEGG), the British Tunneling Society (BTS), the French Tunneling and Underground Space Association (AFTES). These design guides may as well be tailored to a project or a client, as for example the various Metro authorities (the London Underground Ltd, the Attiko Metro S.A. in Athens, the Wiener Linien / Wiener Stadtwerke Holding A.G. in Vienna, the Metro de Santiago S.A., the Metropolitan Transportation Authority - MTA of New York, etc.), or Crossrail with the socalled CEDS (Civil Engineering Design Standards).

\subsection{Tunnel junctions}

The formation of junctions in tunnels, e.g. at the locations of cross passages between twin-bored tunnels for emergency escape, or niches for equipment can be accommodated by the primary lining. The breakout for junctions is carried out from the main (larger) tunnel after the full tunnel profile has been constructed. The fully closed ring of support should be at sufficient distance ahead of the junction or niche (approximately 4-5 tunnel diameters) such that convergence of the main tunnel has stabilized prior to breakout. The design should assume the redistribution of stresses around an opening and potentially provide for appropriate pre-support at the locations of the junctions/niches which should be placed 
before an opening is realized. Pre-support can comprise local thickening of the sprayed concrete shell, additional reinforcement in the shell and rockbolts or dowels, according to the ground conditions.

The design of junctions may be based on empirical/analytical solutions (hole-in-plate models), or numerical solutions. For complicated situations, as e.g. large openings or multiple junctions at a location, it is expected that a three-dimensional numerical model can deliver a more reliable and rationalized design.

Beyond the structural issues that may appear at tunnel junctions, critical functional requirements may be compromised as well. These may be excessive differential deformations (which may also be handled within a numerical model) or waterproofing failure (for which the use of special systems such as re-injectable tubes to allow multiple treatments to halt leakage may be considered).

\begin{tabular}{|c|c|c|c|c|c|c|c|}
\hline Method & $\begin{array}{l}\text { Source/ } \\
\text { example }\end{array}$ & $\begin{array}{l}\text { Material } \\
\text { models }\end{array}$ & 2D or 3D & $\begin{array}{l}\text { Time } \\
\text { effects }\end{array}$ & $\begin{array}{l}\text { Ground water } \\
\text { effects }^{2}\end{array}$ & $\begin{array}{l}\text { Tunnel } \\
\text { shape }\end{array}$ & $\begin{array}{l}\text { Mined/ } \\
\text { TBM }\end{array}$ \\
\hline
\end{tabular}

Design methods for continua (i.e. 'soft ground' or massive rock):

\begin{tabular}{|c|c|c|c|c|c|c|c|}
\hline \multicolumn{8}{|c|}{ 'Closed-form' analytical methods } \\
\hline & $\begin{array}{l}\text { Muir Wood, } 1975 \\
\text { Curtis, } 1976 \\
\text { Einstein and } \\
\text { Schwartz, } 1979 \\
\text { Duddeck and } \\
\text { Erdman, } 1985\end{array}$ & $\begin{array}{l}\text { Elastic, } \\
\text { plastic, } \\
\text { creep }\end{array}$ & $2 \mathrm{D}$ & $\begin{array}{l}\text { Creep in } \\
\text { ground }\end{array}$ & Some & Circular & Both \\
\hline $\mathrm{CCM}$ & $\begin{array}{l}\text { Panet and } \\
\text { Guenot, } 1982\end{array}$ & $\begin{array}{l}\text { Elastic, } \\
\text { plastic, } \\
\text { creep }\end{array}$ & $2 \mathrm{D}$ axisym & $\begin{array}{l}\text { Creep, } \\
\text { timing of } \\
\text { support }\end{array}$ & No & Circular & Both \\
\hline $\begin{array}{l}\text { Bedded } \\
\text { beam spring }\end{array}$ & ITA, 1998 & Elastic & $2 \mathrm{D}$ & None & No & Any & Both \\
\hline $\begin{array}{l}\text { Stability } \\
\text { analyses }\end{array}$ & $\begin{array}{l}\text { Mair and } \\
\text { Taylor, } 1993\end{array}$ & Plastic & $2 \mathrm{D} / 3 \mathrm{D}$ & None & No & Circular & Both \\
\hline
\end{tabular}

Design methods for discontinua (i.e. jointed rock masses):

\begin{tabular}{|c|c|c|c|c|c|c|c|}
\hline $\begin{array}{l}\text { Stability } \\
\text { analyses }\end{array}$ & $\begin{array}{l}\text { Barrett and } \\
\text { McCreath, } 1995\end{array}$ & Plastic & $2 \mathrm{D}$ & Some & None & $\mathrm{N} / \mathrm{A}$ & Mined \\
\hline
\end{tabular}

Table 1: Overview of most common analytical approaches for tunnel analyses (adopted from [4]) 


\subsubsection{Theoretical approaches for openings in tunnels}

In general, the stress flow around openings (or more generically geometrical anomalies) can be well paralleled with flow mechanics for liquids. As seen in Figure 3 a simplistic but quite representative example is the flow of a stream in a free constant open riverbed, compared to an obstacle set within this flow; take for example a rock or a tree (a willow perhaps). This also implies that the shape of the obstacle (the breakout in the case of tunnel openings) can influence the stress distribution around it.

Concerning analytical solutions in general, several analytical methods have been developed in order to solve generic problems with applications to tunneling, or more focused tunneling problems. Typical problems are the stress distributions around holes in solid media, assuming a plane stress (plates) or plain strain (straight tunnels) situations, assuming elastic or non-elastic materials. An overview of analytical methods for tunnel analysis is given below as an excerpt from the BTS Tunnel Lining Design Guide [4] while typical examples of still used analytical solutions are included in [6], [9], [16], [31], [32]. Further discussion on analytical tunnel solutions is held in [23], where analytical models for openings/breakouts in tunnels are also discussed.

One of the most acknowledged solutions to identify stresses around openings in tunnels (a rather plane stress, or plane stress-strain problem) is the one provided by Kirsch for circular openings in infinite elastic plates (retrieved from [47]). This solution provides the stresses developed around an opening as shown in the Equations 1a-1c below and Figure 4 (Note: In all cases, $r \geq R_{i}$ and $\theta$ is taken CCW from the reference radius; $\lambda$ is the quotient of the vertical to horizontal stress in the plate). The calculation of the stresses is based on this solution and the consideration of the parent tunnel lining shell being developed (unwrapped) to a plane-stress state. Other analytical solutions, as for example those provided in [44] and [33], provide alternative correlations with the actual 2D plane stress. Roark's formulas [44] provide a good correlation with Kirsch for circular openings, while they also cover elliptical openings. Peterson [33] provides concentration factors for pipes under pressure with circular openings, but excluding data for bending around the opening. Herein the Kirsch solution is maintained as a reference for analytical solutions as it is a very common tool among tunnel engineers. 

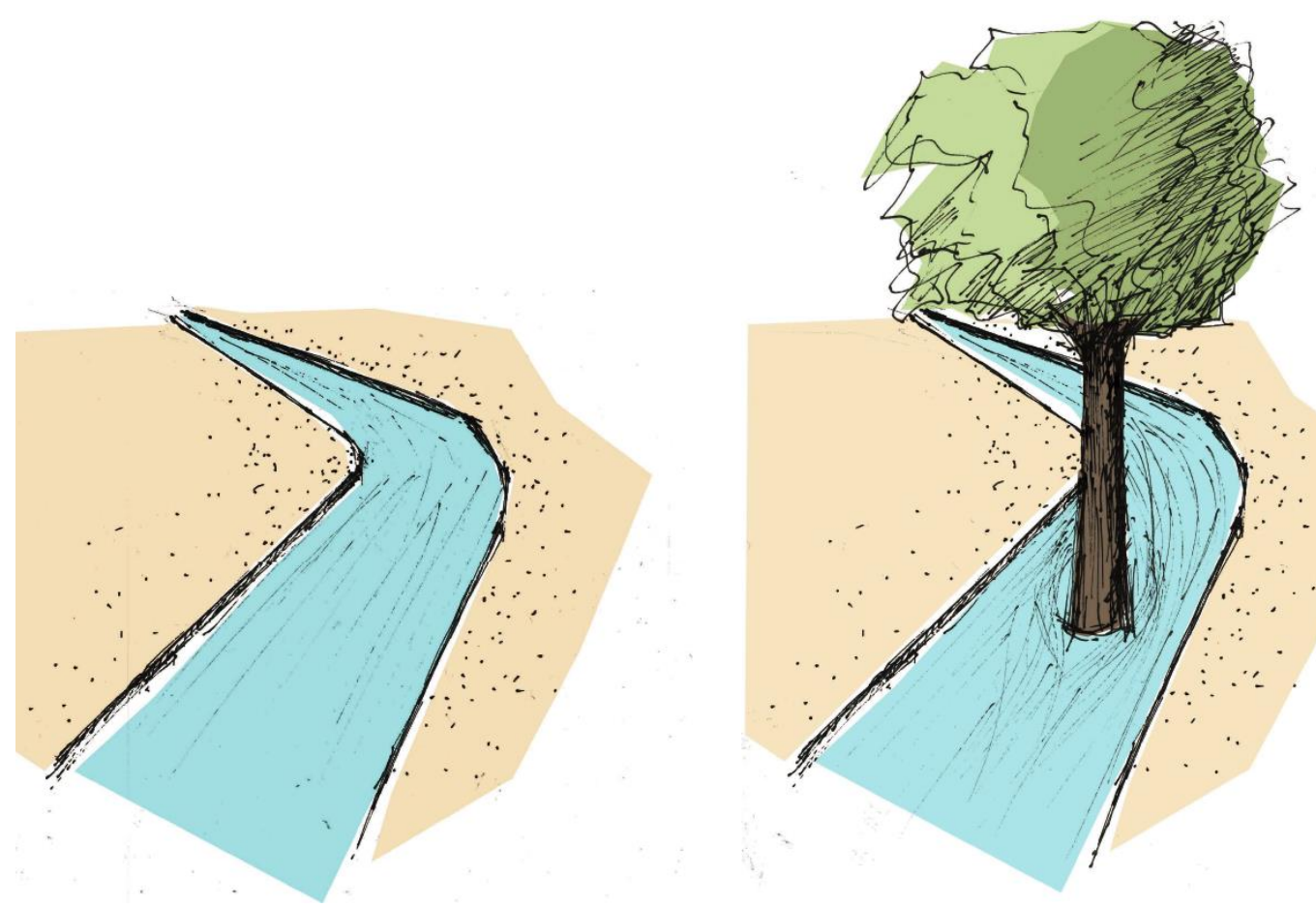

Figure 3: Natural flow model to represent flow redistribution around obstacles (thanks to Despoina Kalapoda of Foster \& P. for this sketch)

A strut-and-tie alternative solution [1] for openings in a two dimensional projection is graphically provided in Figure 5. In this approach, the compressive resultant is derived from the compressive hoop forces in the parent tunnel (see also Figure 6), and directed toward the vertical centreline of the opening. It is then split to two inclined compressive components (struts) either directed on the sides of the opening and a tensile component (tie) at the crown/invert of the opening which forms the force equilibrium above and below the openings. A compressive force is then delivered on either sides of the opening. Following the Kirsch theory (Equations 1a-1c) the tie should bear a force equal to the compressive force (hoop force - normalized per length unit) and the side struts carry a force of three times this force each at the hole's perimeter. The strut-and-tie model is shown symmetrical in the horizontal and vertical axis, but the proportions of the forces should be asymmetrical and subject to the opening geometry in case the opening does not have a circular (symmetrical) shape. In any case, the strut-and-tie model components are proportional to the original hoop force by a factor depending on the diameter of the parent tunnel and the size and shape of the child tunnel. 
Furthermore, as shown in Figure 6, assuming an arch model (pipe under uniform pressure), the average hoop force is virtually proportional to the average pressure acting on the structural element. In the case of shallow soft ground tunnels under pure geostatic conditions this pressure is derived by the soil's self-weight (depth $x$ unit weight); in the case of rock tunnels this pressure is defined by the rock's deformation/squeezing pattern.

In a three dimensional consideration of an opening on a tunnel lining, out-of-plane bending is anticipated. In this case, a complicated interaction of the forces in two in-plane coordinate directions of a curved shell emerges. Simplified, two cantilever and two arch support schemes may be identified as shown in Figure 7. The sections $A A$ and $B^{\prime} B^{\prime}$ resemble cantilevers supported by the body of the parent tunnel further beyond from the opening, therefore presented as spring-supported. The load acting on these cantilevers is reaction or pressure from the ground, and is therefore highly dependent on the confinement of the tunnel or the surrounding ground's stress state respectively; therefore they both may be assumed as proportional to the hoop force of the parent tunnel in the initial state, prior to breakout. Section BB may be understood as a situation between a fixed and a simply supported beam (hence spring-supported) with a uniform load, this being caused again by the overburden or generally the geostatic pressure. Section $A^{\prime} A^{\prime}$ can be idealized as a spring supported arch with both ends pushed toward each other due to hoop thrust with simultaneous elimination of the membrane behavior due to the opening; this thrust is delivered by the hoop force or its components described in the previous paragraph. In all cases a certain correlation between the bending moments around the opening and the hoop force (or the surrounding pressure) can be discerned. This relationship may be assumed by approximation as proportional.

$$
\begin{aligned}
& \sigma_{\mathrm{r}}=\frac{\sigma_{\text {vertical }}}{2}\left[(1+\lambda)\left(1-\frac{\mathrm{R}_{\mathrm{i}}^{2}}{\mathrm{r}^{2}}\right)-(1-\lambda)\left(1-\frac{4 \mathrm{R}_{\mathrm{i}}^{2}}{\mathrm{r}^{2}}+\frac{3 \mathrm{R}_{\mathrm{i}}^{4}}{\mathrm{r}^{4}}\right) \cos 2 \theta\right] \\
& \sigma_{\theta}=\frac{\sigma_{\text {vertical }}}{2}\left[(1+\lambda)\left(1+\frac{\mathrm{R}_{\mathrm{i}}^{2}}{\mathrm{r}^{2}}\right)+(1-\lambda)\left(1+\frac{3 \mathrm{R}_{\mathrm{i}}^{4}}{\mathrm{r}^{4}}\right) \cos 2 \theta\right] \\
& \sigma_{\mathrm{r} \theta}=\frac{\sigma_{\text {vertical }}}{2}(1-\lambda)\left(1+\frac{2 \mathrm{R}_{\mathrm{i}}^{2}}{\mathrm{r}^{2}}-\frac{3 \mathrm{R}_{\mathrm{i}}^{4}}{\mathrm{r}^{4}}\right) \sin 2 \theta
\end{aligned}
$$




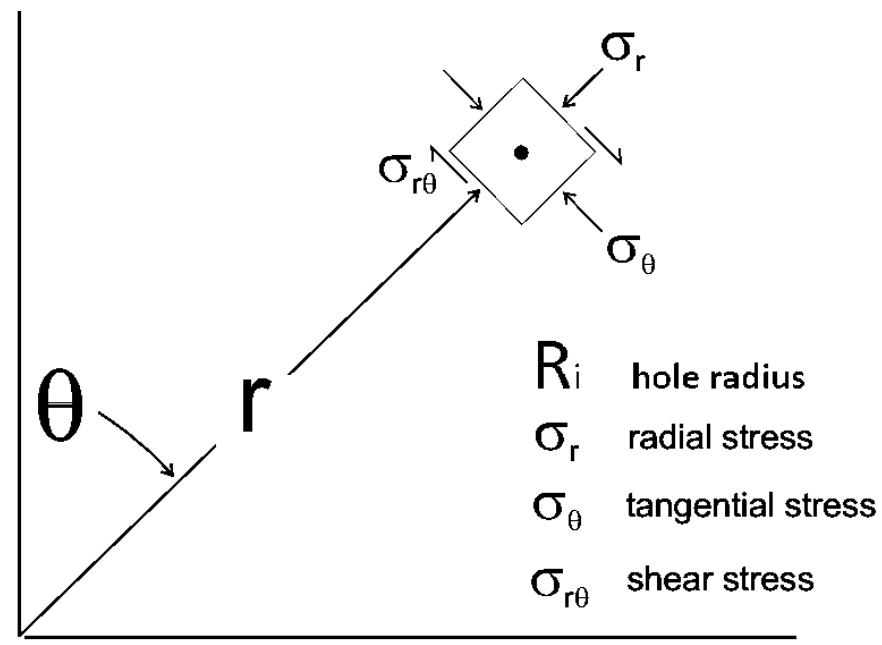

Figure 4: Notation of Kirsch formulas for stress assessment around circular openings

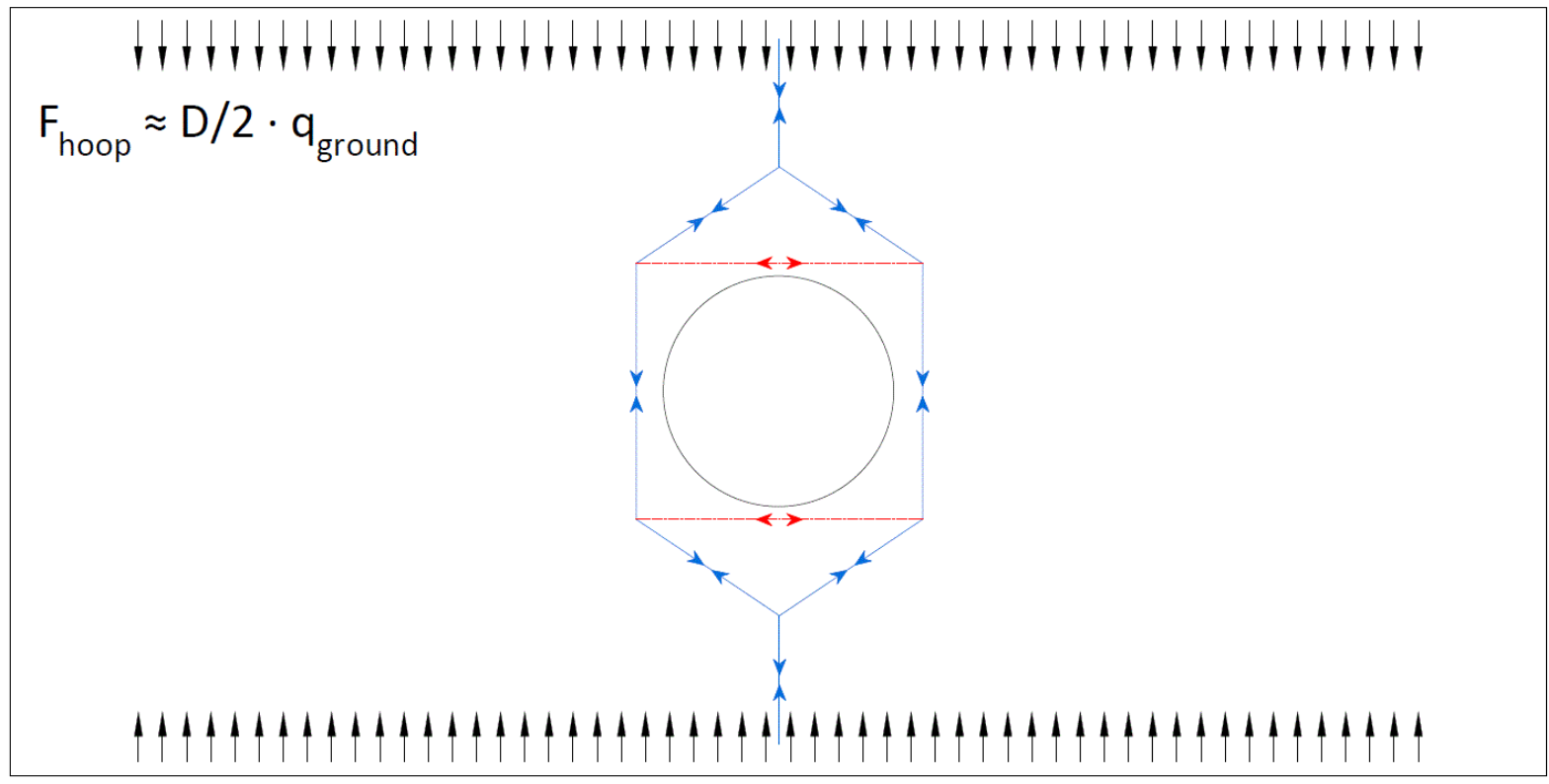

Figure 5: Strut-and-tie model for stress distribution around a circular opening in a plate 


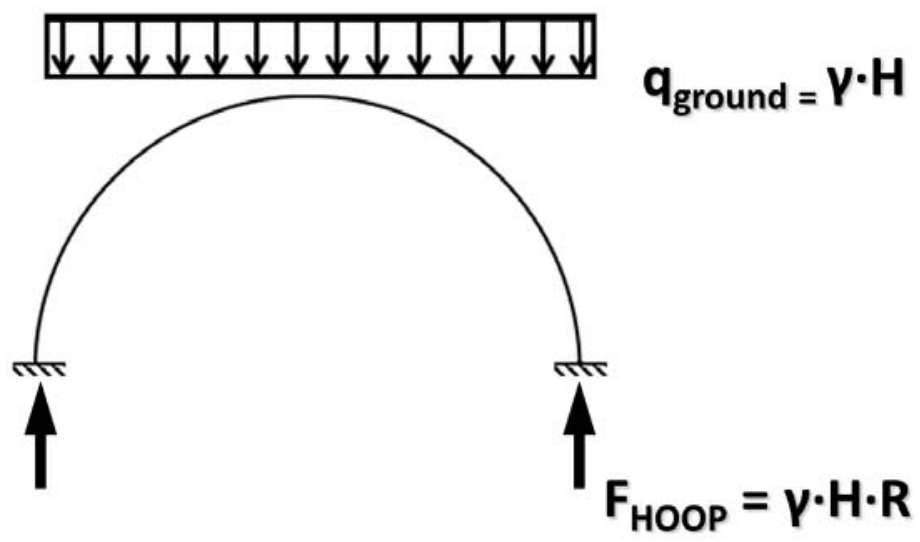

Figure 6: Arch model for a simplified 2D assessment of hoop forces in a circular tunnel
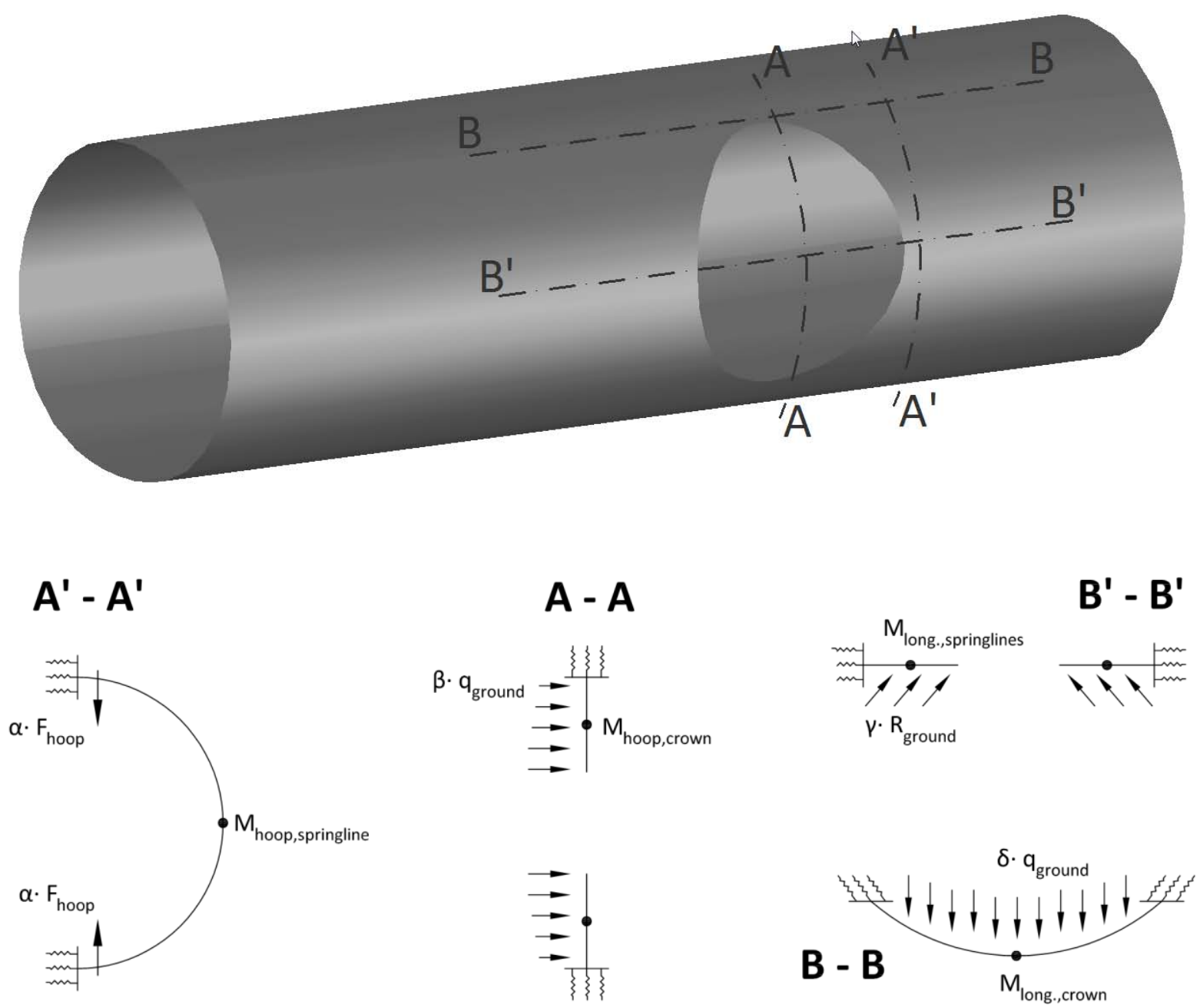

Figure 7: Simplification/idealization of static systems (down) responding to soil-structure interactions at characteristic sections (up) around a tunnel opening 


\subsubsection{Geometry aspects}

It should as well be highlighted that when it comes to tunnel junctions, circular shapes are hardly the case. Therefore the Kirsch solution may not be comprehensively applicable and accurate. This is due to the fact that

- Tunnel cross sections are very often non-circular, this being the rule when optimized (ovoid, or multi-radius) profiles are dictated by the design.

- The shape of the opening at a junction understandably (but sometimes misunderstood) differs from the cross-section of the child tunnel, i.e. the shape of the opening has the shape of the child cross section projected on the curved surface of the parent tunnel (see Figure 8 below)

- Moreover, the Kirsch solution considers infinite plates, this also not being the case in the investigated tunnel junctions.
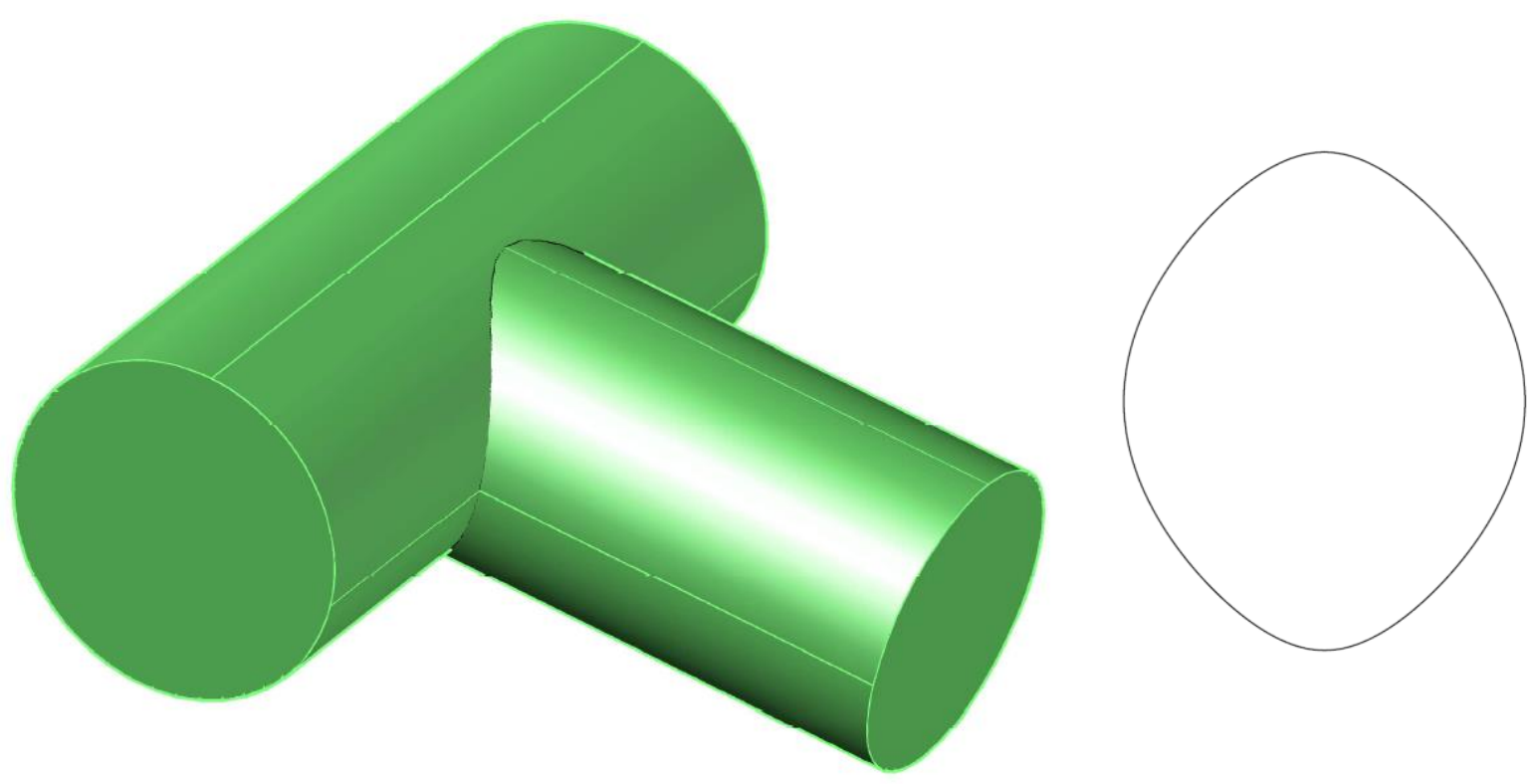

Figure 8: 3D Junction geometry (left) and actual junction projected/developed shape (right) for the case of two circular tunnels; aspect ratio is 0.9 
In the elementary case of circular tunnels and based on the geometry of the circular segment, the actual shape of the projected openings can be yielded based on the calculations in Figure 9, where:

d and D

S are the diameters of the child and parent tunnel respectively,

is the actual length of the opening at a section along the periphery of the parent tunnel (height of Figure 8 , right).

The left shape of Figure 9 is governed by Equations $2 a-2 c$, and the right shape is governed by Equation 3.

$\mathrm{r}=\mathrm{R} \cdot \cos \left(\frac{1}{2} \cdot \theta\right)=\frac{1}{2} \cdot \alpha \cdot \cot \left(\frac{1}{2} \cdot \theta\right)=\frac{1}{2} \cdot \sqrt{4 \cdot \mathrm{R}^{2}-\alpha^{2}}$

$\mathbf{s}=\mathbf{R} \cdot \boldsymbol{\theta}$

[Equations 2a-2c]

$\alpha=2 \cdot R \cdot \sin \left(\frac{1}{2} \cdot \theta\right)$

$\alpha=2 \cdot y=2 \cdot \sqrt{\frac{d^{2}}{4}-x^{2}}$

[Equation 3]
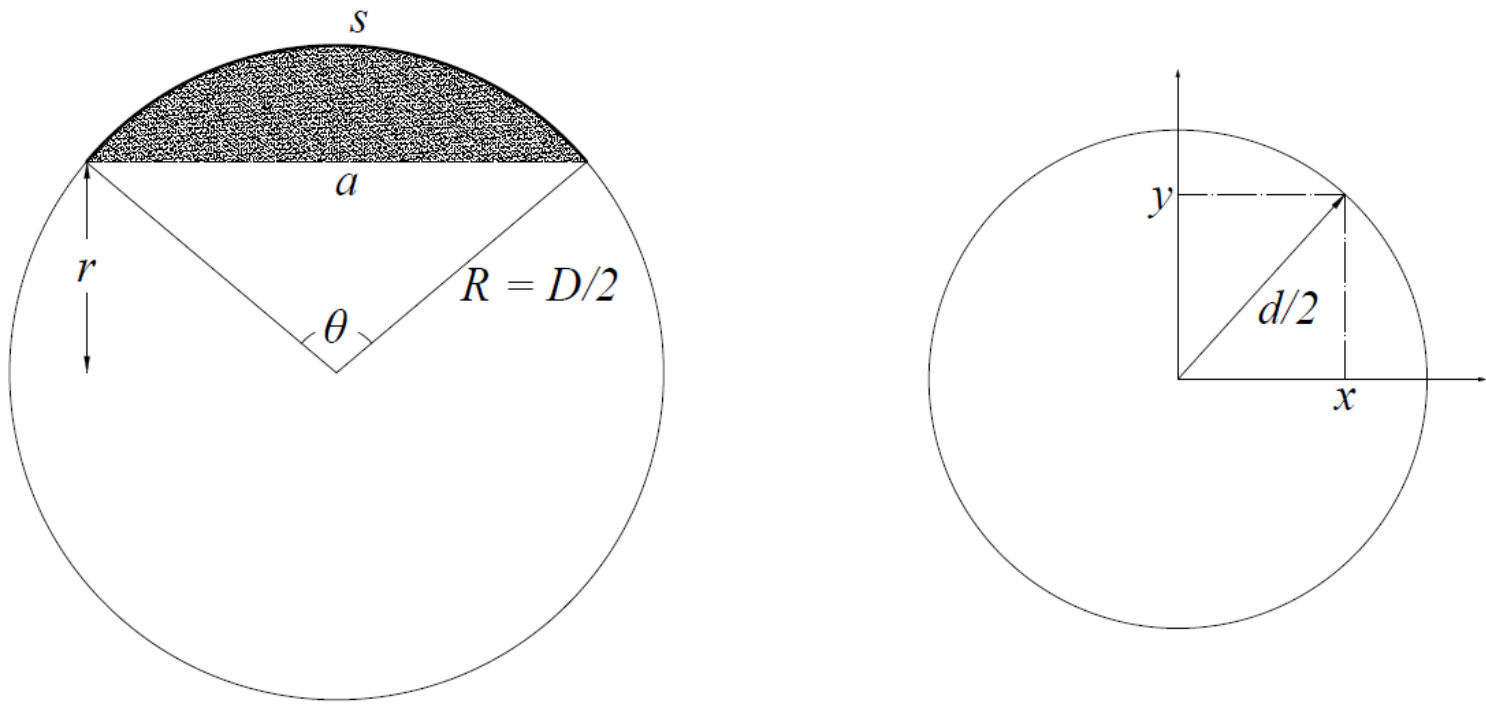

Figure 9: Geometry of the circular segment (cross section of the parent tunnel, left), and of the circular disk (cross section of child tunnel, right). 
Based on combination of the equations above, the actual coordinates of a developed (unwrapped) shape of the opening can be retrieved:

$$
\pm y= \pm \frac{\mathrm{D}}{2} \cdot \sin ^{-1}\left(\frac{2 \cdot \sqrt{\frac{\mathrm{d}^{2}}{4}-\mathrm{x}^{2}}}{\mathrm{D}}\right)
$$

This has been implemented for the setup of the 2D plate FE models discussed herein. For non-circular tunnel shapes this procedures may need much more complex analytical calculations [39] and CAD tools would be preferable.

\subsubsection{Numerical solutions for tunnel junctions / intersections}

As already seen, an advanced description of the structures geometry in the analysis procedure can allow for more realistic and arguably more efficient results in the design. Moreover, as the case is in most tunneling problems, significant soil-structure interaction phenomena take place, which can justify a more representative material modeling of both the soil and the support system. Consequently, numerical solutions that encompass a good description of materials and geometry are widely used for the analysis of junctions and intersections, while relevant case-history and research reports found in literature support this statement.

The work presented in [19] indicates the importance of three-dimensional modeling in the case of tunnel intersections, since it can provide additional information on deformations and plastic zones and consequently help to define the support measures at this location. Moreover [19] shows that the type and amount of support is dependent on the level of surrounding pressure (squeezing rock action) and that the proposed area of additional support around an intersection is also based on results of numerical analysis. In addition [18] reveals quantified areas of focus for the strengthening design based on both 3D numerical modelling and Artificial Neural Networks on a large number of analyses. The influence of the stress state on the tunnel intersection stress distribution and the intersection shape optimization is also argued in [35]. [40] discusses the deformation behavior of rock around a tunnel intersection with a 45 degree inclination through 3D analyses and showed that the influence area along the main tunnel was on the order of 1 to 3 diameters influence zone 
along the parent tunnel on the obtuse and acute direction with respect to the angle of the parent child tunnels. [30] concludes that large deviations emerged between two- and threedimensional models around intersections, while two-dimensional modeling overestimates the factor of safety near the investigated intersection.

A sophisticated 3D analysis is given for an actual case study in [20] which also reveals the potential of improved designs by use of 3D modeling at tunnel junctions. [43] highlights that provided the data collection, analysis and design procedure are carried out with diligence, sophisticated material laws for soil and shotcrete can facilitate an economic design of tunnel junctions. However the fact that reliable and valid calculations can be yielded through well selected simplifications is not excluded.

A broad discussion on the modeling approaches and the behavior of tunnel junctions is held in [22]. In this thesis, several further studies are reported, among them particular case studies with various findings regarding the behavior of tunnels at breakouts to junctions and intersections. In those it is certainly indicated that some correlations may be found between 2D and 3D approaches, yet not adequate to replace each other. Within this thesis, citing "Brown \& Hocking (1978): The use of the three dimensional boundary integral equation method for determining stresses at tunnel intersections" it is shown that the horizontal in situ stress coefficient may substantially influence the stresses at junctions; in particularly it is shown that higher horizontal stresses lead to lower stress concentrations in the tunnels. Furthermore, in the discussion of "Thareja et al. (1985): Three dimensional finite element analysis of branching tunnels", the stiffness of the surrounding medium (rock) is indicated as a decisive agent for the stress distributions around tunnel junctions. Based on background literature, as well as advanced numerical modeling compared with on-site measurements of a shaft-tunnel junction (at Heathrow terminal 4, i.e. in a soft ground shallow tunneling situation), [22] concludes that 3D modeling including ground and excavation sequences leads to the most reasonable prediction of stress distributions around tunnel openings, and the simpler analysis methods should give place to 3D numerical modeling whenever possible. [22] also highlights that the axial stress concentration was unaffected by stiffness, nonlinearity, anisotropy and plasticity of the ground, or its in situ stress distribution, yet the bending stress concentration was sensitive to stiffness of the ground and to a lesser extent the ground's yielding parameters (in the investigated case the undrained shear strength). In 
situ stress distribution was not validated as a significant influence for bending stress concentrations at a shaft-tunnel junction, yet a hypothesis was pronounced that this feature might be relevant for a tunnel-tunnel junction or a non-circular parent-tunnel.

\subsection{Numerical modeling and application in tunneling}

As computer technology has evolved in the recent years, numerical modeling appears to be an increasingly preferable solution in all engineering fields, including tunneling. However, with tunneling being one of the most empirical engineering disciplines, applicability and reliability of such numerical solutions may often be challenged. Simultaneously, the finite element method has become standard practice and is indeed a very useful tool for the analysis of complex tunneling structures (e.g. junctions already discussed in 2.2.3), as many recent design and consulting projects have shown.

Numerical analyses can virtually yield every result a conventional analysis can do, while they can capture complex geometries and realistic soil response mechanism and behavior, and they account for interaction between structures. A wide discussion on the advantages and disadvantages of numerical models is given in [34], while a critical evaluation on "desktop solutions" as opposed to empirical ones is also raised in [15]. Beyond any disadvantages of numerical analyses discussed among engineers, in the author's perspective a well prepared simulation of an underground structure can give a good and communicable description of the structural behavior, indicate risks, and highlight issues deserving additional attention during design or construction, providing substantial aid to the project development. Furthermore, the use of numerical solutions can as well facilitate to automatize the design (see also [16], [36]). This section discusses main aspects of modeling for tunnel analysis and design, with focus on the Finite Elements Method and SCL tunneling but without necessarily excluding applicability to other types of underground structures (e.g. segmental linings) or solution approaches (e.g. finite differences, or boundary elements).

\subsubsection{Implementation of numerical simulations in tunneling}

A typical procedure of building a tunnel numerical modeling includes 6 main steps: 
1. Pre-Modeling Decisions: This stage triggers an investigation of the modeling approach, mainly two dimensional (plane strain or axisymmetric), threedimensional (wished-in-place, or staged), or a combination of those using linear or non-linear models. Besides, in many cases it comes to question whether any adjacent existing structures should be included in the model. This is primarily driven by (a) the type of the project - i.e. research, design, design check, or back analysis - (b) the budget constraints, (c) the skills of the people involved.

2. Ground Modeling: This stage includes an engineered selection of the input parameters that has to be consistent with all input data from the field and previously acquired knowledge (e.g. literature, or previous projects in the area). At the same time the input parameters may need to be adopted in order to facilitate the particularities of the soil constitutive model used (e.g. specific strain-stiffening parameters, considerations for the groundwater seepage effects on soil properties, or ground improvement techniques). Potentially, anisotropy, heterogeneity, faulting, fissures are considered, and related uncertainties are managed.

3. Modeling of Structural Components: At this step, assumptions need to be made for what is relevant in case of support components in the model (e.g. should lattice girders or face support be taken into consideration) and which assumptions should be made for the design (e.g. preliminary dimensions, material properties of the lining, or even the effects of fiber reinforcement in the concrete lining).

4. Excavation \& Support Sequence: The excavation approach may need to be simulated in the model, since it can affect the soil-structure interactions and the requested results. When it comes to design of a tunnel, this is also an iterative procedure as the case is for the support.

5. Meshing (and boundary conditions): This comes to define the elements size/type, together with the discretization at areas of interest and where stress concentrations are expected. 
6. Trial analysis / Calibration: It is significant for the modeling procedure to identify faults and particularities in the model, through running a simple (e.g. linear elastic analysis, with coarse mesh, and simple elements) and only when the model behavior is transparent and explicable to calibrate the final optimized model. Calibration may be as well achieved on the basis of modeling with the same approach to a known project where monitoring data are available. A trial/calibration analysis should also pursue to investigate the effect of various parameters (sensitivity analysis) such as geometry of model, meshing/discretization, material parameters, etc.

The main aims of numerical modeling in the realm of tunnel engineering are to identify

- the ground behavior,

- the structural behavior of the support, and

- the environmental impacts of tunneling operations, that is mainly

(i) the settlements induced to adjacent structures and the surface, and

(ii) the disturbance of the groundwater.

A numerical model can prove to be very useful when the design needs to

- extract predictions from a coupled calculation, i.e. yield a certain set of displacements and section forces, the soil-lining interaction, or the interaction of settlements and the existing structures.

- accommodate complex ground behavior, or structural support response.

- accommodate non-linearity in the calculations (material, geometry, contacts)

- deal with non-regular tunnel geometries

Based on these insights, it is made easier to take decisions on various aspects on the design and construction procedures including the dimensioning of support elements, the 
type of complimentary support, risks - potential failure points, optimized excavation methods, and even to automatize design tasks.

Therefore, an advanced finite element analysis is selected herein in order to investigate the behavior of tunnel junctions.

\subsubsection{Material modeling - soil/rock}

Soils and rocks as natural materials are characterized by high irregularity, i.e. discontinuities, inhomogeneity, anisotropy and non-elasticity. Moreover, groundwater induces higher degrees of complexity. Depending on the severity of these features in a material with respect to the tunnel structural mechanics, and of course the limitations in modeling and computational effort, it may be reasonable to ignore some or all of the above mentioned irregularities in the substrate material. The simplest constitutive soil/rock model would then be continuous, homogeneous, isotropic, linear elastic.

Material nonlinearity in the model may be imposed by use of any of various constitutive laws, as for example the Mohr-Coulomb or modified Mohr-Coulomb, the Hoek-Brown, Drucker-Prager, original or modified Cam-Clay models. It is generally understood that the Mohr-Coulomb model is a most basic representation of plasticity in the realm of geotechnics. Beyond this model's weakness to describe certain critical state phenomena, it's benefits are that the model parameters are easily derived, and the results yielded by a Mohr-Coulomb model are easily interpreted and implemented. The criterion assumes that failure occurs when the shear stress at any point in a material reaches a value that is linearly dependent on the normal stress in the same plane; see Figure 10. Although a wide range of constitutive options is available, the present investigation is at an early simplified stage and the soil material is modeled as elastic isotropic (except for two trial models with Mohr Coulomb criteria for the soil material as discussed further below).

A further significant parameter associated to the ground is the in-situ lateral earth pressure coefficient, $K_{0}$, defined as the quotient of the pressure that soil exerts against a structure in the horizontal direction over the vertical one. 


\subsubsection{Material modeling - sprayed concrete linings}

Concrete linings in tunneling simulations may be modeled as linear elastic, or certain non-elastic constitutive laws may apply depending on the requirements of the analysis. In most cases non-linear analyses are implemented in order to identify expected deformations and crack widths in the concrete structure (this being one of the main serviceability criteria) or robustness/progressive collapse mechanisms. Non-linear concrete behavior has been subject to extensive research over the last decades and may be based on various constitutive models depending on the concrete behavior that needs to be better captured, examples are given below:

- Tension/Compression cut-offs

- Concrete Damaged Plasticity

- Discrete crack propagation model

- Smeared crack model (Fixed or rotating crack orientation)

Since tunnel analysis is usually handled through specialized geotechnical software, which may not contain sophisticated libraries with non-linear concrete constitutive models, a Mohr Coulomb formulation for concrete stands as an alternative. This can be applied and a good agreement with beam Timoshenko non-linear elastic lining elements and continuous MohrCoulomb elements has been indicated in [12] (see Table 2).

\begin{tabular}{|l|c|c|c|}
\hline \multicolumn{1}{|c|}{ Parameter set: } & $\begin{array}{c}\text { Primary Lining } \\
\text { continuum }\end{array}$ & $\begin{array}{c}\text { Secondary Lining } \\
\text { continuum }\end{array}$ & \multicolumn{1}{|c|}{ Units } \\
\hline Unit Weight & 25 & 25 & $\mathrm{kN} / \mathrm{m}^{3}$ \\
\hline Tensile Strength & 0.5 & 2.0 & $\mathrm{MPa}$ \\
\hline Cohesion & 1.5 & 1.5 & $\mathrm{MPa}$ \\
\hline Friction angle & 35 & 35 & $\mathrm{deg}$ \\
\hline Young's Modulus & 15 & 30 & $\mathrm{MPa}$ \\
\hline Poisson's ratio & 0.2 & 0.2 & - \\
\hline
\end{tabular}

Table 2: Continuum elements lining properties, corresponding to beam model properties of (i) Primary Lining: $E=15 \mathrm{GPa}, v=0.2, f_{c}=28 \mathrm{MPa}, f_{t}=0.5 \mathrm{MPa}$, (ii) Secondary Lining: $E=30 \mathrm{GPa}, v=0.2, f_{c}=28 \mathrm{MPa}$, $f_{t}=2.0 M P a ;$ adopted by $[12]$ 


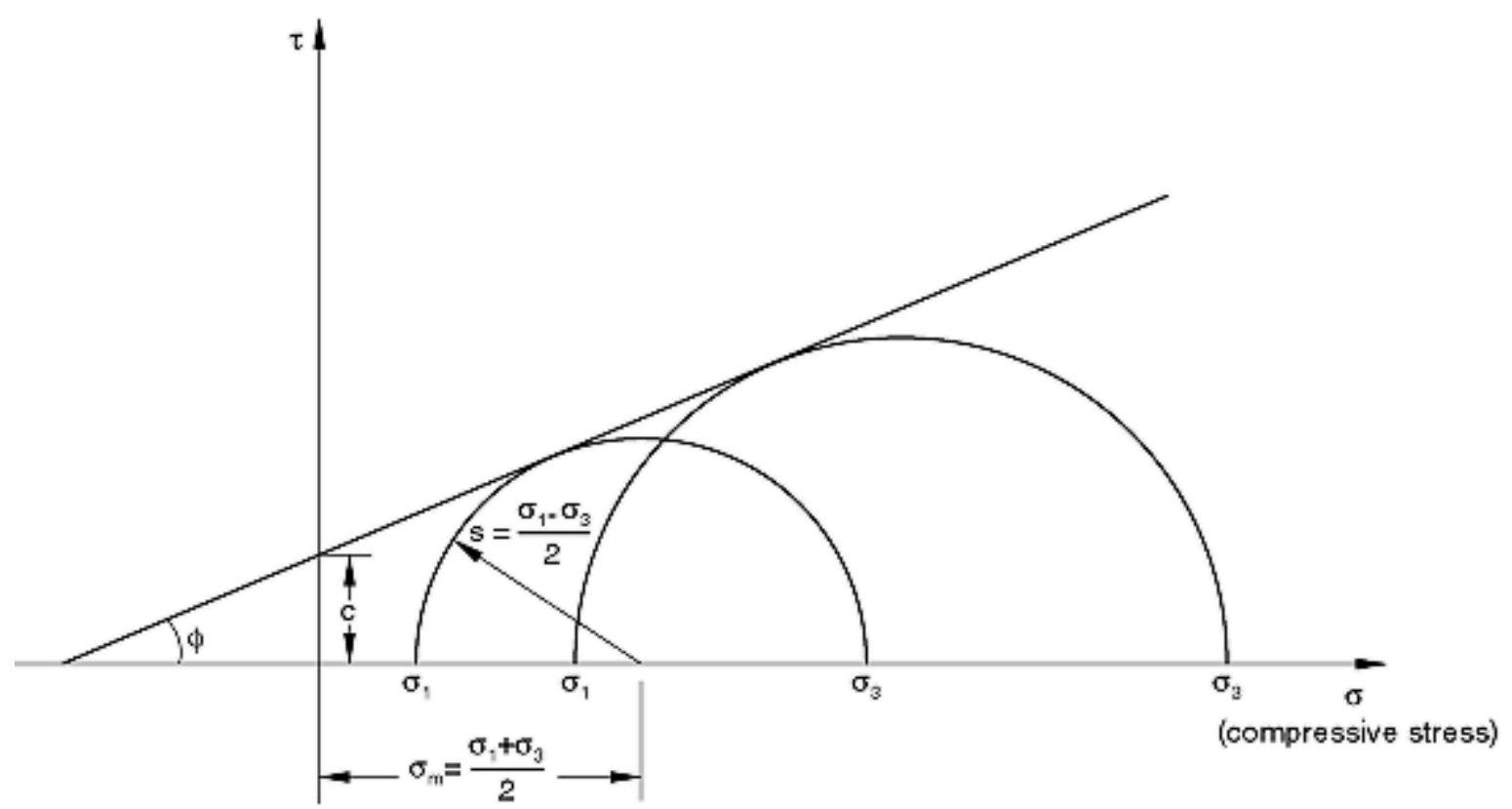

Figure 10: Graphical representation of Mohr - Coulomb failure criterion

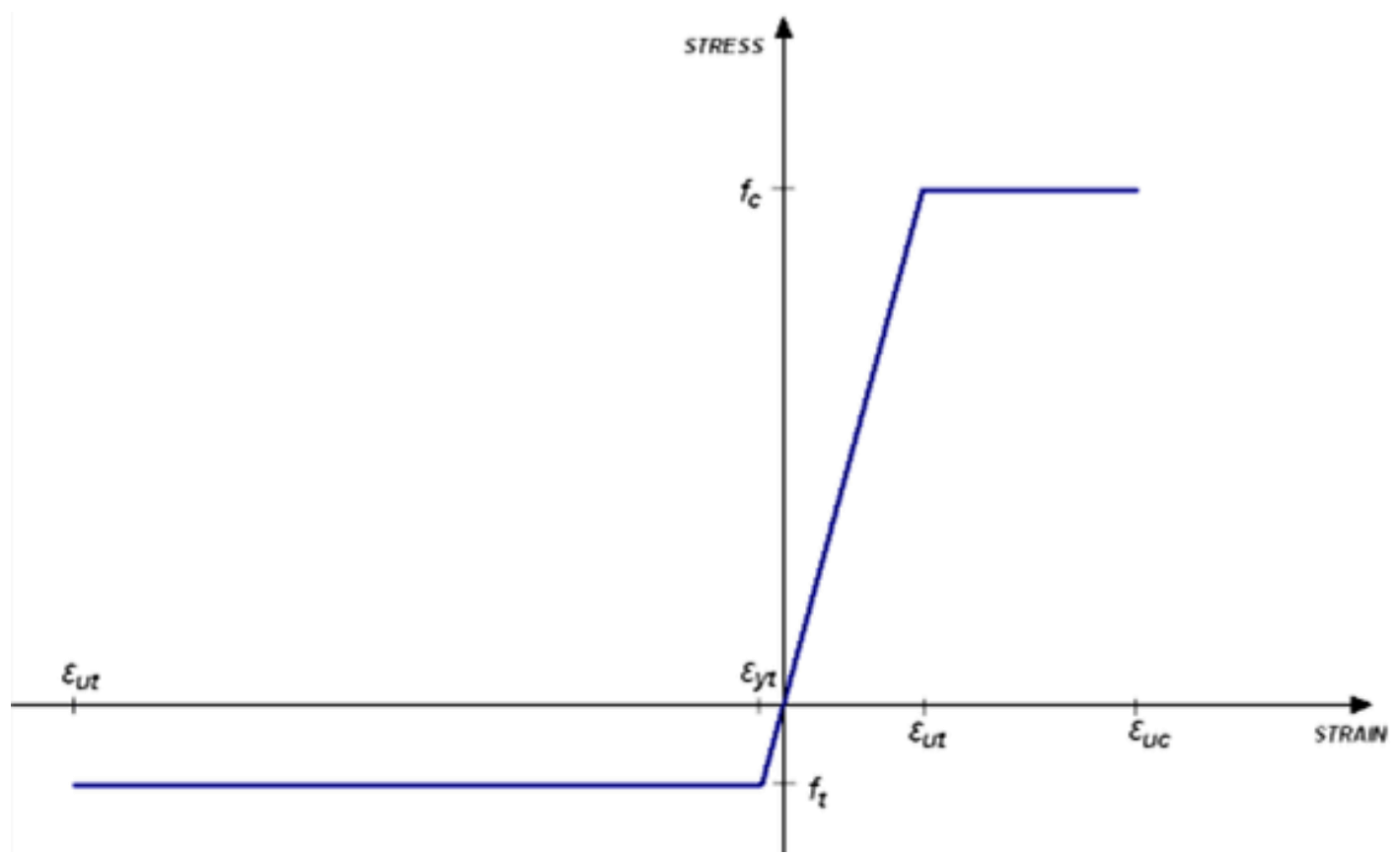

Figure 11: Graphical representation of linear-elastic perfectly-plastic concrete damaged plasticity material constitutive law 
Non-linear concrete models may be simulated using an appropriate one of the various constitutive laws available in literature and commercial analysis programs. Abaqus facilitates the use of the Concrete Damaged Plasticity model. The concrete damaged plasticity model allows for a good definition of the stress-strain relationship of concrete in both tension and compression. In that case the "concrete damaged plasticity" model represents linear plastic branches in both compression and tension sides defined additionally to the elastic material properties; see Figure 11. As seen, it assumes that the main two material failure mechanisms are tensile cracking and compressive crushing, while the crack widths may be implicitly calculated through the total lining strains multiplied by a crack spacing [11] [42]. A drawback of this particular constitutive model is that in order to define the material plasticity through the flow potential and the yield surface it requires input seldom readily available for the material used, i.e. concrete dilation angle (to account for the confinement effects), eccentricity of the flow potential function, ratio of the initial equibiaxial compressive yield stress to initial uniaxial compressive yield stress $f_{b 0} / f_{c 0}$, the ratio of the second stress invariant on the tensile to the compressive meridian $\mathrm{K}$, and the viscosity parameter that defines the viscous stiffness degradation after initial yield [12], [28], [26], [46]. Default values of these parameters acc. to [12] are given in Table 3, and a method for their derivation is also given in [21]. This constitutive model is not implemented herein but it shall be the first option within further investigations, when a non-linear concrete analysis is pursued.

\begin{tabular}{|l|c|}
\hline Dilation Angle & 35 \\
\hline Flow potential function eccentricity & 0.1 \\
\hline $\mathrm{f}_{\mathrm{b} 0} / \mathrm{f}_{\mathrm{c} 0}$ & 1.16 \\
\hline $\mathrm{K}$ ratio & 0.667 \\
\hline Viscosity Parameter & 0 \\
\hline
\end{tabular}

Table 3: Recommended/Default parameters for the concrete damaged plasticity constitutive model, according to [12] 


\subsubsection{Decision on modeling approaches}

Every model in the realm of civil engineering (as virtually in all natural sciences) is only a abstraction of reality (Figure 12). This can be due to the lack of knowledge, to randomness in the nature of materials and phenomena, to mathematical indetermination, even decisions on budget allocation; in the famous words of G.E.P. Box, "All models are wrong, some are useful" [3]. Consequently, the efficiency of a numerical model should be understood in the context of the amount and purpose of information needed to be extracted. At the same time it should be consistent with the limitations of the project, typically in terms of work-time or computational power available. Then it comes to the hands of the modeling engineer to prepare a tool that fits the needs of a problem with adequate accuracy, precision and reliability.

Calculation tools for engineers have vigorously evolved in the last few decades. For a long time before, the main calculation aid for a practicing engineer would be a slide rule around 1970 appeared what we nowadays know as a pocket calculator, but already in the early $80^{\prime}$ s personal computers would enter average design offices. Since then, PC technology is seeing a significant development rate allowing for increasing model complexity to be managed in reasonable budgets and computing time. Lately, advances relevant to structural numerical modeling are seen in the multi-core processors and Solid State Drives, while also powerful sets of D-RAM stay within reasonable costs. Yet, a remarkable shift is taking place when it comes to analyses in the world of civil engineers; as more sophisticated models are now technically feasible, the modeling work-time is shifting from the computational time per se to the time needed for building, troubleshooting, and post-processing the model by the user. Therefore, it is still put into question which modeling approach is to be selected for different problems, while standard approaches in tunneling are 2D plane strain or axisymmetric models, 3D wished-in-place approaches (see further description in 3.6), 3D staged approaches (simulating the excavation advance steps), or a combination of those on different parts comprising the studied tunneling project (take for example the detail of an underground station in Figure 13). Then, an efficient model should be envisaged as one solving the problem to which the decided resources are devoted, yet without yielding superfluous information. The effort and budget put in a modeling campaign needs to be aligned with the requirements of the project, i.e. the technical questions that need to be 
answered by the analysis in a feasible budget. In other words, a well-engineered numerical model should provide an appropriate balance between the output it provides, the effort needed to be managed, and the project specific added value it offers.

These concepts (also further discussed in [37]) have driven the final decision on the modeling campaign presented in the framework of this thesis, as described in the next section.

\section{ENVIRONMENT}
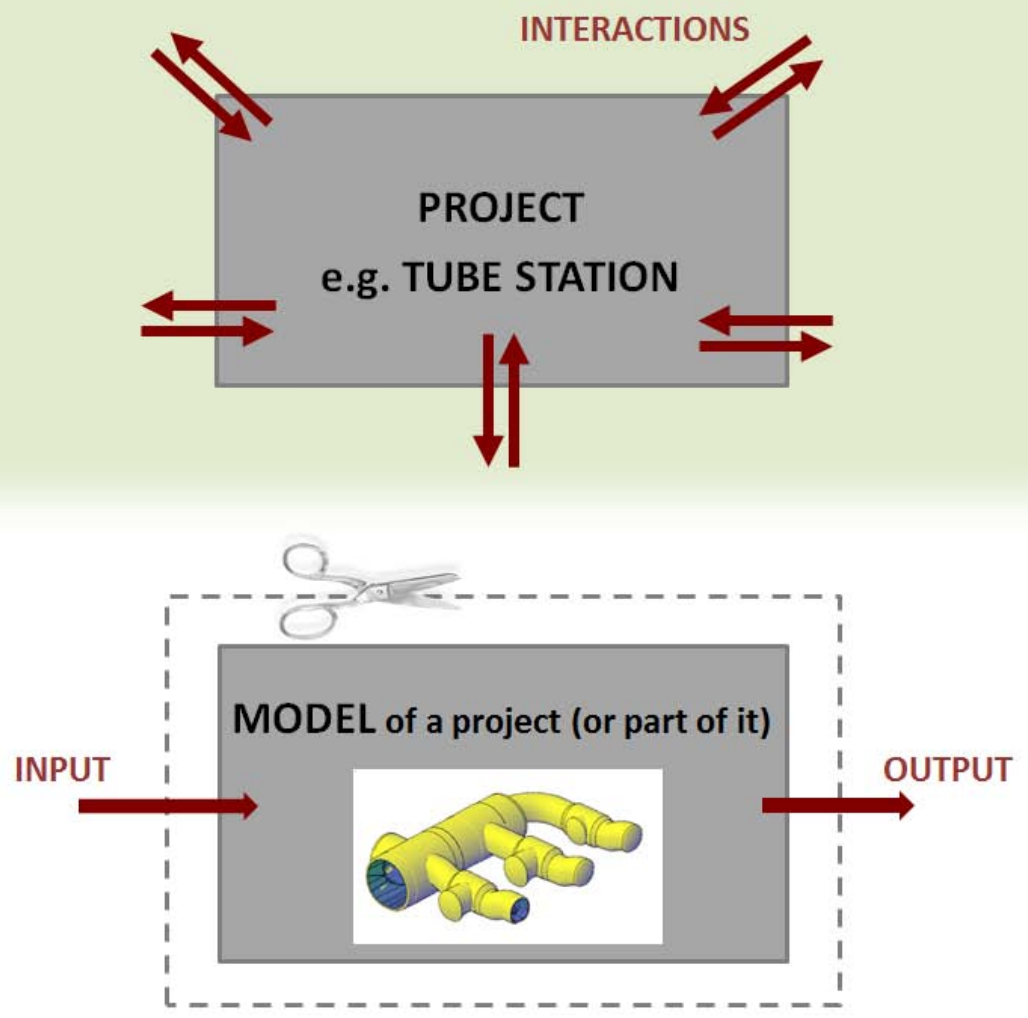

Figure 12: Illustrative description models as idealized abstractions of reality, neglecting certain aspects of the project's environment 


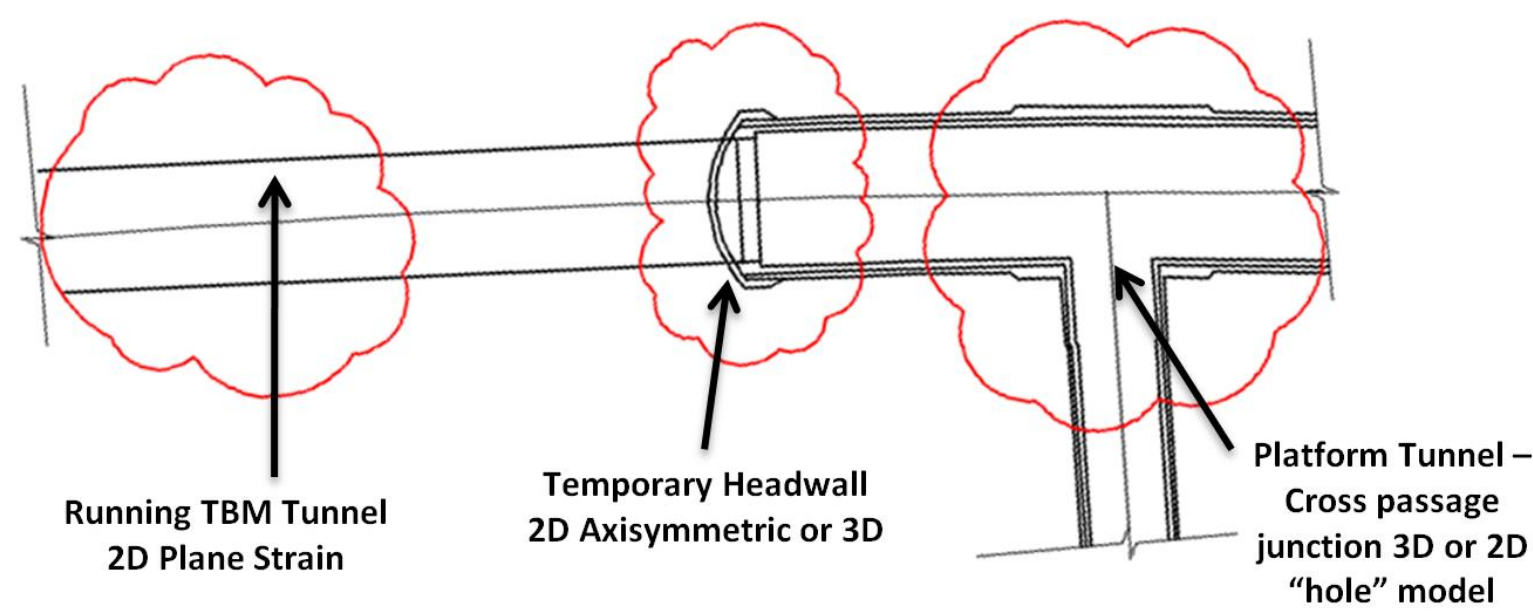

Figure 13: Typical examples of different approaches for numerical analyses depending on the structural element type 


\section{Investigation approach \& methodology}

\subsection{Basics}

In the present report, a sensitivity analysis is presented in order to disclose the impact of certain parameters to the structural behavior of junction openings. This investigation is based on numerical calculations by use of the finite element method, while some insights included in the studies are derived from the author's experience in design and consulting practice. The software used for the analyses is Abaqus CAE of Dassault Systems and some elements of this software are presented herein. Furthermore the investigation-specific details are presented, i.e. details of the modeling approach and the parameters assumed and implemented.

The analyses have been carried out mainly on 3D models, where the model utilizes 3D continuum elements in order to simulate the soil, and 3D shell elements to model the behavior of the lining. In order to investigate the relevance and adequacy of the 3D models and for the sake of a comparative representation, 2D plate models and Kirsch analyses have also been included in the investigations. For the latter, the load applied on the plate was set to $1 \mathrm{MN}(2.85 \mathrm{MPa})$ in the vertical direction to obtain a relationship of stress distributions before and after the opening. This is assumed to be effective and allow for extrapolation of the results for any initial stress state (i.e. any magnitude of hoop forces), on the basis of the superposition principle in elastic systems with small deformations.

\subsection{Objectives of the investigations}

As described in the previous section, a balanced selection between the modeling approach used in this research and the required results has been performed prior to conducting the studies. Within these studies, the aim remains to provide a comparative representation of various idealized structures, with respect to various geometrical and material properties. In particular there are two groups of models, i.e. (i) 2D plane stress models and (ii) 3D models. In the first case the delivered stress patterns are in-plane (no 
flexural response is accounted for), and the variation has to do with the aspect ratio of the two tunnels at junction (geometry). In the second case, three-dimensional modeling adds up to the information retrieved since flexural response of the lining is simulated, and the variation has to do both with the parent/child tunnel aspect ratio, and the soil's parameters (geometry and soil-structure interaction).

Finally, the models are engineered to deliver a comprehensive view of

- The effects of essential geometry and structural parameters.

- The consistency of analytical, two-, and three-dimensional methods.

- The stress redistribution around openings under various assumptions

The modeling effort in this case is the minimal needed to produce a set of results that give the response trend for variation of each investigated parameter ( 3 values for each parameter): Soil's modulus of elasticity, tunnel overburden, parent/child tunnel aspect ratio. In addition the effects of soil plasticity are checked for one model (one set of the above parameters). The renege of the investigated parameters is further discussed in 3.4.

\subsection{Finite elements software}

Abaqus - CAE (CAE stands for "Complete Abaqus Environment") is a software application combining all steps of the model, that being (a) the CAD formation of the model geometry, (b) the input of the modeling parameters, the mesh generator and generally the pre-processing, (c) the solver, and (d) the post-processing management of the results. Abaqus is a multipurpose program, and designed to serve complex analysis needs, by allowing to the user virtually full handling of the model, at basic level through a graphic user interface, or for special applications in Python programming language. In that sense, although not strictly a civil engineering tool, it provides a broad palette of modeling options. Considering (a) the fact that the constitutive material models used in the investigation that the present study consists part of were not expected to be particularly complicated, (b) the broad capabilities of the software, and (c) the availability of the program to the author, Abaqus was chosen as the most suitable program for the numerical analyses. 
Abaqus provides graphical and numerical output of different variables. Stresses, strains and displacements in the ground surrounding the tunnel structure are displayed using contour plots. The graphical output concerning the tunnel linings encompasses axial forces, bending moments and deformations. The application of Abaqus in tunneling has been demonstrated in the past, successful and verified closure of significant tunneling projects and research in the field has been documented and may be found in the references of this study [9], [25], [29], [38].

\subsection{Range of investigated parameters}

The model input comprises parameter sets for tunnel junctions that are expected to cause measurable opening and 3-D effects, i.e. concentration of stresses around the openings and moments respectively. Furthermore the selection of these parameters is envisaged to promote knowledge on the behavior of shallow tunnels under circumstances where the junction/breakout design becomes relevant. An overview of the investigated parameters is provided in Table 4.

The assumed substrate has a stiffness of 25-100 MPa in order to mimic soft ground, and a Poisson's ratio of 0.2 is applied overall. The density of the soil is assumed as $2000 \mathrm{~kg} / \mathrm{m}^{2}$ as a typical value for soil. The in situ earth pressure coefficient value is assumed $K_{0}=1$. Two exceptional cases with Mohr Coulomb plasticity have been run, where friction angle has been set to zero $\left(\phi=0^{\circ}\right)$, and cohesion has been assumed to be $50 \mathrm{kPa}$ and $100 \mathrm{kPa}$ respectively.

The geological model and the range of parameters used have been realistically selected based on an extensive survey of available geotechnical information on major recent $\mathrm{SCL}$ projects (see [5], [7], [8], [27], [41]) and have been juxtaposed to other relevant publications [2], [14], and they are anticipated to be representative of a typical tunnelling project in London Clay in Central London. Besides, the models use mostly elastic properties, and the selected values may also reflect several similar cases of shallow (urban) tunnels in soft ground. 


\begin{tabular}{|c|c|c|c|c|c|c|c|}
\hline $\mathrm{Sr} . \mathrm{Nr}$ & Type & $\begin{array}{c}\text { Poisson } \\
\text { Soil }\end{array}$ & $\begin{array}{c}\text { Youngsmod } \\
\text { ulus of Soil } \\
\text { [Mpa] }\end{array}$ & $\begin{array}{l}\text { Depth of } \\
\text { tunnel axis } \\
\text { in }[\mathrm{m}]\end{array}$ & $\begin{array}{l}\text { Depth - } \\
\text { Diameter } \\
\text { ratio }\end{array}$ & $\begin{array}{l}\text { Child - Parent } \\
\text { tunnel aspect } \\
\text { ratio }\end{array}$ & $\begin{array}{c}\text { Constitutive } \\
\text { model Soil }\end{array}$ \\
\hline & & $\mathbf{v}$ & Es & $\mathrm{H}$ & $H / D$ & $d / D$ & \\
\hline 1 & S.J.B. & 0.2 & 25 & 35 & 3.5 & 0.9 & elastic \\
\hline 2 & S.J.B. & 0.2 & 50 & 35 & 3.5 & 0.9 & elastic \\
\hline 3 & S.J.B. & 0.2 & 100 & 35 & 3.5 & 0.9 & elastic \\
\hline 4 & S.J.B. & 0.2 & 25 & 25 & 2.5 & 0.9 & elastic \\
\hline 5 & S.J.B. & 0.2 & 50 & 25 & 2.5 & 0.9 & elastic \\
\hline 6 & S.J.B. & 0.2 & 100 & 25 & 2.5 & 0.9 & elastic \\
\hline 7 & S.J.B. & 0.2 & 25 & 15 & 1.5 & 0.9 & elastic \\
\hline 8 & S.J.B. & 0.2 & 50 & 15 & 1.5 & 0.9 & elastic \\
\hline 9 & S.J.B. & 0.2 & 100 & 15 & 1.5 & 0.9 & elastic \\
\hline 10 & S.J.B. & \multicolumn{4}{|c|}{ Average normalized values from $3 D$ model } & 0.9 & elastic \\
\hline 11 & 2D-plstress & N.A. & N.A. & N.A. & N.A. & 0.9 & elastic \\
\hline 12 & 2D-Kirsch & N.A. & N.A. & N.A. & N.A. & 0.9 & elastic \\
\hline 13 & S.J.B. & 0.2 & 25 & 35 & 3.5 & 0.75 & elastic \\
\hline 14 & S.J.B. & 0.2 & 50 & 35 & 3.5 & 0.75 & elastic \\
\hline 15 & S.J.B. & 0.2 & 100 & 35 & 3.5 & 0.75 & elastic \\
\hline 16 & S.J.B. & 0.2 & 25 & 25 & 2.5 & 0.75 & elastic \\
\hline 17 & S.J.B. & 0.2 & 50 & 25 & 2.5 & 0.75 & elastic \\
\hline 18 & S.J.B. & 0.2 & 100 & 25 & 2.5 & 0.75 & elastic \\
\hline 19 & S.J.B. & 0.2 & 25 & 15 & 1.5 & 0.75 & elastic \\
\hline 20 & S.J.B. & 0.2 & 50 & 15 & 1.5 & 0.75 & elastic \\
\hline 21 & S.J.B. & 0.2 & 100 & 15 & 1.5 & 0.75 & elastic \\
\hline 22 & S.J.B. & \multicolumn{4}{|c|}{ Average normalized values from $3 D$ model } & 0.75 & elastic \\
\hline 23 & 2D-plstress & N.A. & N.A. & N.A. & N.A. & 0.75 & elastic \\
\hline 24 & 2D-Kirsch & N.A. & N.A. & N.A. & N.A. & 0.75 & elastic \\
\hline 25 & S.J.B. & 0.2 & 25 & 35 & 3.5 & 0.6 & elastic \\
\hline 26 & S.J.B. & 0.2 & 50 & 35 & 3.5 & 0.6 & elastic \\
\hline 27 & S.J.B. & 0.2 & 100 & 35 & 3.5 & 0.6 & elastic \\
\hline 28 & S.J.B. & 0.2 & 25 & 25 & 2.5 & 0.6 & elastic \\
\hline 29 & S.J.B. & 0.2 & 50 & 25 & 2.5 & 0.6 & elastic \\
\hline 30 & S.J.B. & 0.2 & 100 & 25 & 2.5 & 0.6 & elastic \\
\hline 31 & S.J.B. & 0.2 & 25 & 15 & 1.5 & 0.6 & elastic \\
\hline 32 & S.J.B. & 0.2 & 50 & 15 & 1.5 & 0.6 & elastic \\
\hline 33 & S.J.B. & 0.2 & 100 & 15 & 1.5 & 0.6 & elastic \\
\hline 34 & S.J.B. & \multicolumn{4}{|c|}{ Average normalized values from $3 D$ model } & 0.6 & elastic \\
\hline 35 & 2D-plstress & N.A. & N.A. & N.A. & N.A. & 0.6 & elastic \\
\hline 36 & 2D-Kirsch & N.A. & N.A. & N.A. & N.A. & 0.6 & elastic \\
\hline 37 & S.J.B. & 0.2 & 50 & 35 & 3.5 & 0.9 & $M C: \phi=0, c^{\prime}=50 \mathrm{kPa}$ \\
\hline 38 & S.J.B. & 0.2 & 50 & 35 & 3.5 & 0.9 & MC: $\phi=0, c^{\prime}=100 \mathrm{kPa}$ \\
\hline
\end{tabular}

Table 4. Analyzed parameters (S.J.B. stands for Single Junction Breakout, i.e. only one child tunnel). Lines 10, 22, 34 correspond to the averaged values from the 3D models for aspect rations $d / D$ of $0.9,0.75$, and 0.60 respectively. The material properties of concrete are elastic, with Young's modulus $E_{c}=15$ GPa and Poisson's ratio $\mathbf{v}_{\mathrm{c}}=\mathbf{0 . 2}$. Also see notation in Figure 14 . 


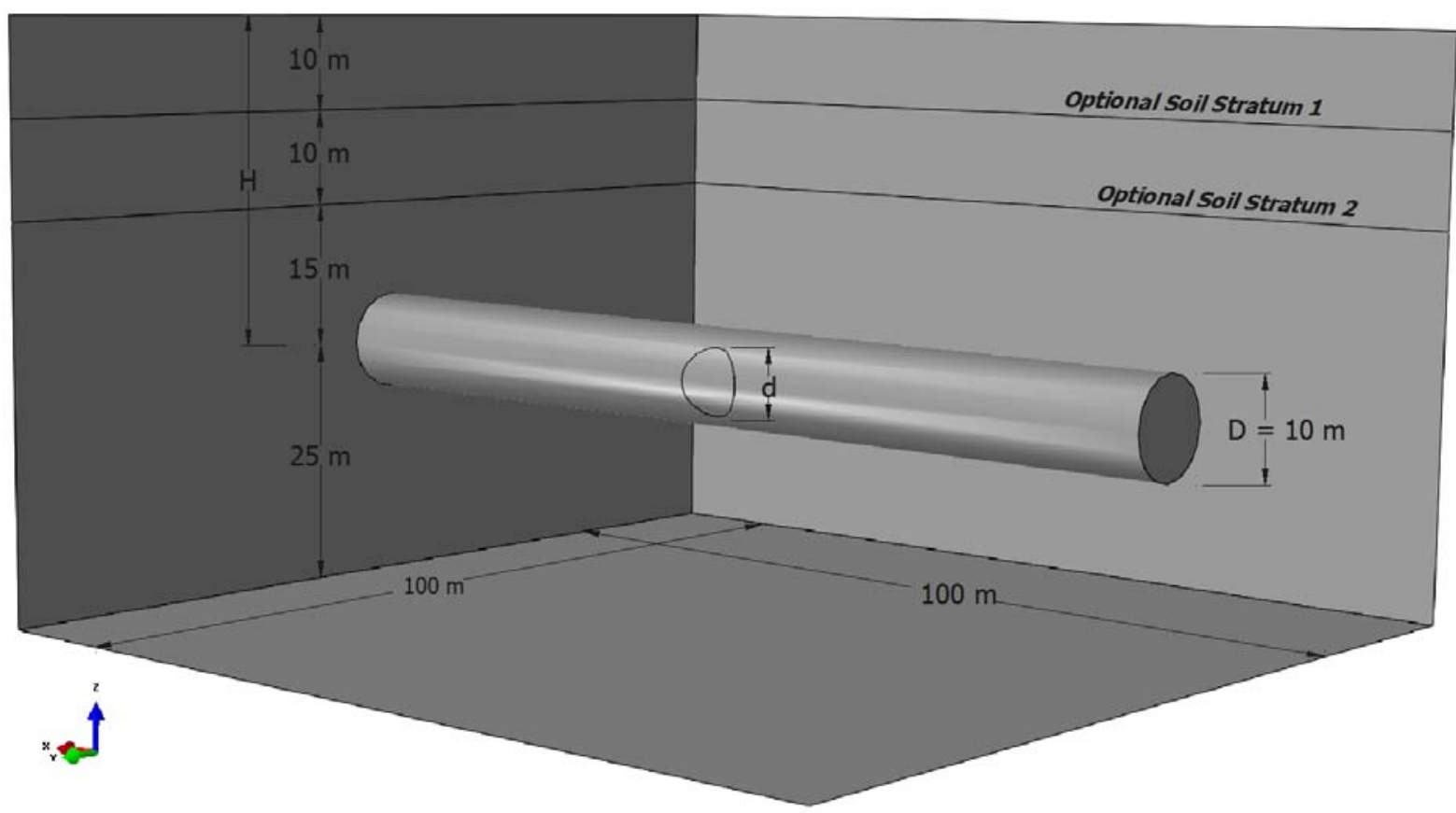

Figure 14: Definition of 3D modeling geometric parameters and notation (d: child Tunnel diameter, D: parent tunnel diameter, $\mathrm{H}$ : tunnel centerline depth)

Concrete's Poisson's ratio is set to 0.2. Although concrete stiffness is typically assumed in design to be in the order of $30 \mathrm{GPa}$, in these analyses it is set to $15 \mathrm{GPa}$ in order to roughly capture the time-dependent soil-structure interaction effects, that is the gradual bearing of concrete lining against the soil induced loads at an age shortly after full ring closure and below its full strength development ( 28 days as a standard). The lining thickness is set to 350 $\mathrm{mm}$ as a design value for normal concrete strength capacity against the hoop forces in a section of the discussed structures.

The tunnel is assumed to have a diameter of 10 meters, which may represent exemplarily a typical value for platform tunnels at underground rail stations. The depth of the structure is assumed 1,2 , or 3 diameters from the tunnel crown. The ratio of child over parent tunnel diameter size is selected to vary between $0.6,0.75$, and 0.9 , leading to breakouts to child tunnels with a diameter of $6 \mathrm{~m}, 7.5 \mathrm{~m}$, and $9 \mathrm{~m}$ respectively. 


\subsection{Modeling approach - 2D models}

The 2D model represents the developed (unwrapped) parent tunnel lining where an opening of the size of the child tunnel is performed. In these models also the non-circular shape of the breakout development has been considered, that is an elongated shape has been assumed for the opening.

The model geometry of the 2D models is shown for the case of model [2D-090] in Figure 15. The model of the developed parent tunnel is an in-plane loaded plate with a height of $31.4 \mathrm{~m}$ (the circumference of the parent tunnel) and a width of $90 \mathrm{~m}$, i.e. with more than 4 diameters distance between the side-boundaries and the focused region around the opening.

Indicative areas in the focused region around the opening, particularly at the crown, invert and spring-lines of the opening have been defined in the model (see also Figure 16). The results for each of the representative locations are taken from the respective geometrically defined frames at the lining. These frames have a size of 2 by 2 meters and are located at a distance of $350 \mathrm{~mm}$ from the breakout edge. This distance is provided in order to filter out high stress concentrations from the results which may appear due to numerical limitations. It is assumed that these high stress values are less representative for a design as they appear in a very small distance from the edge, smaller than the thickness of the lining itself. It is reminded anyway that this study aims to extract results for comparisons with each other than to extract design-relevant section forces.

The model is divided into sets of geometrical definitions, these being the lining, the breakout, and the above mentioned framed focus areas around the opening. These sets are specific features of the Abaqus and they are very useful in handling the management of model information, the property assignments, and the model development and running. These sets and their functions are similar to what is referred to as macro-elements in other FE software packages.

At this stage of the investigations, the concrete material used in these $2 \mathrm{D}$ analyses are governed by a simple isotropic linear elastic constitutive law with the properties defined in 
3.4, i.e. $15 \mathrm{GPa}$ and 0.2 for the Young's modulus and the Poisson's ratio respectively. The section assumes a $350 \mathrm{~mm}$ thick shell.

For the meshing of the model (Figure 16), a global element size of $1.25 \mathrm{~m}$ was applied, yet the focus region around the openings used a smaller element size of approximately $0.35 \mathrm{~m}$ (equal to shell thickness). The elements used were triangular and quadrilateral linear shell elements with finite membrane strains, specified as S4(R) and S3 in the Abaqus element library. For each 2D model the mesh comprised approximately 10000 elements.

The force applied in-plane on the top and bottom of the plate (Figure 17) is theoretically equal to the average hoop force of the respective parent tunnel. In the particular cases the force was set to $1000 \mathrm{kN} / \mathrm{m}$. Since the forces applied were counterbalanced there was no need for boundary condition definitions, i.e. the model was free of any supports.

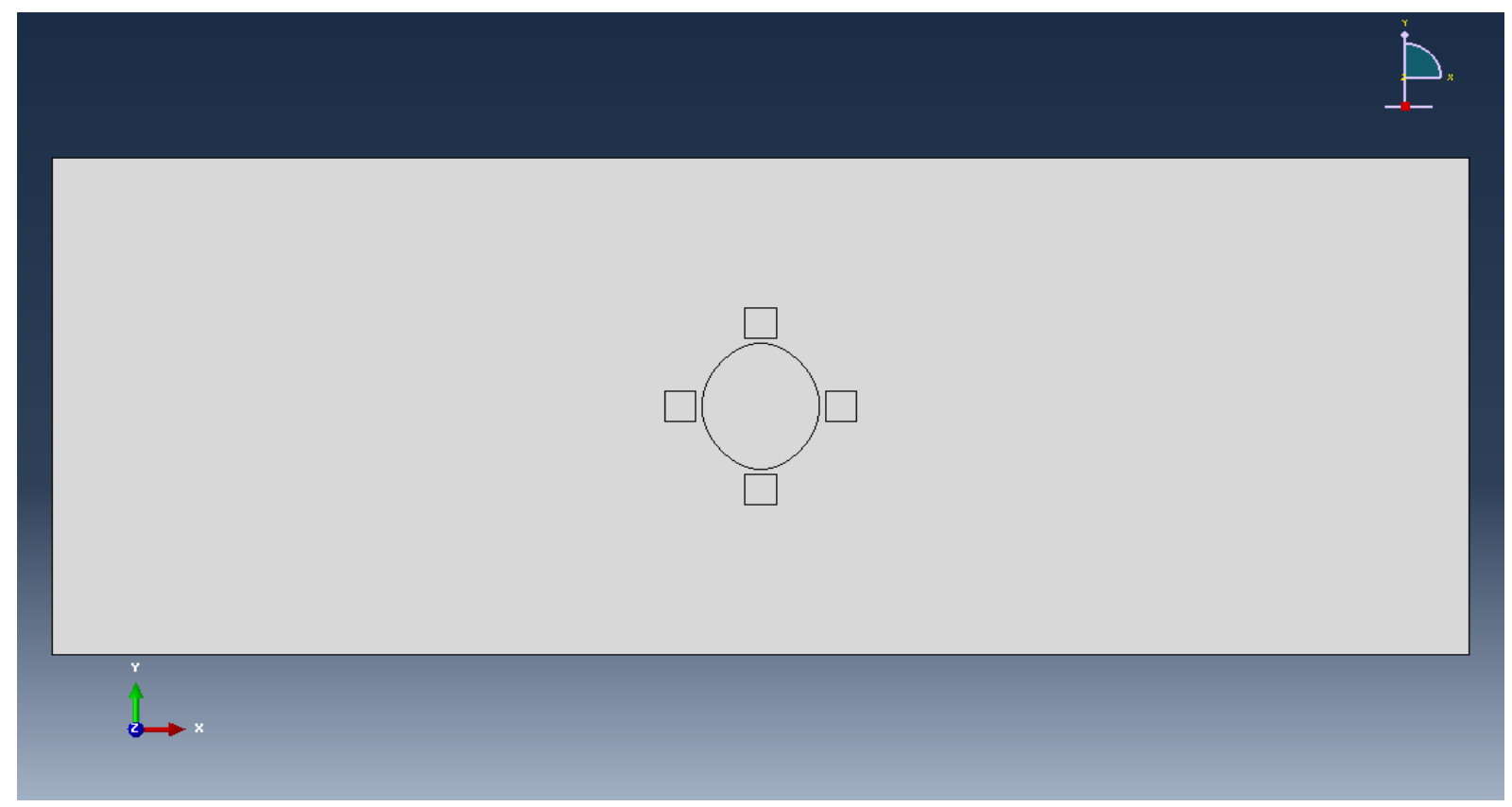

Figure 15: Indicative geometry of a 2D plate model (for child/parent tunnel diameter $=0.9$ )

Two steps were programmed in the model, these being

1. the initial loading condition in the complete plate by application of the above mentioned in-plane force, and 
2. the removal of the breakout which generated the stress redistribution around the opening.

This function was manually defined by editing the input text file of each analyzed model.

The output of the model was calibrated to produce only purpose-relevant values, these being mainly deformations and elastic strains, stresses, and section axial forces in both directions in the plane of the shell.

The analysis was based on a standard Newton-Raphson algorithm, with the default Abaqus tolerance and iteration values, which are assumed adequately precise for the discussed civil engineering problems [12].

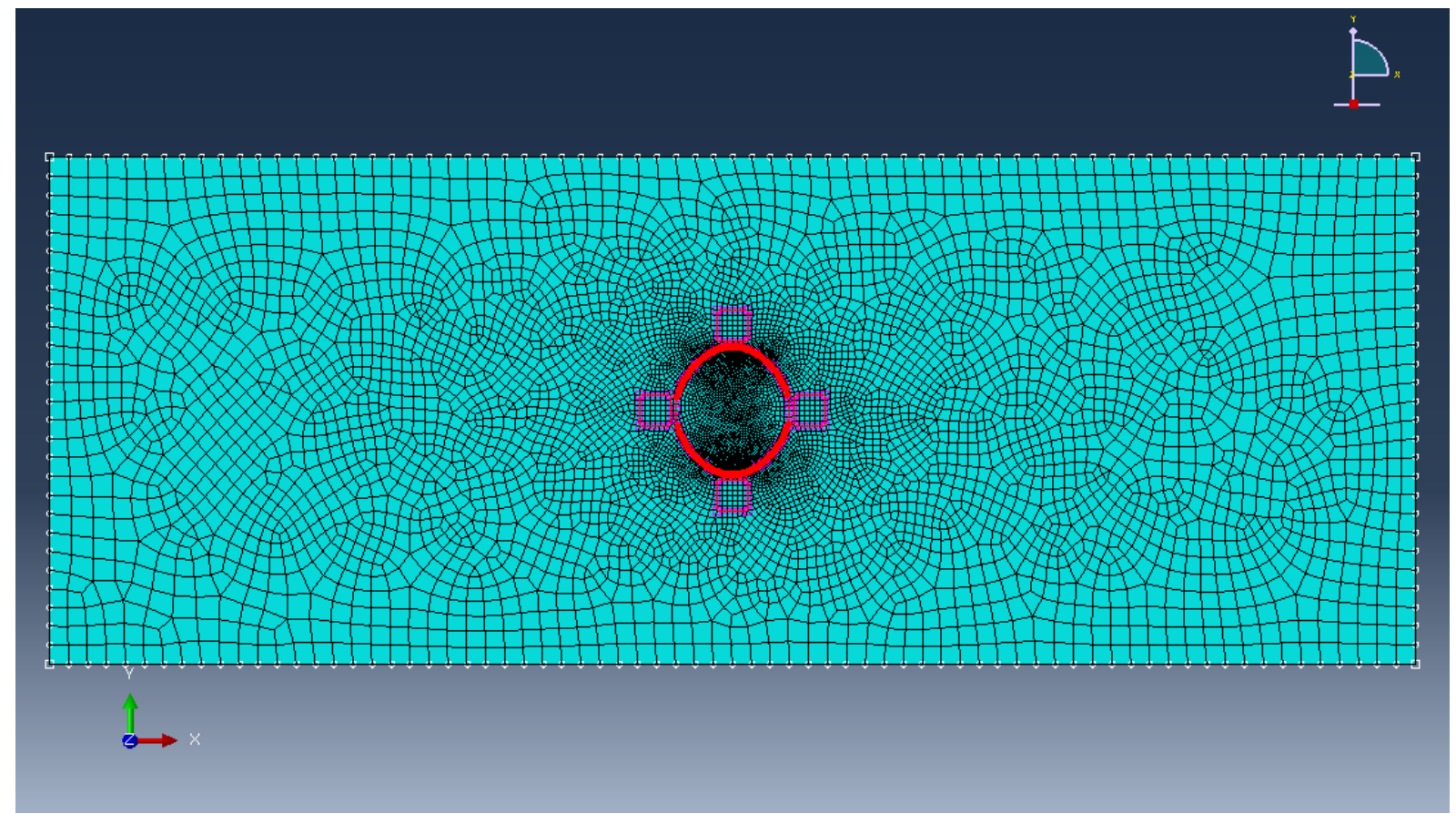

Figure 16: Indicative mesh of a 2D plate model; highlighted lines indicate regions with local increased mesh density 


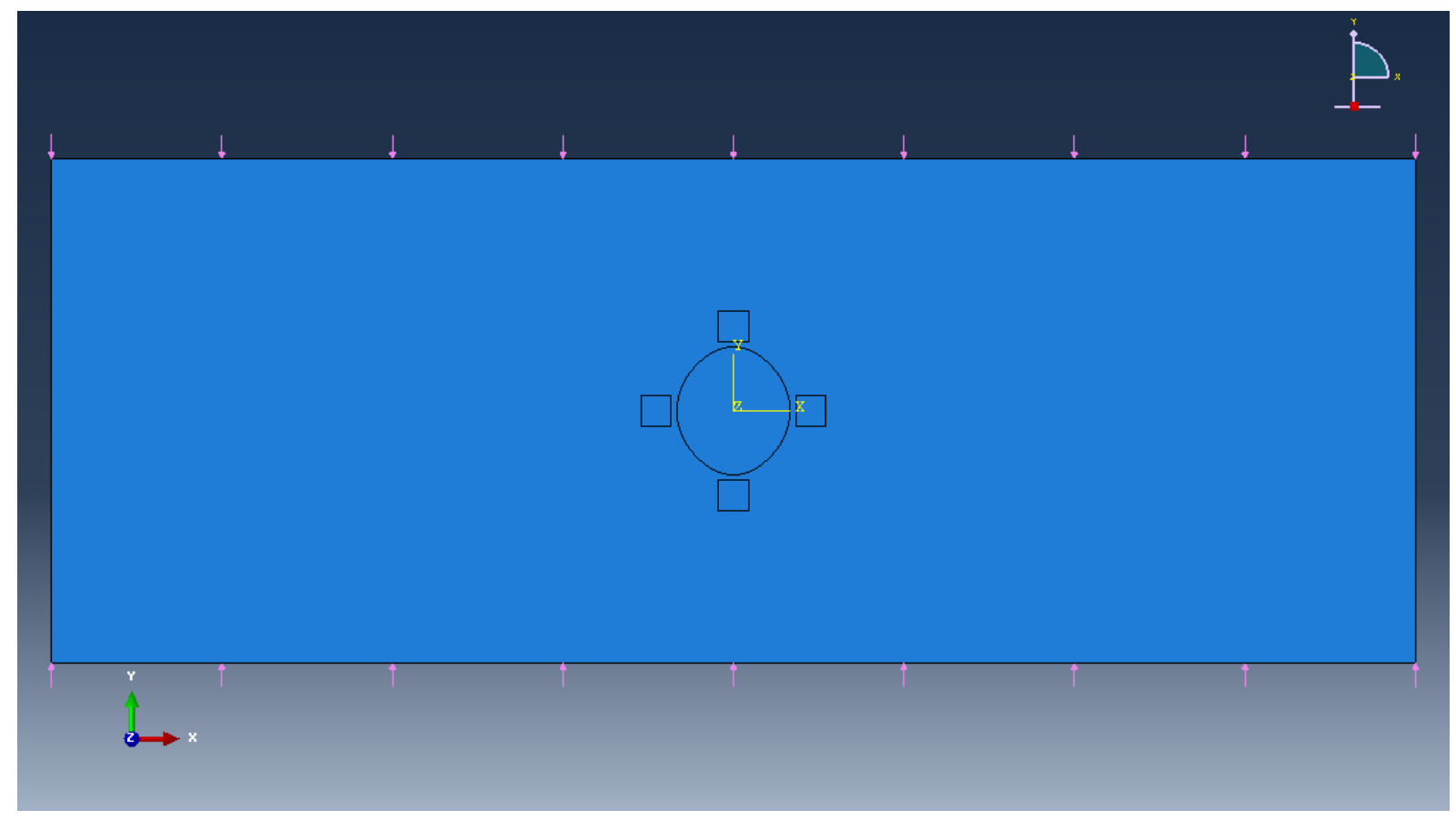

Figure 17: Load application for a 2D plate model

\subsection{Modeling approach - 3D models}

The 3D model captures the stress distribution of the tunnel breakout in space, allowing for the retrieval of moment and shear values in the shell of the tunnel lining after the breakout and potentially a more realistic representation of the stresses and deformed shapes compared to the 2D plate models described above.

The geometry of the 3D models is shown for the case of model [3D-25-35-090] in Figure 18, Figure 19, and Figure 20. The sizes of the soil block (Figure 18) are 100, 100, and 40 to 60 meters in the $x, y$, and $z$ axes respectively. The soil model extends to $20 \mathrm{~m}$ (i.e. 2 diameters) below the invert of the tunnel; setting this boundary at this location is anticipated to provide for generally realistic effects regarding primarily the effects of the rigid boundary to the stress field around the opening, and secondarily the unloading effect of the elastic model at excavation (removal) of the tunnel core. In Figure 19, the tunnel lining is highlighted in red. The length of the tunnel itself is $90 \mathrm{~m}$ and as illustrated it does not extend to the boundaries of the soil leaving a soil material of $5 \mathrm{~m}$ thickness on either side. This aimed to minimize the boundary effects on the tunnel tube under radial compression, i.e. the longitudinal compression that would develop for such a load situation, for constrained ends, and for a 
tube's material with Poisson's ratio other than zero. The longitudinal strains are then absorbed by these $5 \mathrm{~m}$ thick "soil-pillows" once the excavation is simulated and the tunnel is loaded. It needs to be noted that such a longitudinal restraint is not necessarily excluded in an actual tunnel, yet the compression effects generate a multi-dimensional compression in the lining material and thus the FE results are expected to be less onerous. As further illustrated, the geometry of the soil bulk is divided in the top two layers, each of $10 \mathrm{~m}$ thickness to facilitate the modeling procedure for the various different overburdens presented in 3.4. For the analyses with 10 and 20 meters overburden the upper and both these layers are suppressed/eliminated in the model respectively.

The lining is simulated as a shell in three dimensions by the definition of "skins" on solids, i.e. as the surfaces defining the excavation body.

As discussed above for the 2D models, framed areas in the focused region around the opening, (crown, invert and spring-lines) have been defined in the 3D model as well (see Figure 20 and Figure 22). Regarding this feature, the same descriptions provided for the 2D models are valid in the case of the 3D models too.

The model is divided to sets of geometrical definitions (Figure 25); these are 8 in total, being:

Soil material:

- Main soil bulk, extending from the bottom boundary to $10 \mathrm{~m}$ above the tunnel crown.

- Two additional soil layers each of $10 \mathrm{~m}$ thickness to simulate the different overburdens assumed in the investigations.

- The excavation of the tunnel.

Concrete / lining material:

- The lining.

- The breakout. 
- The above mentioned framed focus areas around the opening (one set for the frames at the crown and invert of the opening and one for the sides/springlines of the opening.

As mentioned above, these sets facilitate the handling of the model in the assignment of definitions and the model programming. As an example, the excavation and lining of the tunnel is programmed as a removal of the set of solid-elements comprising the soil excavation and placement of the set of shell-elements comprising the tunnel lining (including the opening elements). In turn the breakout is simulated as removal of the element set comprising the opening toward the child tunnel. The set definitions do also come handy in the post-processing, where areas of particular interest for the results (e.g. the defined spring-line frames) can be easily isolated and observed, or the results for these sets can be easily extracted in text form.

The materials used in these analyses are governed by simple isotropic linear elastic constitutive laws in line with the properties presented in 3.4.

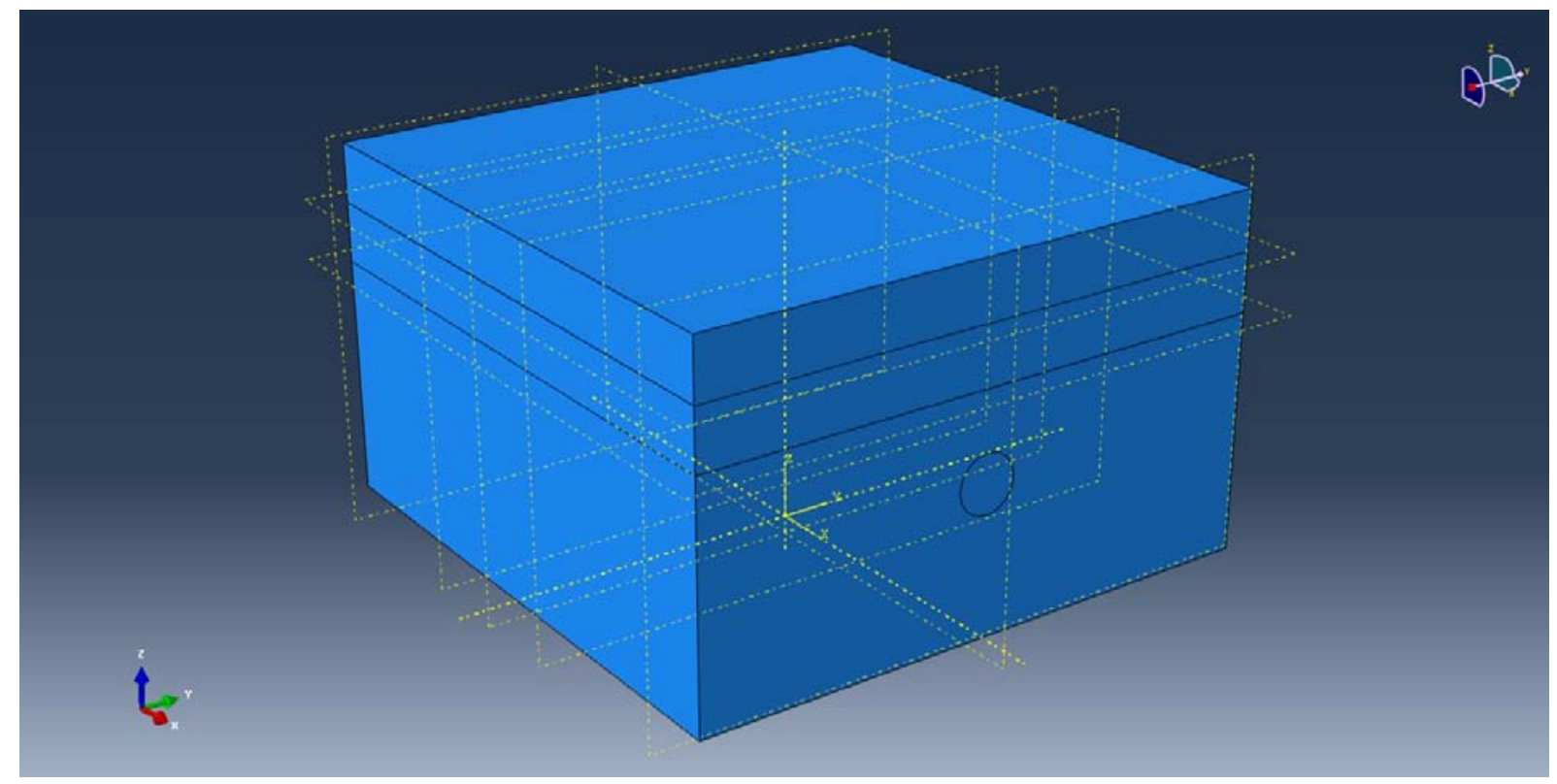

Figure 18: Overview of soil block for the 3D models 


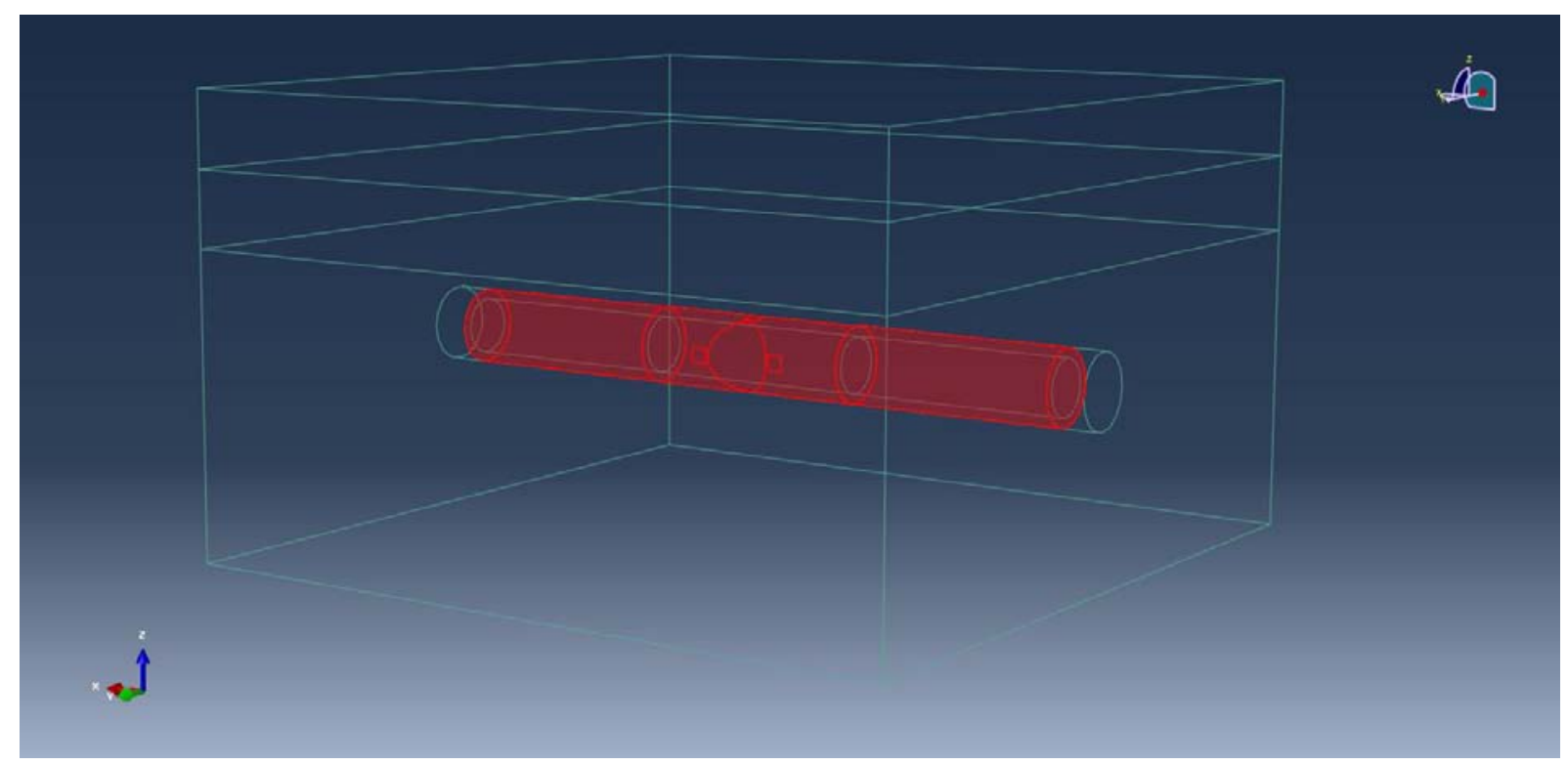

Figure 19: Overview of the 3D models including the tunnel structure, showing the lined tunnel in red

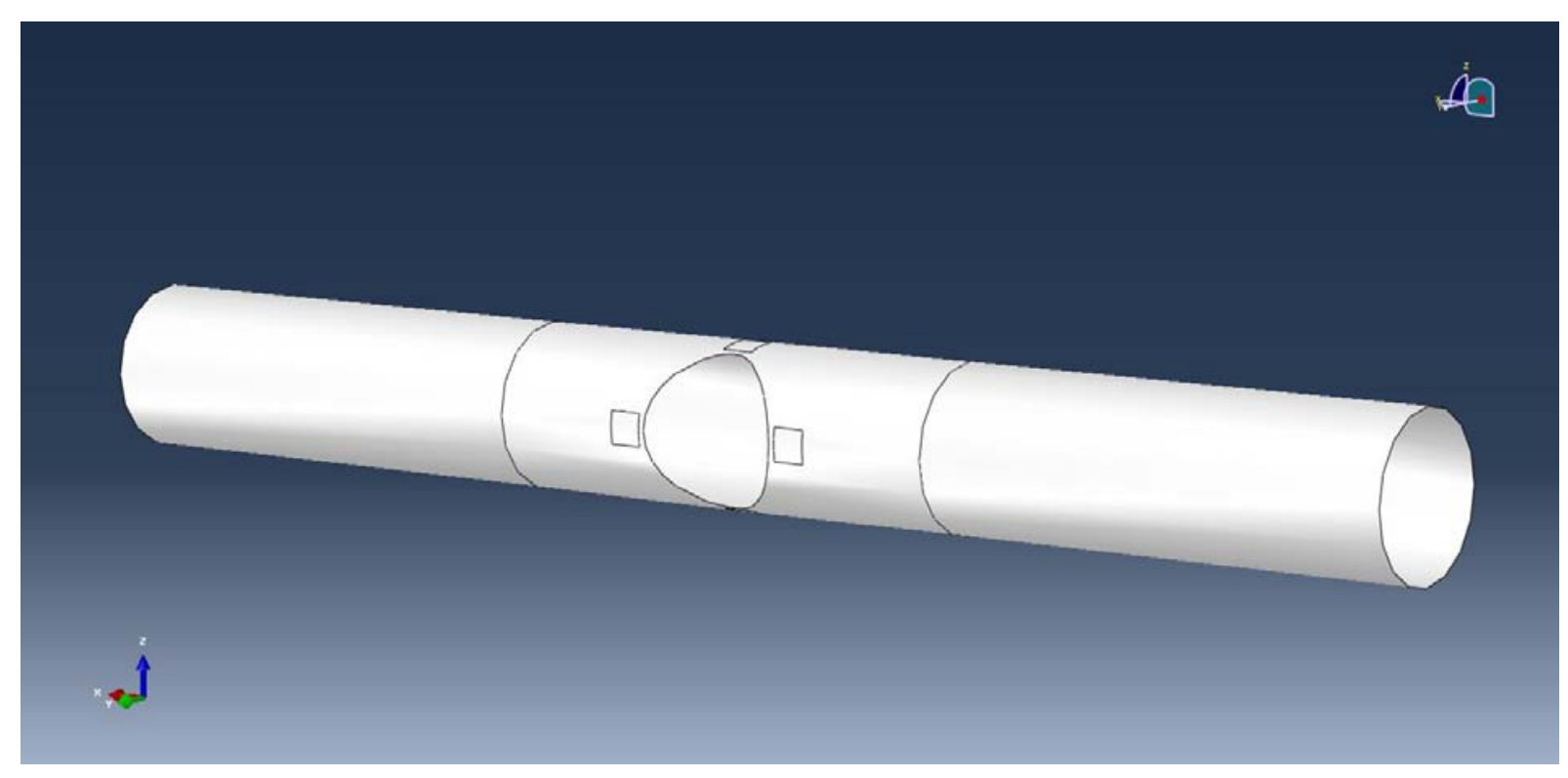

Figure 20: Detailed view of the modeled tunnel lining in the 3D model

For the meshing of the model a global element size of $8 \mathrm{~m}$ was applied for the soil, this being the element size towards the outer boundaries of the model typically seen in Figure 21. A generic size of $2 \mathrm{~m}$ was assigned to the lining shell elements (elements further from the opening region). The focus region around the openings used a smaller element size of approximately $0.35 \mathrm{~m}$ (equal to shell thickness). Within the excavation the mesh was optimized to be again coarser ( 2 to 3 meters) in order to decrease the number of elements 
and the computational effort (see Figure 22 and Figure 23). The elements used for the shells are as per the 2D models described in 3.5. The 3D soil elements were 4-noded tetrahedral linear solid elements specified as C3D4 in the Abaqus element library. For each 3D model the mesh comprised approximately 55000 solid (soil) elements and 6000 shell (lining) elements.

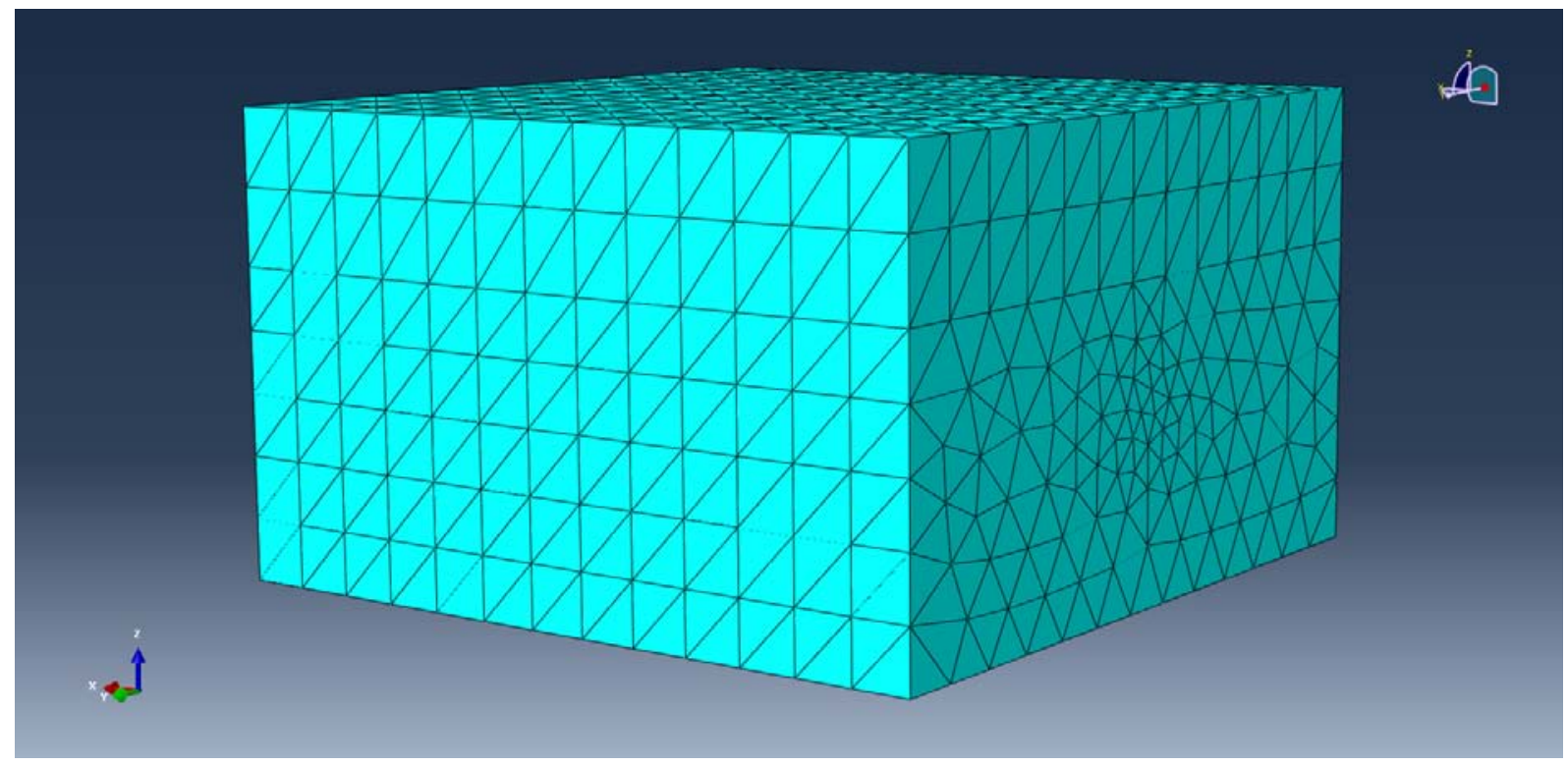

Figure 21: Overview of the mesh discretization of the soil block for the 3D models

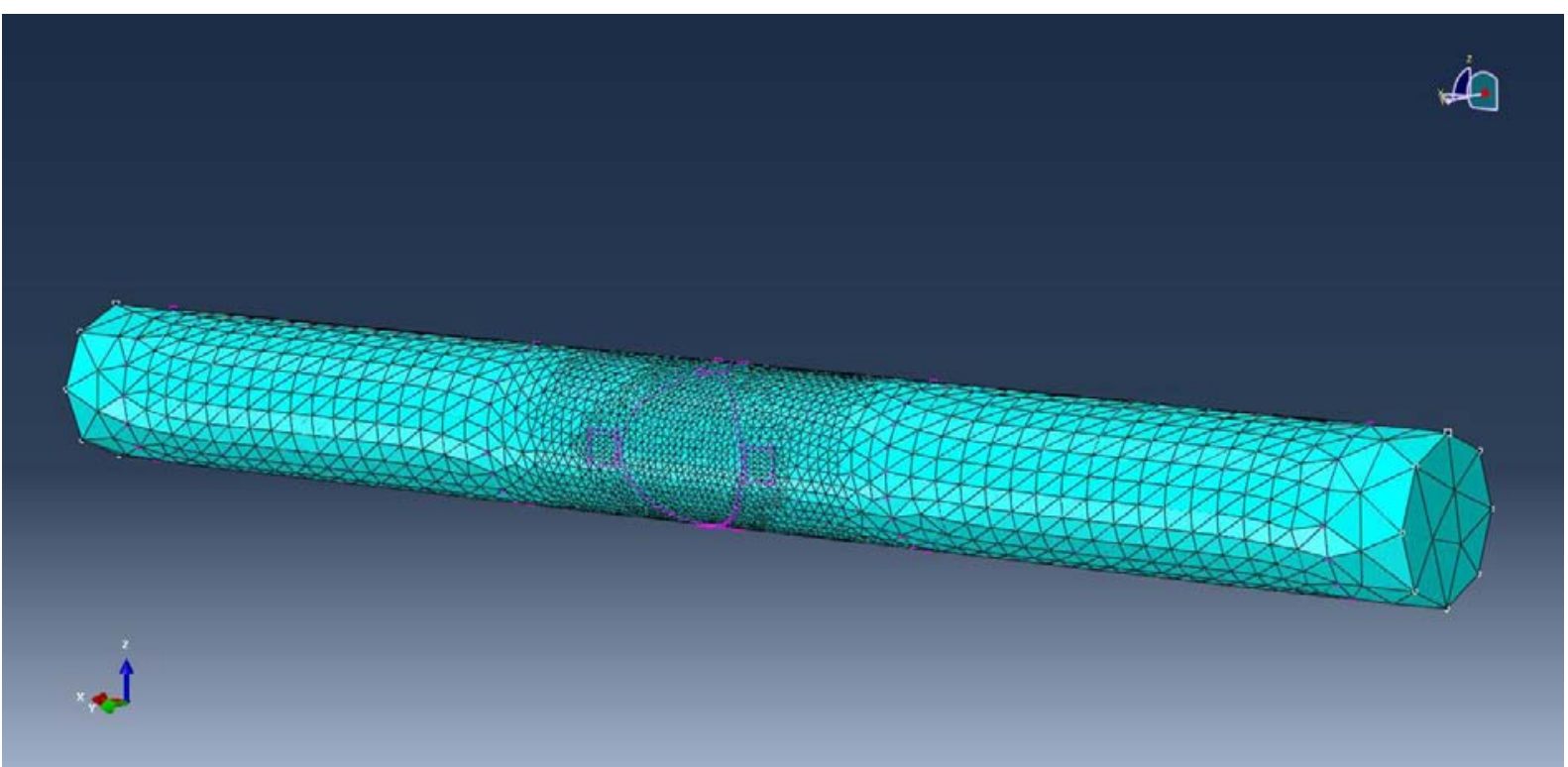

Figure 22: Overview of the mesh discretization of tunnel lining for the 3D models; purple indicates regions with local increased mesh density 


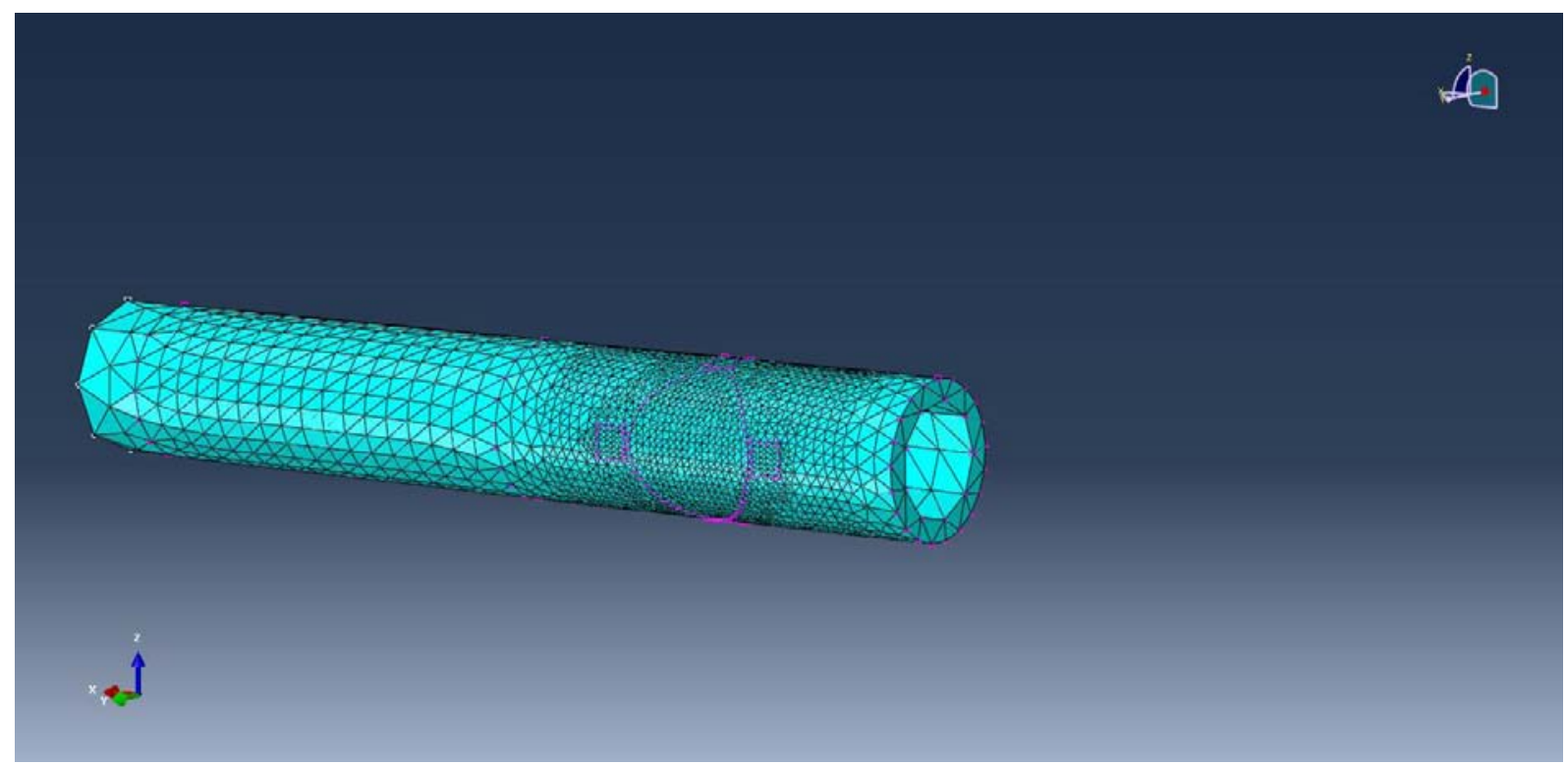

Figure 23: Detailed mesh plots of one of the 3D models with discretization refinement inside the tunnel lining

The stress in the model was defined as a stress field in the in-situ state of the soil in an individually programmed and calculated step (geostatic), since the program facilitates the input of a geostatic stress field as an initial stress state. The earth pressure coefficient value was assumed $\mathrm{K}_{0}=1$. The magnitude of the stresses in the soil was calculated automatically based on the input density of the soil and the magnitude and direction of gravity in the model (assumed $10 \mathrm{~m} / \mathrm{s}^{2}$ ). The boundary conditions assigned were displacement restraints against the $x, y, z$ directions in the respective boundary sides of the model (Figure 24).

Three steps in total were programmed in the model, these being:

1. The in-situ geostatic stress and equilibrium calculation

2. The excavation of the tunnel and the introduction of the lining (wished-in-place without precedent softening), and

3. The removal of the breakout which generated the stress redistribution around the opening.

These functions were manually defined by editing the input text file of each analyzed model. 
The output of the model was calibrated to produce only purpose-relevant values, these being mainly deformations and elastic strains, stresses, and section forces (axial, moment, shear) in the shell. The analysis procedure retained the default Abaqus solver calibration as per the 2D plate models described in 3.5 .

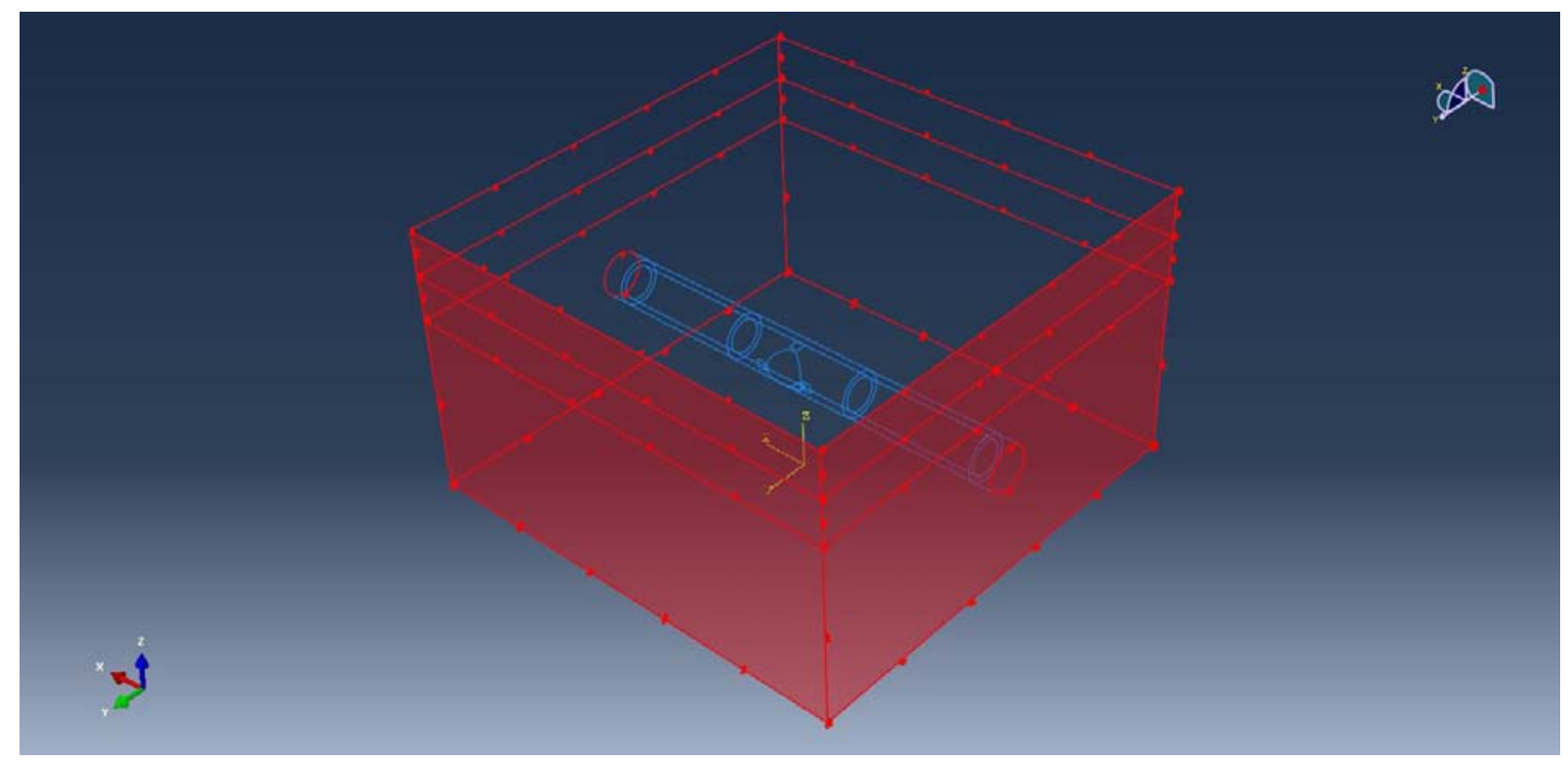

Figure 24: Overview of boundary conditions for one of the 3D models

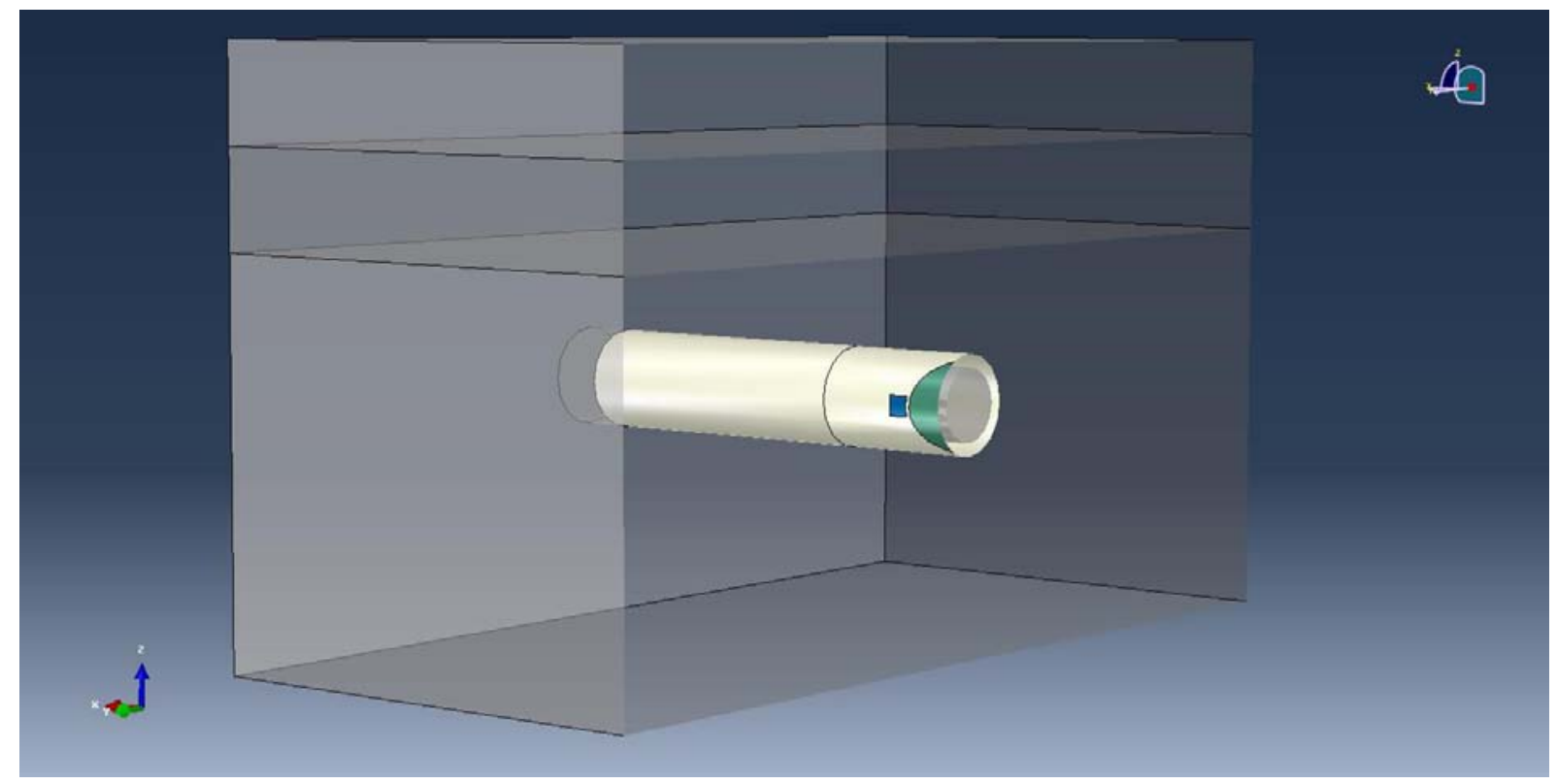

Figure 25: Overview of sets for one of the 3D models 


\section{$4 \quad$ Results}

\subsection{Output parameters}

The parameters requested as output to be processed in the presented investigations are

A. For the state of completed excavation and complete primary lining installation the Average Hoop and Longitudinal Forces, at the mid-span of the modeled tunnel

B. For the state when the opening is created, the design-governing maxima/minima of the Hoop Forces and Moments, and the Longitudinal Forces and Moments at four locations around the opening as discussed above: Crown, Invert, Left and Right Springline.

Due to the symmetrical effects and for ease of processing, the results are grouped for the locations of (i) the crown and the invert (approximately equal and averaged from the two locations) and (ii) the springlines (equal due to symmetry).

Furthermore, the results of state (B) are normalized by dividing them with the average hoop force of state (A), the latter one assumed to be the main agent of stress within the lining, which is to be redistributed after the breakout. This is decided based on the anticipated proportionality between the section forces and moments around the opening with the hoop forces in the parent tunnel prior to breakout as discussed in 2.2.1.

\subsection{Typical results and interpretation}

The entire register of results may be found in Table 5 and Table 6. Selected result plots (i.e. illustrated distributions of the section forces/moments around the openings) are given in this section for the models listed below. 


\begin{tabular}{|c|c|c|c|c|c|c|c|c|c|c|}
\hline Sr.Nr & $\begin{array}{c}\text { Avg. Hoop Force } \\
\text { at Ring Closure } \\
{[\mathrm{kN}]}\end{array}$ & $\begin{array}{l}\text { Avg. Longitudinal } \\
\text { Force at Ring } \\
\text { Closure [kN] }\end{array}$ & $\begin{array}{l}\text { Max. Hoop } \\
\text { Force } \\
\text { Crown/invert } \\
{[\mathrm{kN}]}\end{array}$ & $\begin{array}{c}\text { Min Hoop } \\
\text { Moment } \\
\text { Crown/invert } \\
{[\mathrm{kNm}] \text { (blue) }}\end{array}$ & $\begin{array}{c}\text { Max } \\
\text { Longitudinal } \\
\text { Force } \\
\text { Crown/invert } \\
{[\mathrm{kN}]}\end{array}$ & $\begin{array}{l}\text { Max Longitudinal } \\
\text { Moment } \\
\text { Crown/invert } \\
{[\mathrm{kNm}]}\end{array}$ & $\begin{array}{l}\text { Min Hoop } \\
\text { Force } \\
\text { Springlines } \\
{[\mathrm{kN}]}\end{array}$ & $\begin{array}{l}\text { Min. Hoop } \\
\text { Moment } \\
\text { Springlines } \\
{[\mathrm{kNm}]}\end{array}$ & $\begin{array}{c}\text { Max } \\
\text {.Longitudinal } \\
\text { Force Springlines } \\
{[\mathrm{kN}]}\end{array}$ & $\begin{array}{l}\text { Min. } \\
\text { Longitudinal } \\
\text { Moment } \\
\text { Springlines } \\
\text { [kNm] (blue) }\end{array}$ \\
\hline & & & $\mathrm{SF} 2-\mathrm{Cl}$ & SM2-Cl & SF1-Cl & SM1-Cl & SF2-SP & SM2-SP & SF1-SP & SM1-SP \\
\hline 1 & -3404 & -719 & -856 & -111 & -483 & 306 & -10180 & -188 & -1254 & -183 \\
\hline 2 & -3340 & -706 & -817 & -75 & -560 & 205 & -9506 & -105 & -1096 & -153 \\
\hline 3 & -3212 & -679 & -772 & -41 & -469 & 121 & -8405 & -56 & -911 & -116 \\
\hline 4 & -2433 & -466 & -500 & -83 & 481 & 230 & -7253 & -127 & -879 & -130 \\
\hline 5 & -2387 & -340 & -504 & -55 & 132 & 153 & -6766 & -71 & -765 & -109 \\
\hline 6 & -2383 & -381 & -508 & -20 & -59 & 97 & -6638 & -57 & -707 & -92 \\
\hline 7 & -1444 & -175 & -275 & -60 & 414 & 139 & -4174 & -58 & -493 & -74 \\
\hline 8 & -1414 & -192 & -275 & -39 & 141 & 93 & -3896 & -33 & -431 & -62 \\
\hline 9 & -1364 & -214 & -272 & -21 & -5 & 55 & -3445 & -19 & -358 & -47 \\
\hline 10 & & $\neq 0$ & & & & & & & & \\
\hline 11 & -1000 & 0 & 87 & N.A. & 882 & N.A. & -2870 & N.A. & -163 & N.A. \\
\hline 12 & -1000 & 0 & 41 & N.A. & 682 & N.A. & -2543 & N.A. & -180 & N.A. \\
\hline 13 & -3410 & -471 & -736 & -98 & 1163 & 360 & -10330 & -246 & -1414 & -178 \\
\hline 14 & -3350 & -526 & -756 & -70 & 667 & 241 & -9568 & -139 & -1236 & -143 \\
\hline 15 & -3222 & -555 & -750 & -41 & 319 & 141 & -8453 & -69 & -1018 & -106 \\
\hline 16 & -2390 & -383 & -519 & -77 & 836 & 256 & -7266 & -162 & -995 & -124 \\
\hline 17 & -2430 & -342 & -519 & -53 & 480 & 172 & -6731 & -93 & -867 & -100 \\
\hline 18 & -2300 & -406 & -515 & -31 & 230 & 101 & -5950 & -46 & -713 & -75 \\
\hline 19 & -1435 & -192 & -276 & -59 & 530 & 149 & -4136 & -74 & -564 & -69 \\
\hline 20 & -1408 & -214 & -277 & -39 & 307 & 101 & -3833 & -44 & -488 & -56 \\
\hline 21 & -1360 & -231 & -276 & -22 & 151 & 60 & -3393 & -22 & -402 & -42 \\
\hline 22 & & $\neq 0$ & & & & & & & & \\
\hline 23 & -1000 & 0 & 57 & N.A. & 734 & N.A. & -2680 & N.A. & -183 & N.A. \\
\hline 24 & -1000 & 0 & 41 & N.A. & 632 & N.A. & -2468 & N.A. & -205 & N.A. \\
\hline 25 & -3375 & -378 & -773 & -60 & 1424 & 383 & -10340 & -280 & -1595 & -187 \\
\hline 26 & -3354 & -526 & -741 & -48 & 852 & 265 & -9490 & -169 & -1387 & -146 \\
\hline 27 & -3212 & -536 & -708 & -31 & 420 & 159 & -8338 & -88 & -1145 & -105 \\
\hline 28 & -2385 & -291 & -532 & -48 & 1028 & 272 & -7293 & -188 & -1119 & -132 \\
\hline 29 & -2639 & -317 & -508 & -37 & 612 & 189 & -6694 & -115 & -974 & -103 \\
\hline 30 & -2288 & -378 & -486 & -23 & 301 & 113 & -5883 & -60 & -804 & -74 \\
\hline 31 & -1420 & -162 & -287 & -36 & 650 & 159 & -4191 & -92 & -634 & -75 \\
\hline 32 & -1393 & -201 & -272 & -26 & 382 & 111 & -3847 & -58 & -553 & -59 \\
\hline 33 & -1342 & -218 & -260 & -16 & 188 & 67 & -3386 & -31 & -458 & -42 \\
\hline 34 & & $\neq 0$ & & & & & & & & \\
\hline 35 & -1000 & 0 & 36 & N.A. & 581 & N.A. & -2490 & N.A. & -218 & N.A. \\
\hline 36 & -1000 & 0 & 40 & N.A. & 564 & N.A. & -2365 & N.A. & -238 & N.A. \\
\hline 37 & -3334 & -529 & -550 & 22 & -413 & 134 & -10450 & -73 & -781 & -365 \\
\hline 38 & -3266 & -492 & -480 & 40 & -484 & 144 & -8816 & -50 & -7962 & -305 \\
\hline
\end{tabular}

Table 5: Summary of results from the various analysis (also refer to Table 4 and Figure 26)

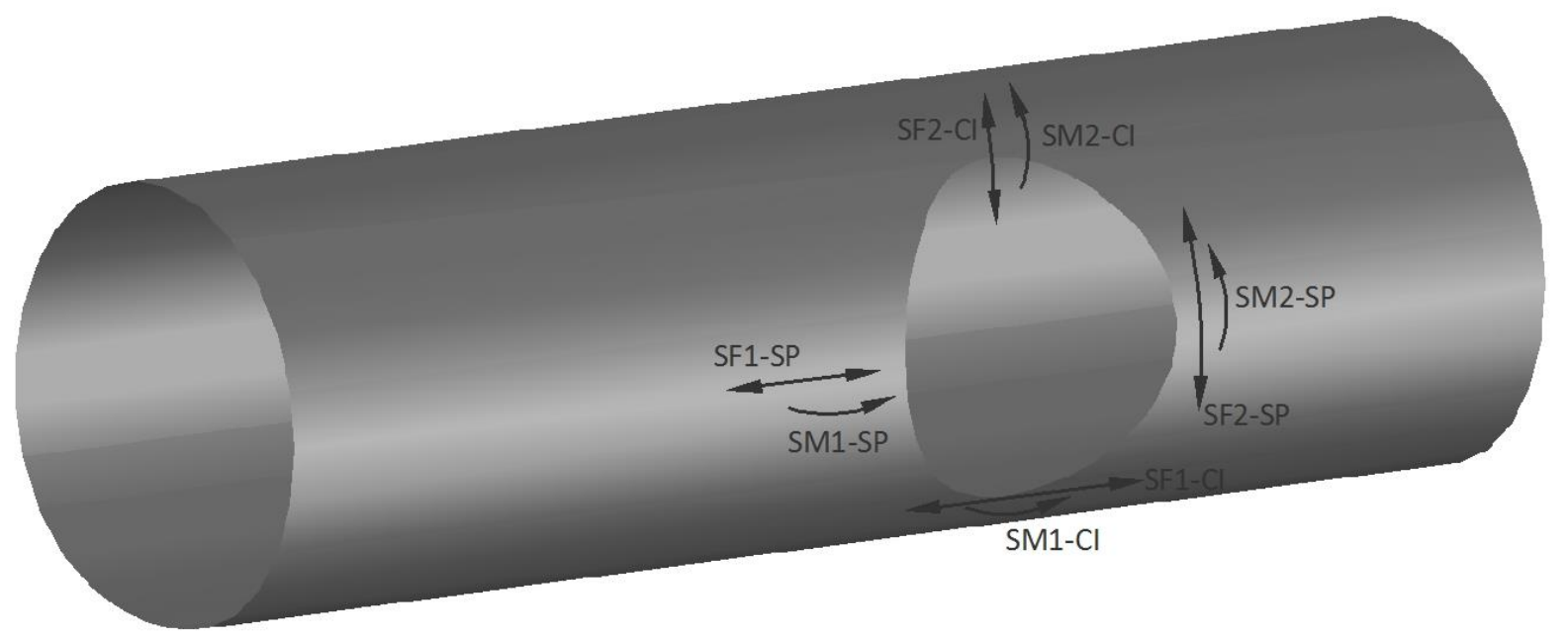

Figure 26: Notation of monitored section forces around the openings 


\begin{tabular}{|c|c|c|c|c|c|c|c|c|}
\hline Sr.Nr & $\begin{array}{l}\text { Ratio of Hoop Force } \\
\text { at ring closure to } \\
\text { Hoop Force at } \\
\text { Crown/invert after } \\
\text { breakout }\end{array}$ & $\begin{array}{c}\text { Ratio of Hoop Force } \\
\text { at ring closure to } \\
\text { Hoop Moment at } \\
\text { Crown/invert after } \\
\text { breakout }\end{array}$ & $\begin{array}{c}\text { Ratio of Hoop Force } \\
\text { at ring closure to } \\
\text { Longitudinal Force } \\
\text { at Crown/invert after } \\
\text { breakout }\end{array}$ & $\begin{array}{c}\text { Ratio of Hoop Force at } \\
\text { ring closure to } \\
\text { Longitudinal Moment } \\
\text { at Crown/invert after } \\
\text { breakout }\end{array}$ & $\begin{array}{l}\text { Ratio of Hoop Force } \\
\text { at ring closure to } \\
\text { Hoop Force at } \\
\text { Springlines after } \\
\text { breakout }\end{array}$ & $\begin{array}{l}\text { Ratio of Hoop Force } \\
\text { at ring closure to } \\
\text { Hoop Moment at } \\
\text { Springlines after } \\
\text { breakout }\end{array}$ & $\begin{array}{c}\text { Ratio of Hoop Force } \\
\text { at ring closure to } \\
\text { Longitudinal Force } \\
\text { at Springlines after } \\
\text { breakout }\end{array}$ & $\begin{array}{c}\text { Ratio of Hoop Force at } \\
\text { ring closure to } \\
\text { Longitudinal Moment } \\
\text { at Springlines after } \\
\text { breakout }\end{array}$ \\
\hline & sf2-Cl-norm & sm2-Cl-norm & sf1-Cl-norm & sm1-Cl-norm & sf2-SP-norm & sm2-SP-norm & sf1-SP-norm & sm1-SP-norm \\
\hline 1 & 0.251 & 0.033 & 0.142 & -0.090 & 2.991 & 0.055 & 0.368 & 0.054 \\
\hline 2 & 0.245 & 0.022 & 0.168 & -0.061 & 2.846 & 0.031 & 0.328 & 0.046 \\
\hline 3 & 0.240 & 0.013 & 0.146 & -0.038 & 2.617 & 0.017 & 0.284 & 0.036 \\
\hline 4 & 0.206 & 0.034 & -0.198 & -0.095 & 2.981 & 0.052 & 0.361 & 0.053 \\
\hline 5 & 0.211 & 0.023 & -0.055 & -0.064 & 2.835 & 0.030 & 0.320 & 0.046 \\
\hline 6 & 0.213 & 0.008 & 0.025 & -0.041 & 2.786 & 0.024 & 0.297 & 0.039 \\
\hline 7 & 0.190 & 0.042 & -0.287 & -0.096 & 2.891 & 0.040 & 0.341 & 0.051 \\
\hline 8 & 0.194 & 0.028 & -0.100 & -0.066 & 2.755 & 0.023 & 0.305 & 0.044 \\
\hline 9 & 0.199 & 0.015 & 0.004 & -0.040 & 2.526 & 0.014 & 0.262 & 0.034 \\
\hline 10 & 0.217 & 0.024 & -0.017 & -0.066 & 2.803 & 0.032 & 0.319 & 0.045 \\
\hline 11 & -0.087 & N.A. & -0.882 & N.A. & 2.870 & N.A. & 0.163 & N.A. \\
\hline 12 & -0.041 & N.A. & -0.682 & N.A. & 2.543 & N.A. & 0.180 & N.A. \\
\hline 13 & 0.216 & 0.029 & -0.341 & -0.106 & 3.029 & 0.072 & 0.415 & 0.052 \\
\hline 14 & 0.226 & 0.021 & -0.199 & -0.072 & 2.856 & 0.041 & 0.369 & 0.043 \\
\hline 15 & 0.233 & 0.013 & -0.099 & -0.044 & 2.624 & 0.021 & 0.316 & 0.033 \\
\hline 16 & 0.217 & 0.032 & -0.350 & -0.107 & 3.040 & 0.068 & 0.416 & 0.052 \\
\hline 17 & 0.214 & 0.022 & -0.198 & -0.071 & 2.770 & 0.038 & 0.357 & 0.041 \\
\hline 18 & 0.224 & 0.013 & -0.100 & -0.044 & 2.587 & 0.020 & 0.310 & 0.033 \\
\hline 19 & 0.192 & 0.041 & -0.369 & -0.104 & 2.882 & 0.052 & 0.393 & 0.048 \\
\hline 20 & 0.197 & 0.028 & -0.218 & -0.072 & 2.722 & 0.031 & 0.347 & 0.040 \\
\hline 21 & 0.203 & 0.016 & -0.111 & -0.044 & 2.495 & 0.016 & 0.296 & 0.031 \\
\hline 22 & 0.213 & 0.024 & -0.221 & -0.074 & 2.778 & 0.040 & 0.358 & 0.041 \\
\hline 23 & -0.057 & N.A. & -0.734 & N.A. & 2.680 & N.A. & 0.183 & N.A. \\
\hline 24 & -0.041 & N.A. & -0.632 & N.A. & 2.468 & N.A. & 0.205 & N.A. \\
\hline 25 & 0.229 & 0.018 & -0.422 & -0.113 & 3.064 & 0.083 & 0.473 & 0.055 \\
\hline 26 & 0.221 & 0.014 & -0.254 & -0.079 & 2.829 & 0.050 & 0.414 & 0.044 \\
\hline 27 & 0.220 & 0.010 & -0.131 & -0.050 & 2.596 & 0.027 & 0.356 & 0.033 \\
\hline 28 & 0.223 & 0.020 & -0.431 & -0.114 & 3.058 & 0.079 & 0.469 & 0.055 \\
\hline 29 & 0.192 & 0.014 & -0.232 & -0.072 & 2.537 & 0.044 & 0.369 & 0.039 \\
\hline 30 & 0.212 & 0.010 & -0.132 & -0.049 & 2.571 & 0.026 & 0.351 & 0.032 \\
\hline 31 & 0.202 & 0.025 & -0.458 & -0.112 & 2.951 & 0.065 & 0.446 & 0.053 \\
\hline 32 & 0.195 & 0.019 & -0.274 & -0.080 & 2.762 & 0.042 & 0.397 & 0.042 \\
\hline 33 & 0.194 & 0.012 & -0.140 & -0.050 & 2.523 & 0.023 & 0.341 & 0.031 \\
\hline 34 & 0.210 & 0.016 & -0.275 & -0.080 & 2.766 & 0.049 & 0.402 & 0.043 \\
\hline 35 & -0.036 & N.A. & -0.581 & N.A. & 2.490 & N.A. & 0.218 & N.A. \\
\hline 36 & -0.040 & N.A. & -0.564 & N.A. & 2.365 & N.A. & 0.238 & N.A. \\
\hline 37 & 0.165 & -0.007 & 0.124 & -0.040 & 3.134 & 0.022 & 0.234 & 0.109 \\
\hline 38 & 0.147 & -0.012 & 0.148 & -0.044 & 2.699 & 0.015 & 2.438 & 0.093 \\
\hline
\end{tabular}

Table 6: Summary of normalized results (divided with average hoop force at ring closure) from the various analysis (also refer to Table 4 and Table 5)

List of models, of which results screenshots are presented in Section 4.2:

Child-Parent ratio $\mathrm{d} / \mathrm{D}=0.90$ :

Model Nr.1: 3D-25-35-090

Model Nr.10: 2D-35-090

Child-Parent ratio $d / D=0.75$ :

Model Nr.20: 3D-50-25-075

Model Nr.26: 2D-25-075

Child-Parent ratio $d / D=0.60$ :

Model Nr.39: 3D-100-15-060

Model Nr.42: 2D-15-060 


\subsubsection{Interpretation and behavior in 2D}

As seen in the figures below, the 2D results for circular openings do not pose a definite agreement with the analytical solutions of Kirsch. These analytical solutions seem to capture only the trend of the stress distribution around the opening. It is evident that for the investigated cases the analytical solutions can be somewhat more conservative (show higher stresses) but not when it comes to the stress concentration at the opening springlines. This may partially be attributed to numerical issues, as already discussed in (Jones 2013), but it is mostly the shape of the opening that dictates the stress flow around it. Consequently also the size of the child tunnel, i.e. the developed (unwrapped) geometry, comes to play a role on the consistency of the plane stress results with the Kirsch solutions.

In particular, it is being evident that the hoop forces at the crown and invert are overestimated by up to $50 \%$ by the analytical approach (Figure 33). However these remain at a relatively quite low stress level, perhaps irrelevant to design or feasibility aspects. The Kirsch solution for round openings also overestimates the longitudinal forces at the springlines by some $10 \%$ (Figure 36 ), and moreover both the 2D numerical and the analytical methods yield a compressive stress state at these locations. When it comes to values that are more relevant for the design the Kirsch solution underestimates the compressive forces to be expected at the springlines (Figure 35); in this case additional thickening or a similar design provision should theoretically be safeguarded to bear and redistribute these stress concentrations. On the other side, the analytical solutions seem to overestimate the longitudinal forces by up to $20 \%$ at crown and invert (Figure 34 ), which are typically a main element design. Consequently, a "Kirsch-based" design would lead to superfluous reinforcement, with certain disadvantages for the budget and moreover for the constructability and construction safety. In any case, it needs to be highlighted that these numbers, i.e. deviations between the 2D plane stress numerical solution and the simplified circular-hole analytical solution may substantially vary, depending on the opening geometries as seen in the graphs below and the results summary in Table 6 . 


\section{Results of Model Nr.10: 2D-090}

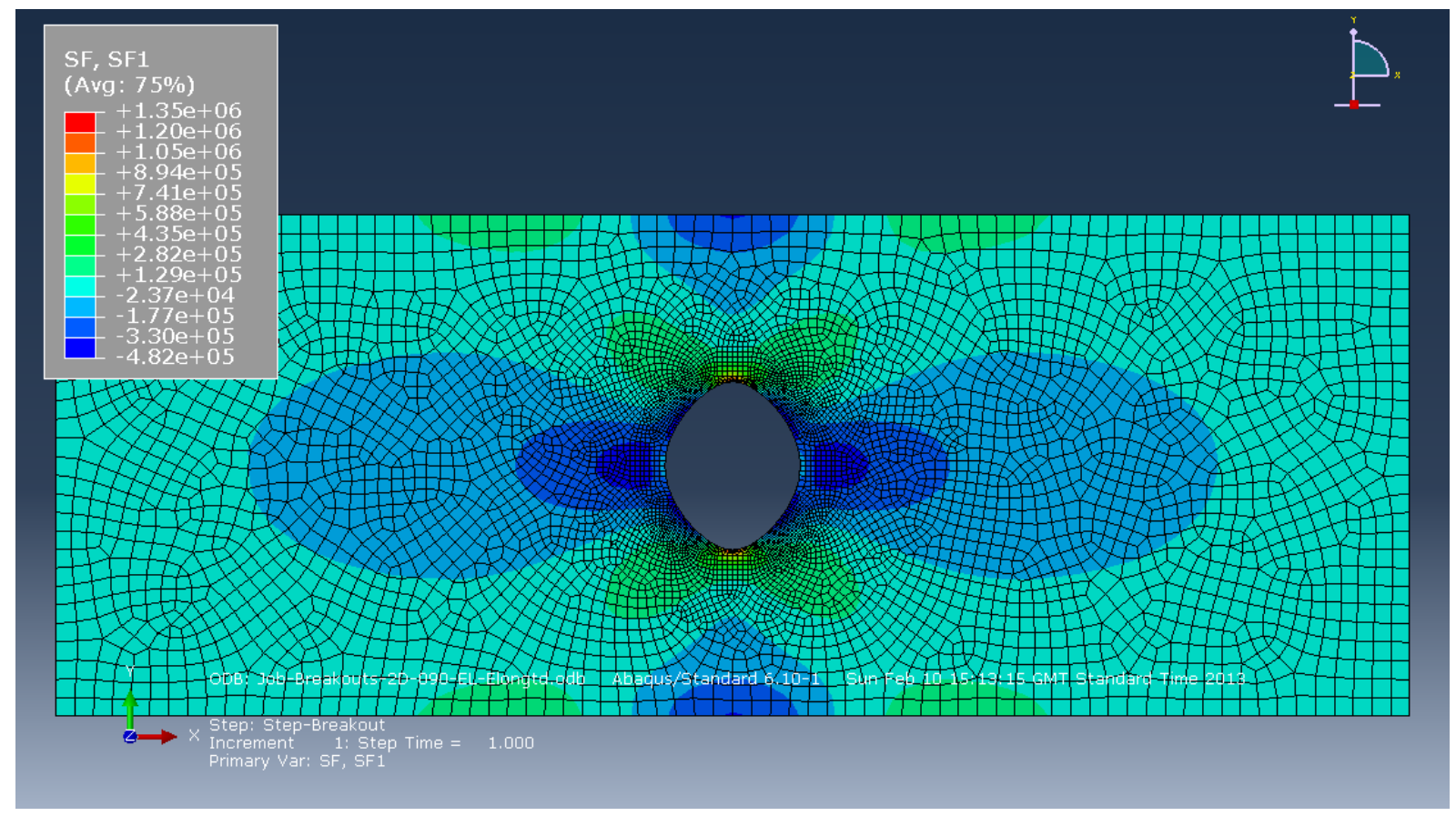

Figure 27: Model Nr.10: 2D-090; SF1: Longitudinal forces

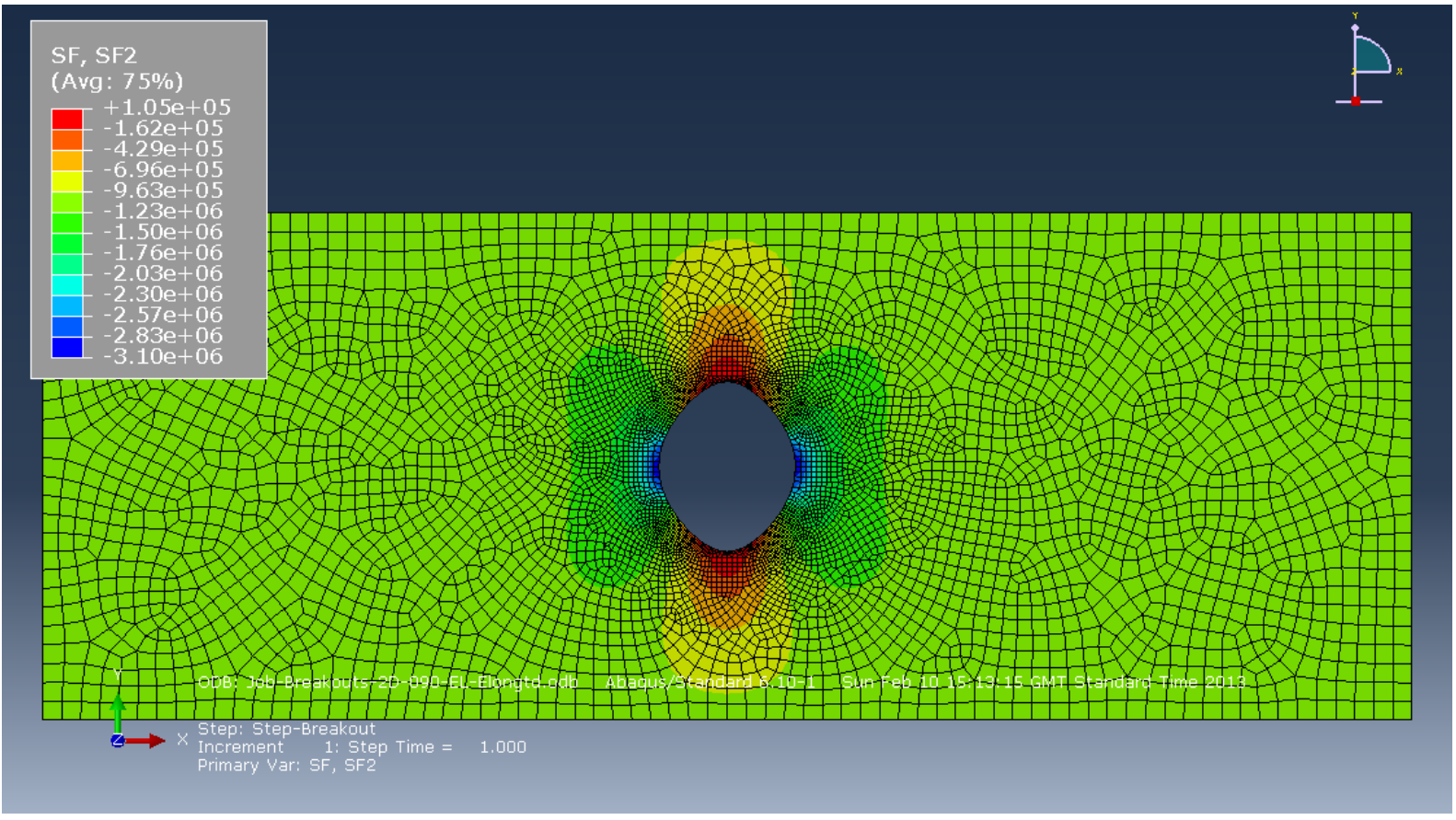

Figure 28: Model Nr.10: 2D-090; SF2: Hoop forces 
Results of Model Nr.26: 2D-075

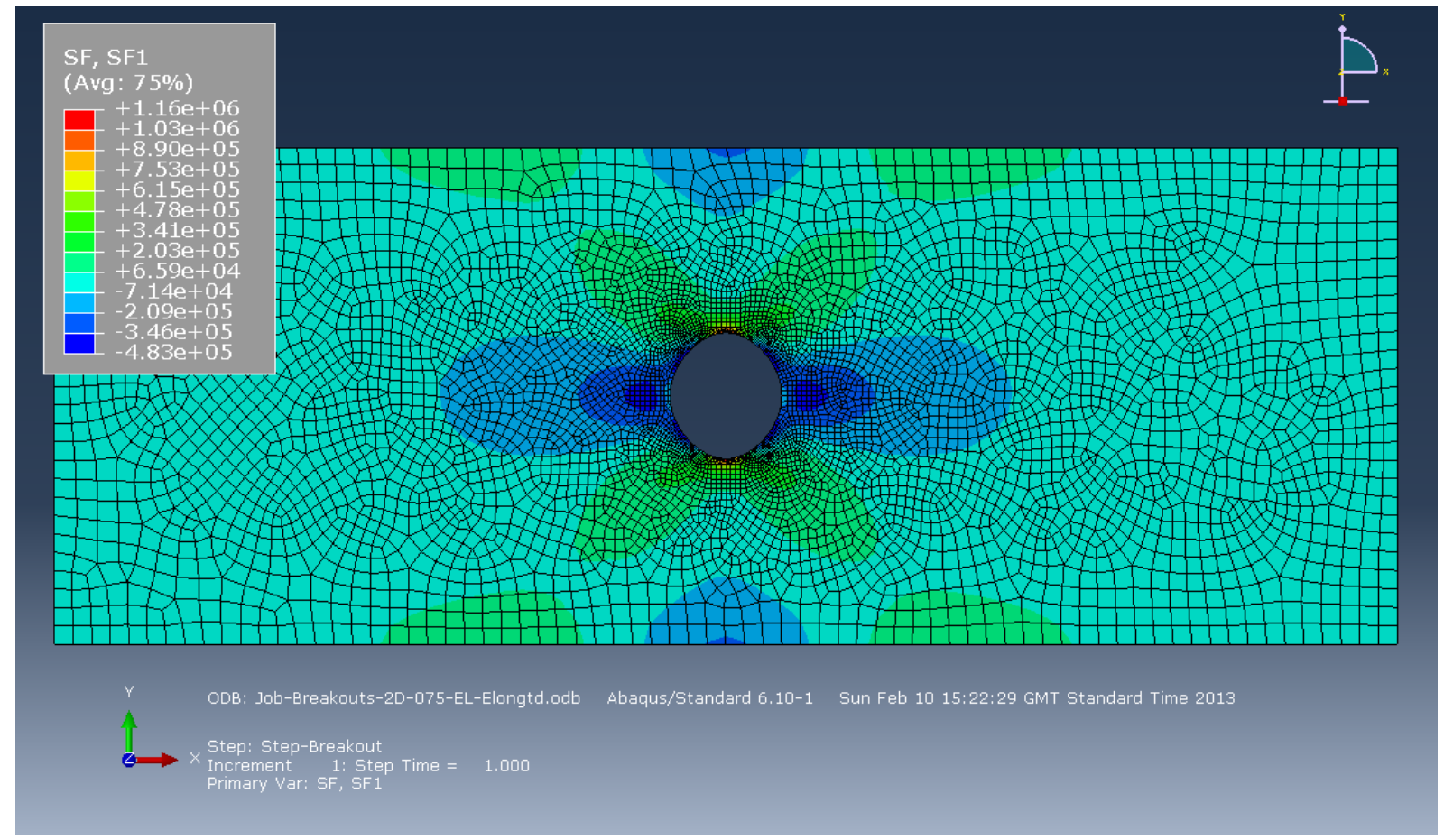

Figure 29: Model Nr.26: 2D-075; SF1: Longitudinal forces

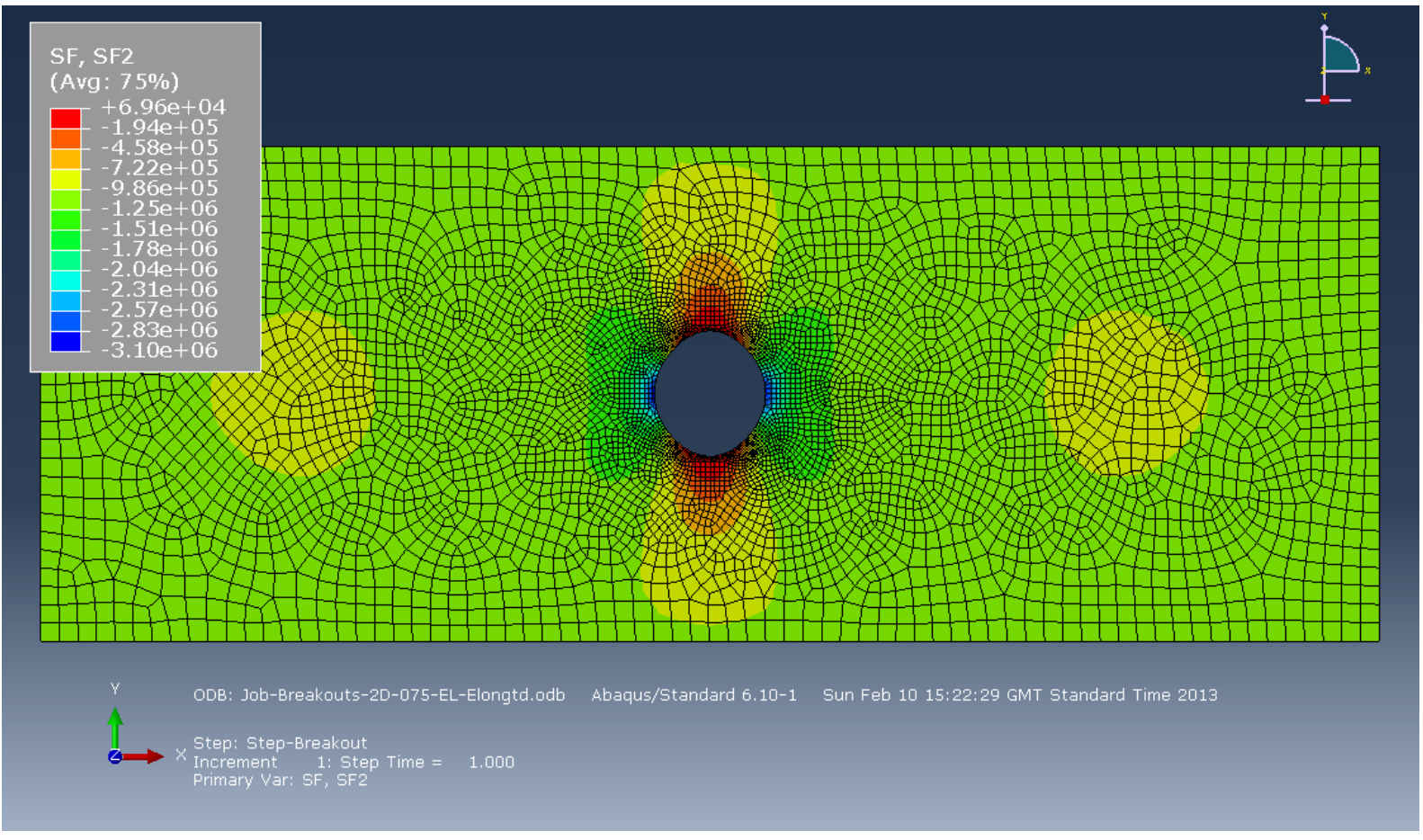

Figure 30: Model Nr.26: 2D-075; SF2: Hoop forces 
Results of Model Nr.42: 2D-060

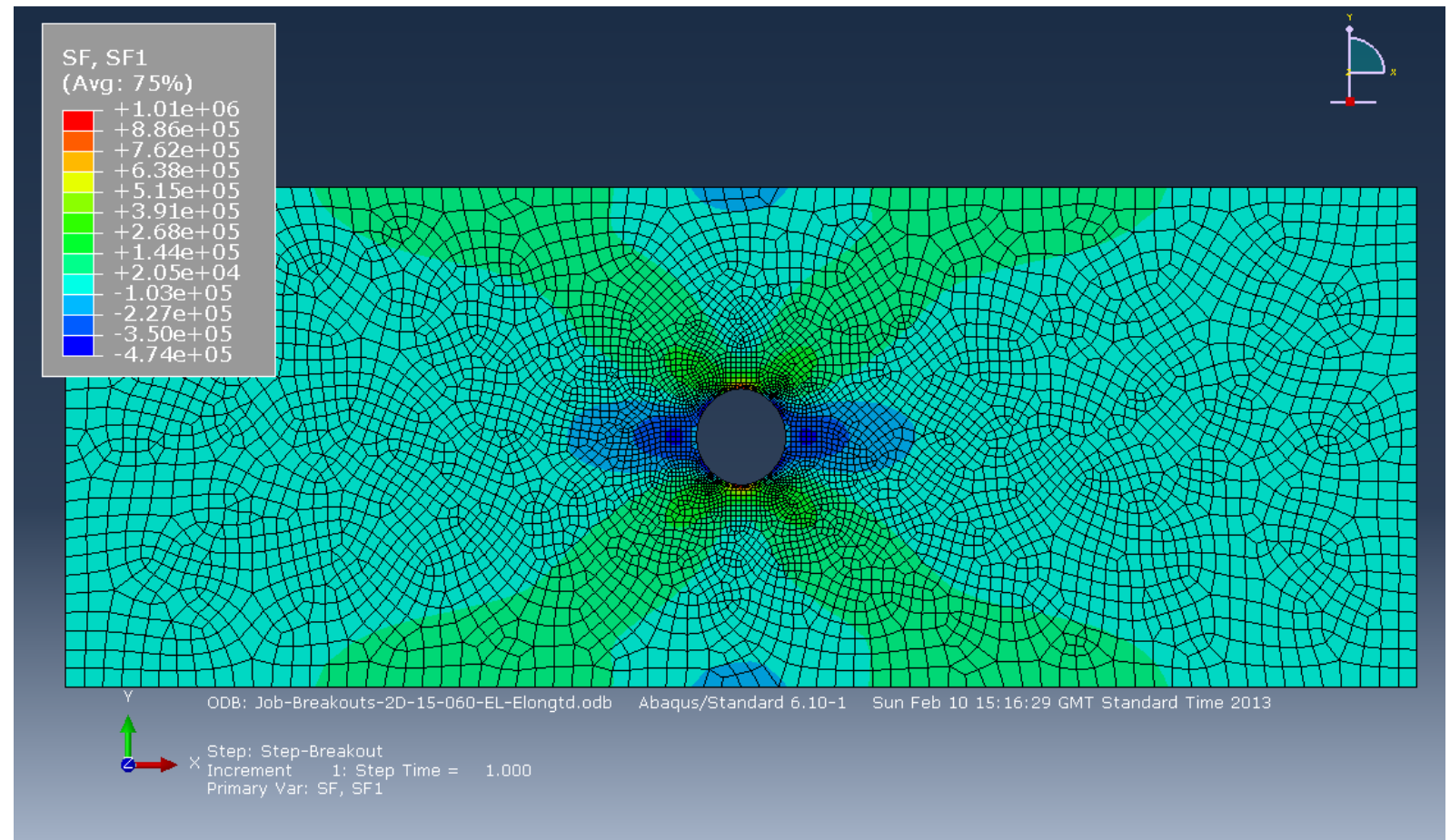

Figure 31: Model Nr.42: 2D-060; SF1: Longitudinal forces

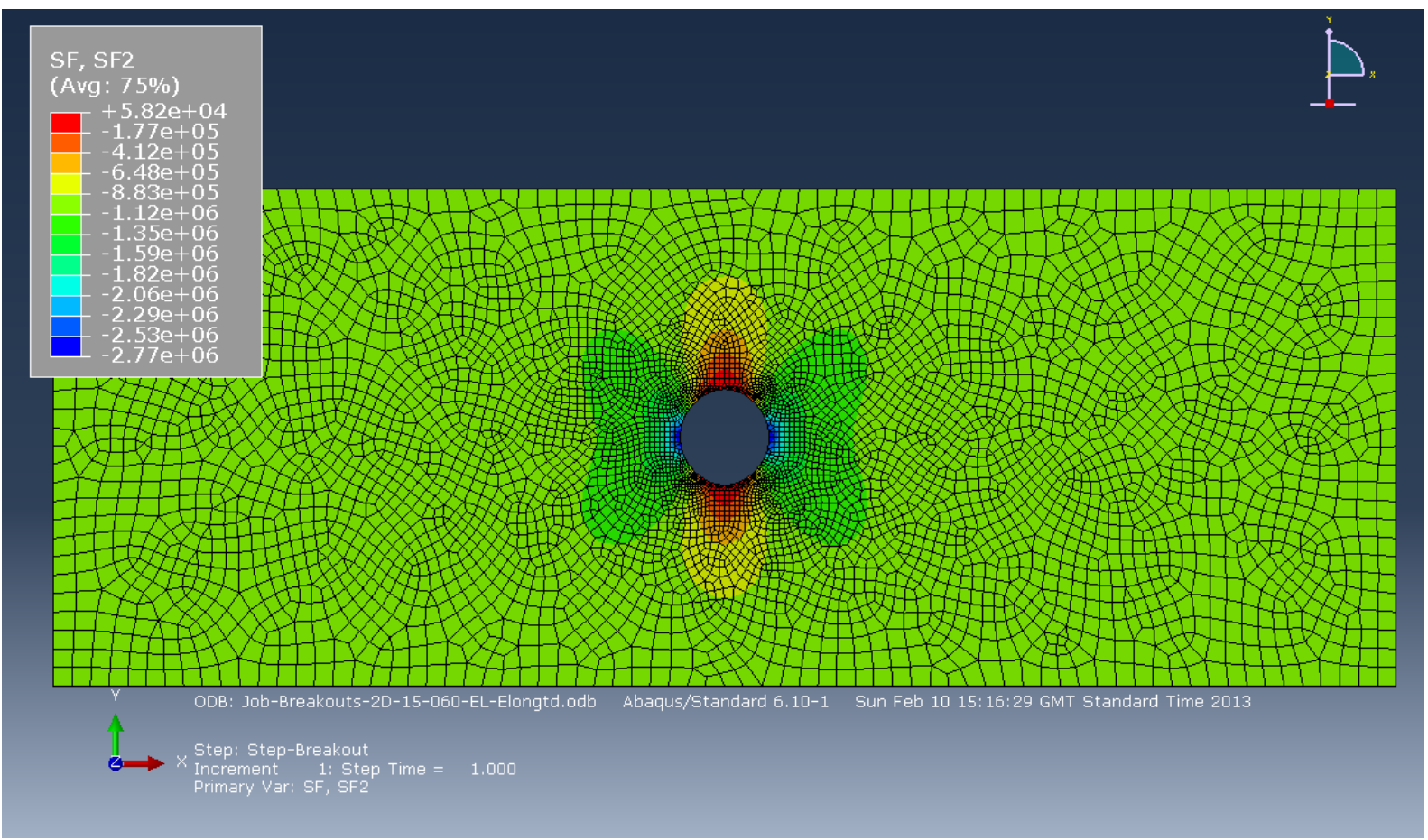

Figure 32: Model Nr.42: 2D-060; SF2: Hoop forces 


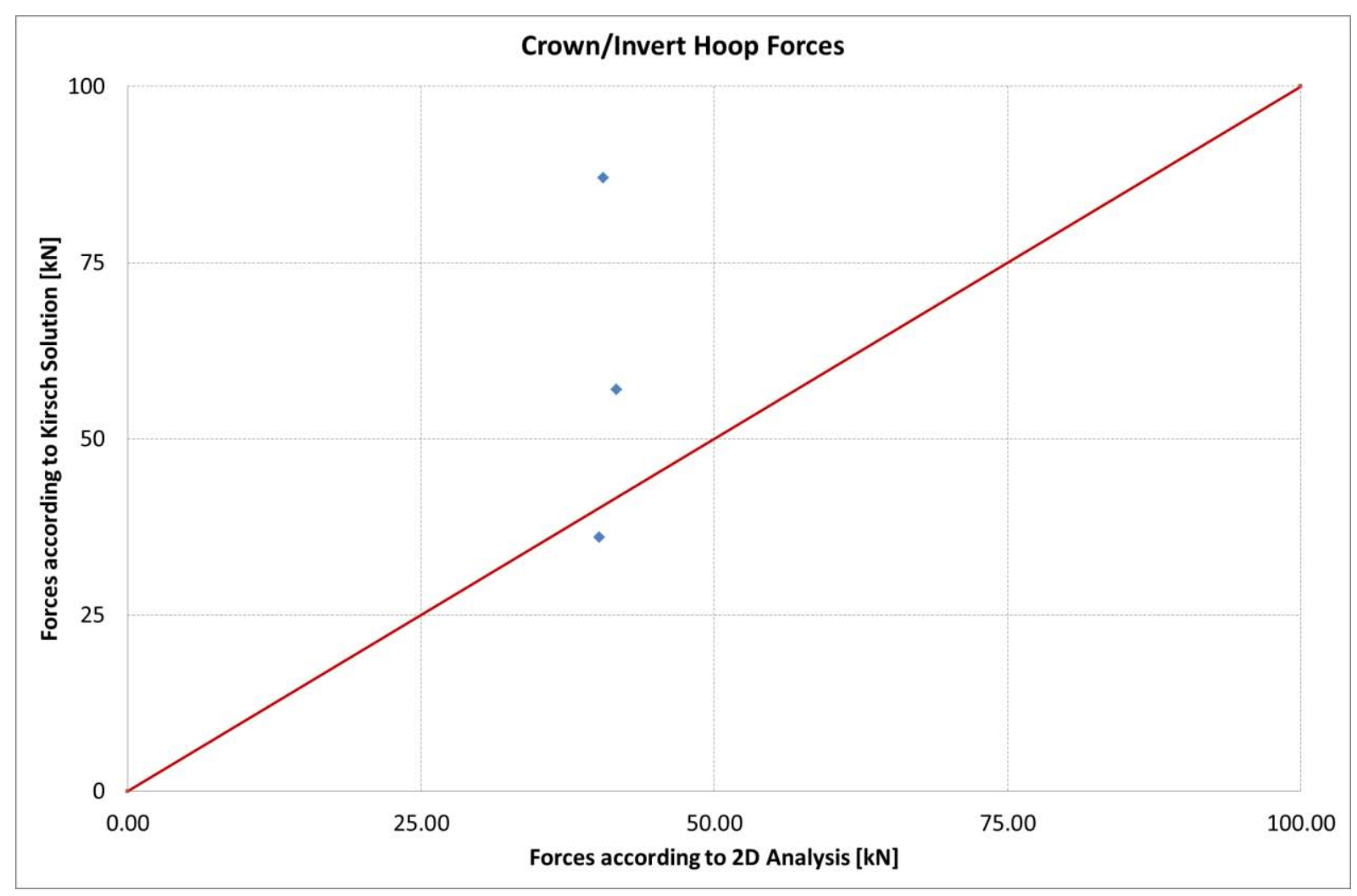

Figure 33: Comparison of 2D FE models with Kirsch solution; Hoop Forces at Crown and Invert (SF2-Cl)

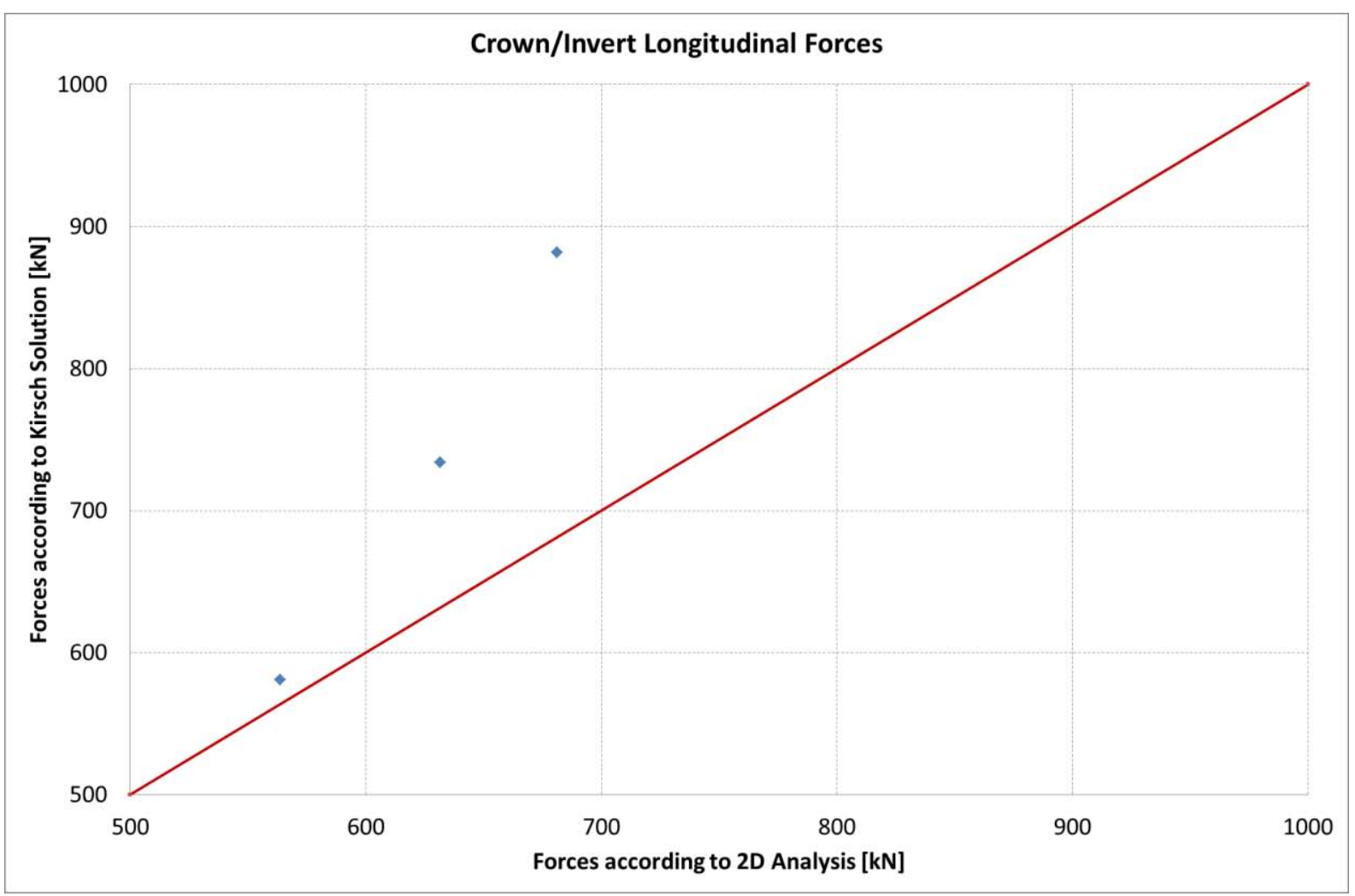

Figure 34: Comparison of 2D FE models with Kirsch solution; Longitudinal Forces at Crown and Invert (SF1-CI) 


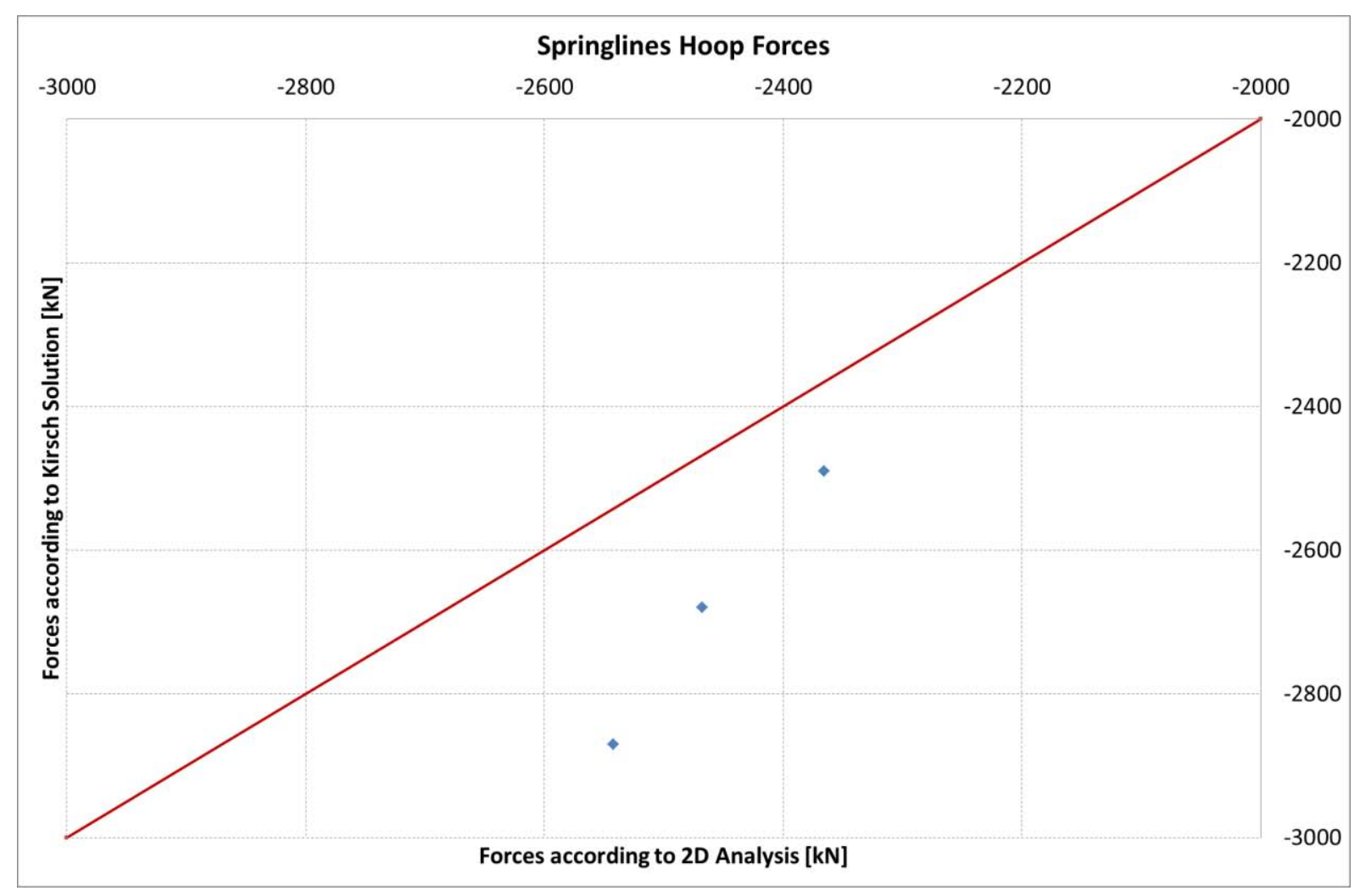

Figure 35: Comparison of 2D FE models with Kirsch solution; Hoop Forces at Springlines (SF2-SP)

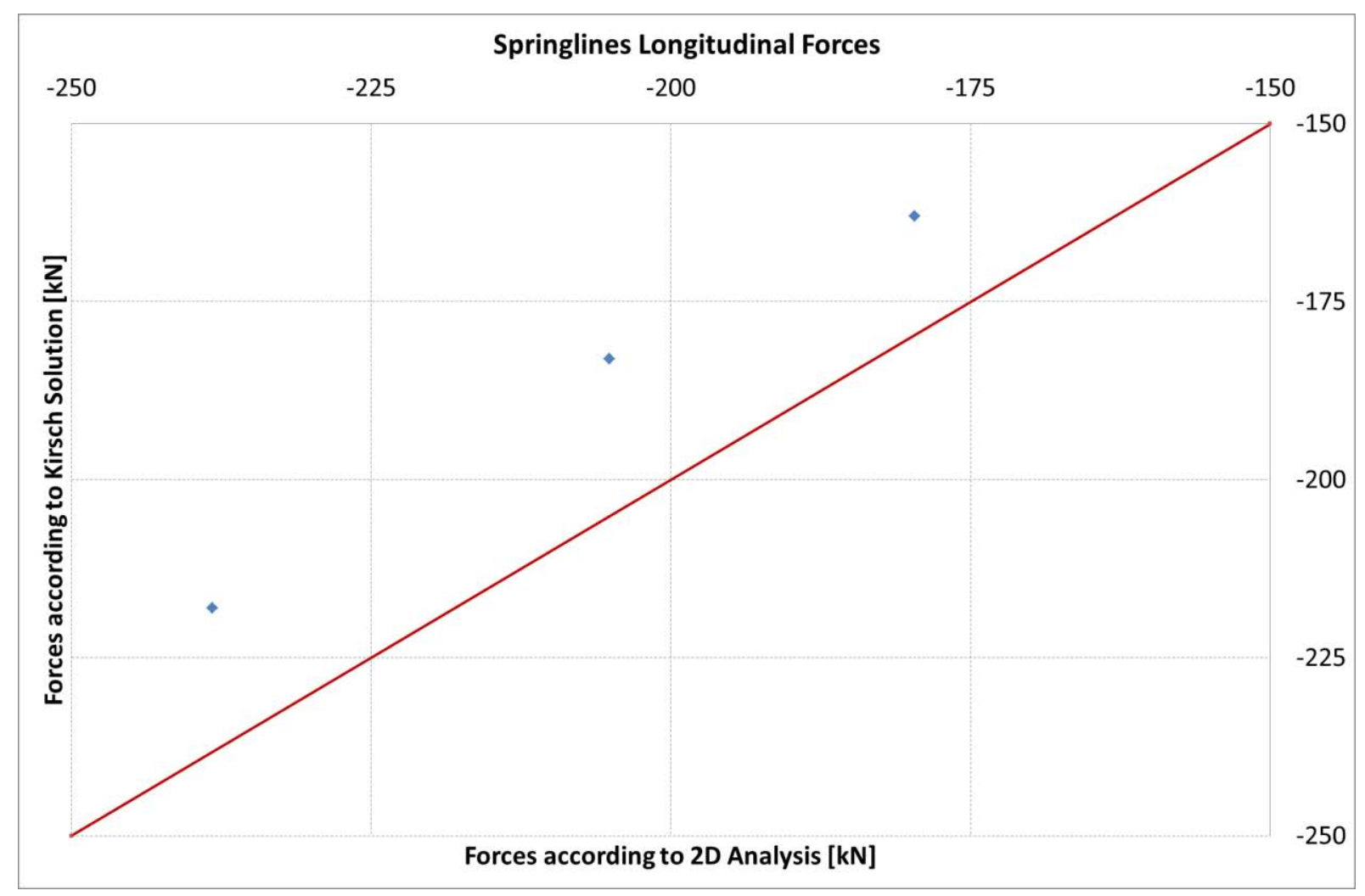

Figure 36: Comparison of 2D FE models with Kirsch solution; Longitudinal Forces at Springlines (SF1-SP) 
An additional remark based on the 2D numerical analyses is that generally, the regions affected by the stress redistributions around the opening are within approximately one diameter of the openings. These need to be considered in cases where multiple openings take place in vicinity. Furthermore, mentioning the obvious, the Kirsch solution assumes an infinite plate, whereas the $2 \mathrm{D}$ developed models have certain boundary conditions which as well affect the results, to smaller or greater extend.

\subsubsection{Interpretation and behavior in 3D}

The deformed shapes for three different models in Figure 37 - Figure 42 come to verify the concepts describing the tunnels' response in the locations of the lateral openings. The crown and invert areas behave as flat arches / beams in the longitudinal directions (B-B in Figure 7) with a maximum deflection at the midpoint, corresponding to the maximum longitudinal moment. The same location poses the maximum deflection for the cantilever idealized system in the hoop direction (A-A in Figure 7). Furthermore, the springlines behave as an arch squeezed toward the extrados $\left(A^{\prime}-A^{\prime}\right.$ in Figure 7$)$ where the maximum springline hoop moment is generated, while again a cantilever idealized stripe ( $B^{\prime}-B^{\prime}$ in Figure 7$)$ bears the maximum longitudinal moment at the springlines. 


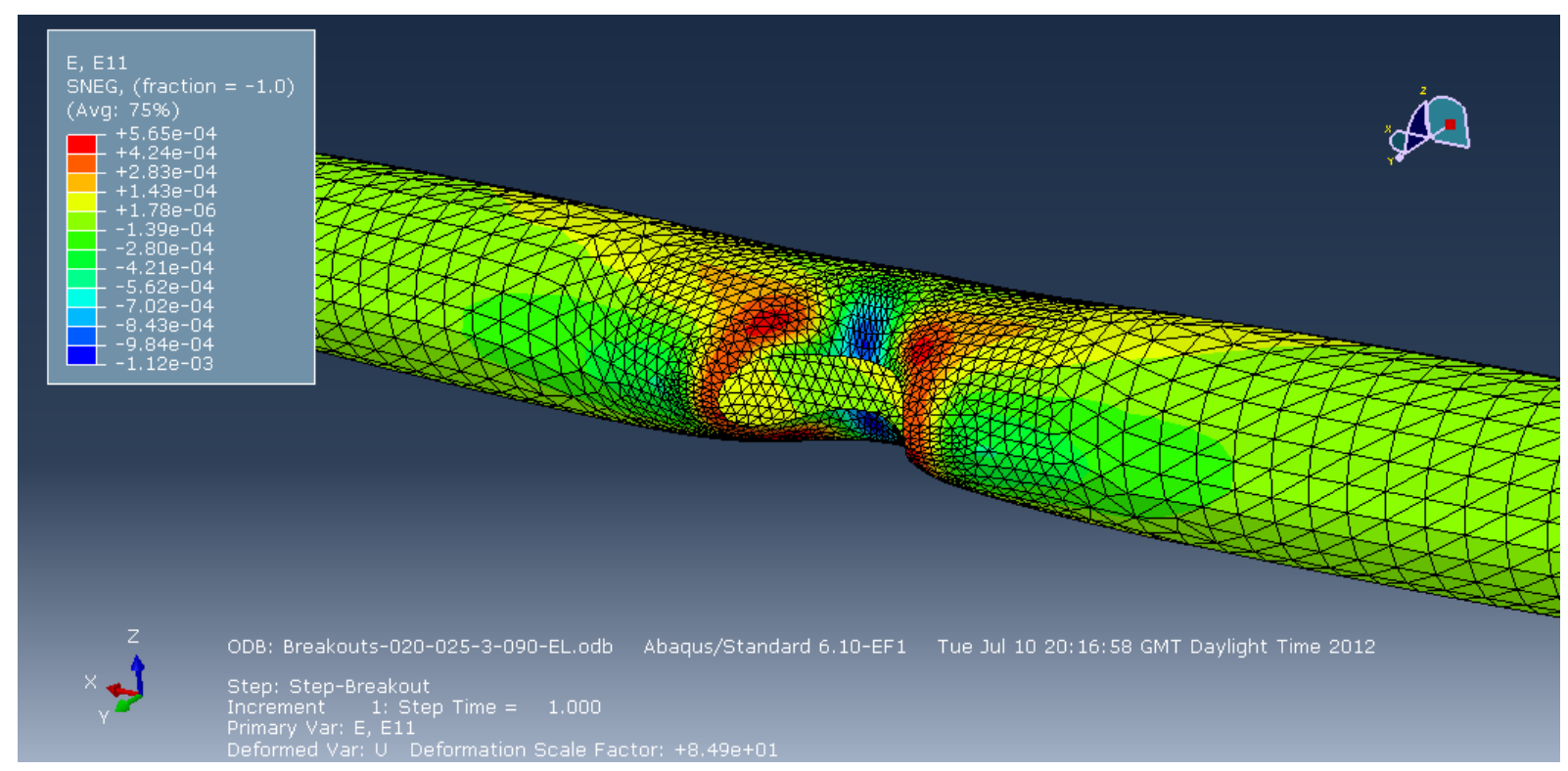

Figure 37: Model Nr.1: 3D-25-35-090; E11: Deformed shape and strains in longitudinal direction

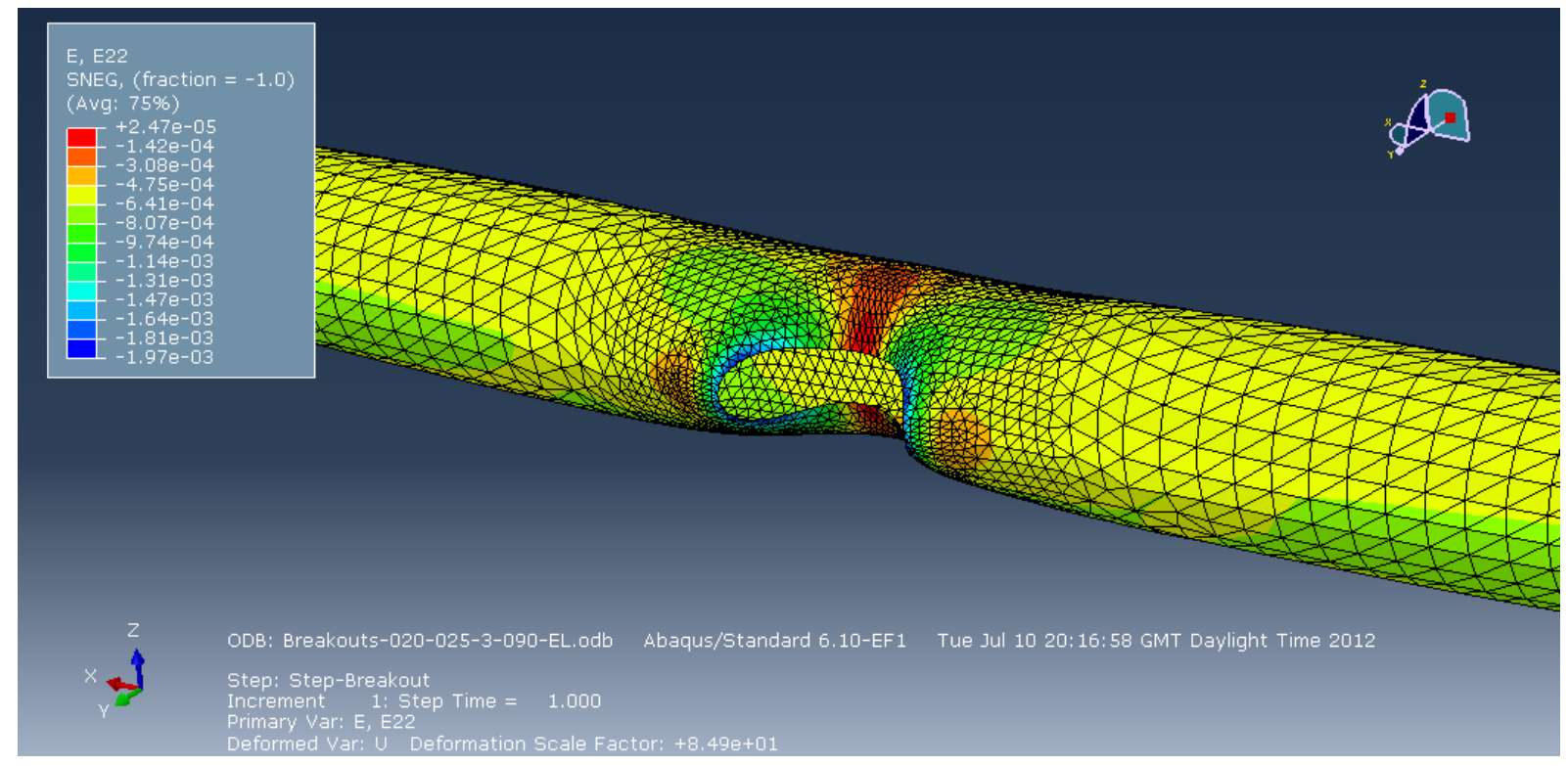

Figure 38: Model Nr.1: 3D-25-35-090; E22: Deformed shape and strains in hoop direction 


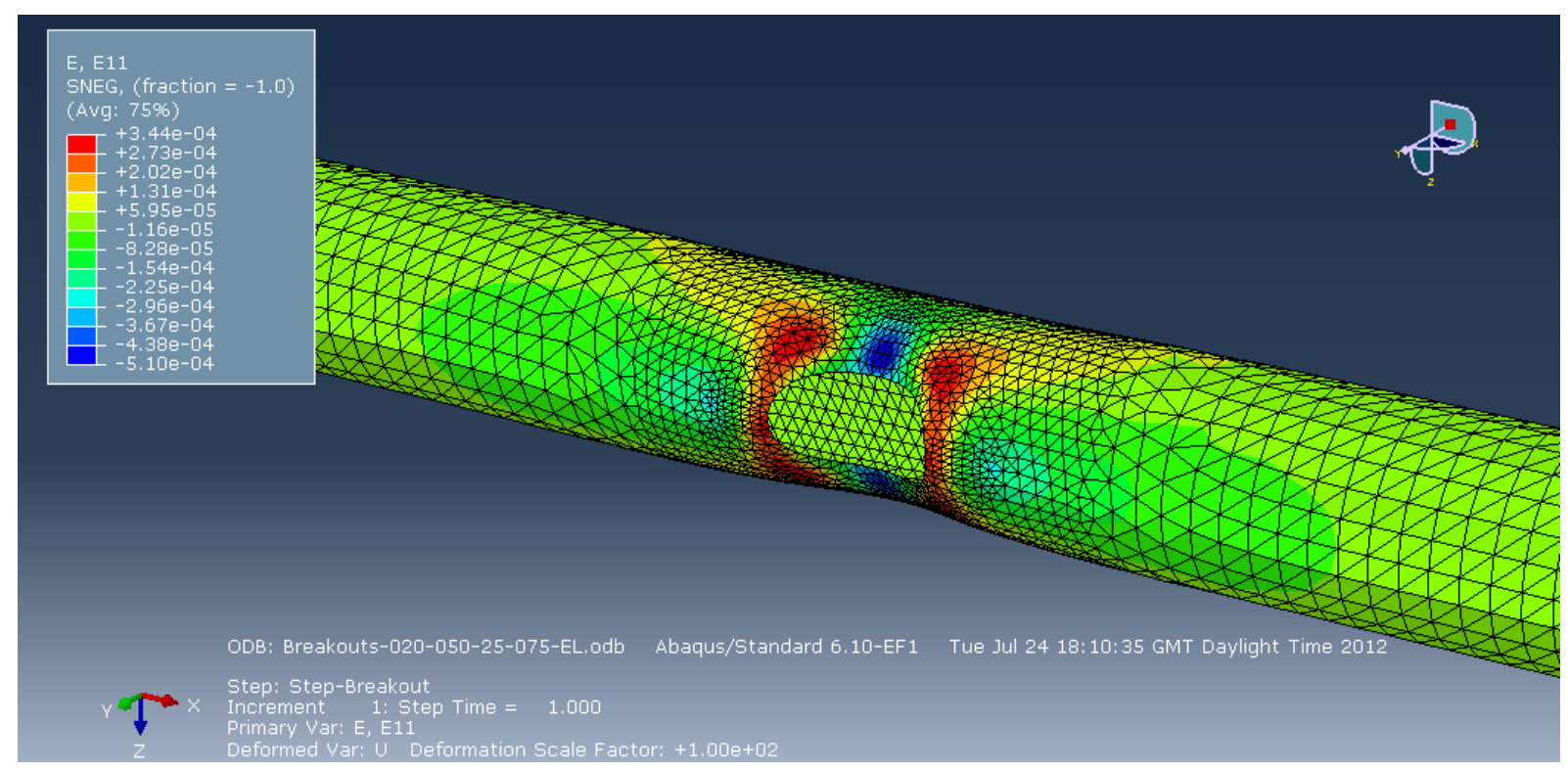

Figure 39: Model Nr.20: 3D-50-25-075; E11: Deformed shape and strains in longitudinal direction

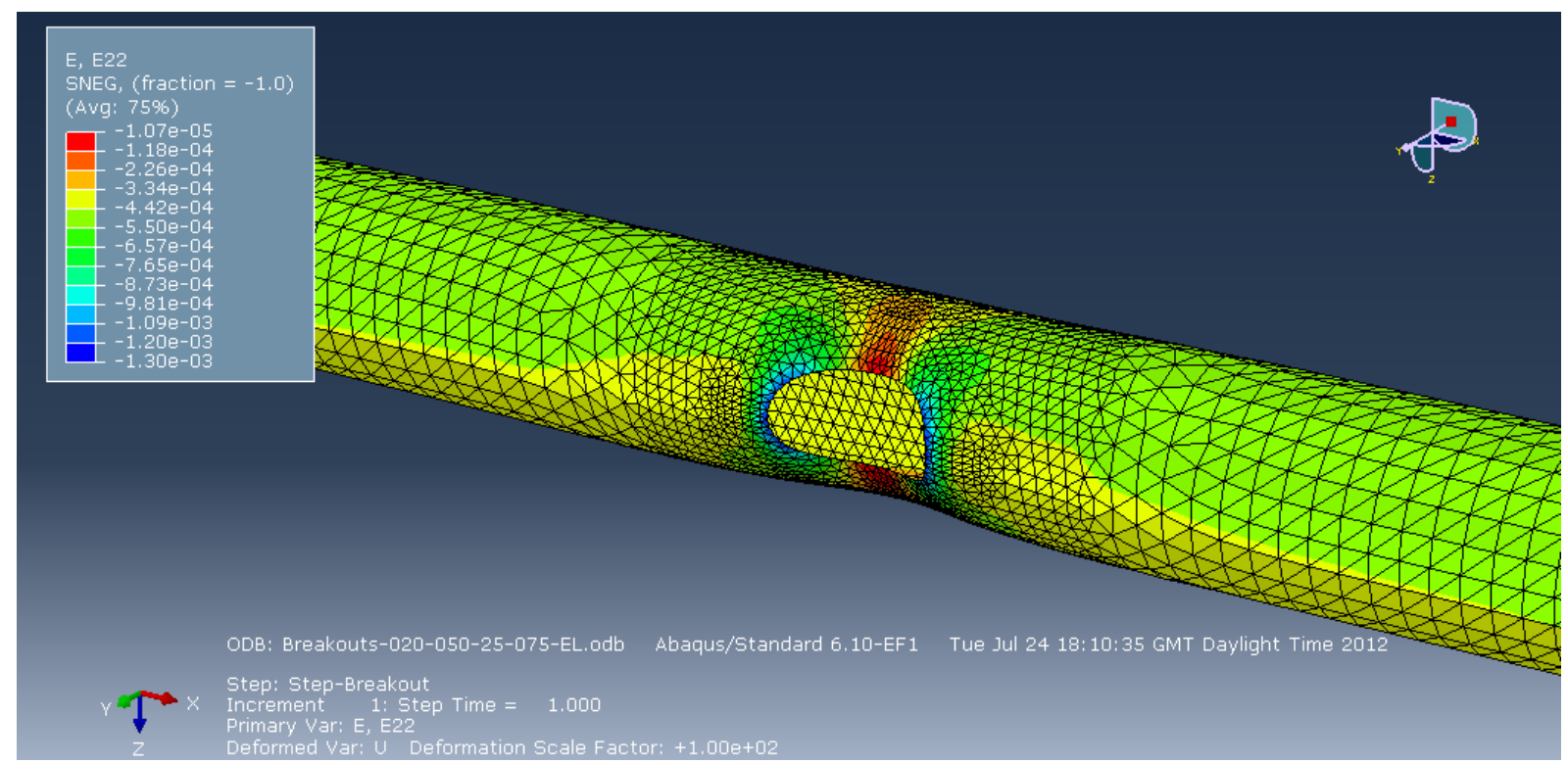

Figure 40: Model Nr.20: 3D-50-25-075; E22: Deformed shape and strains in hoop direction 


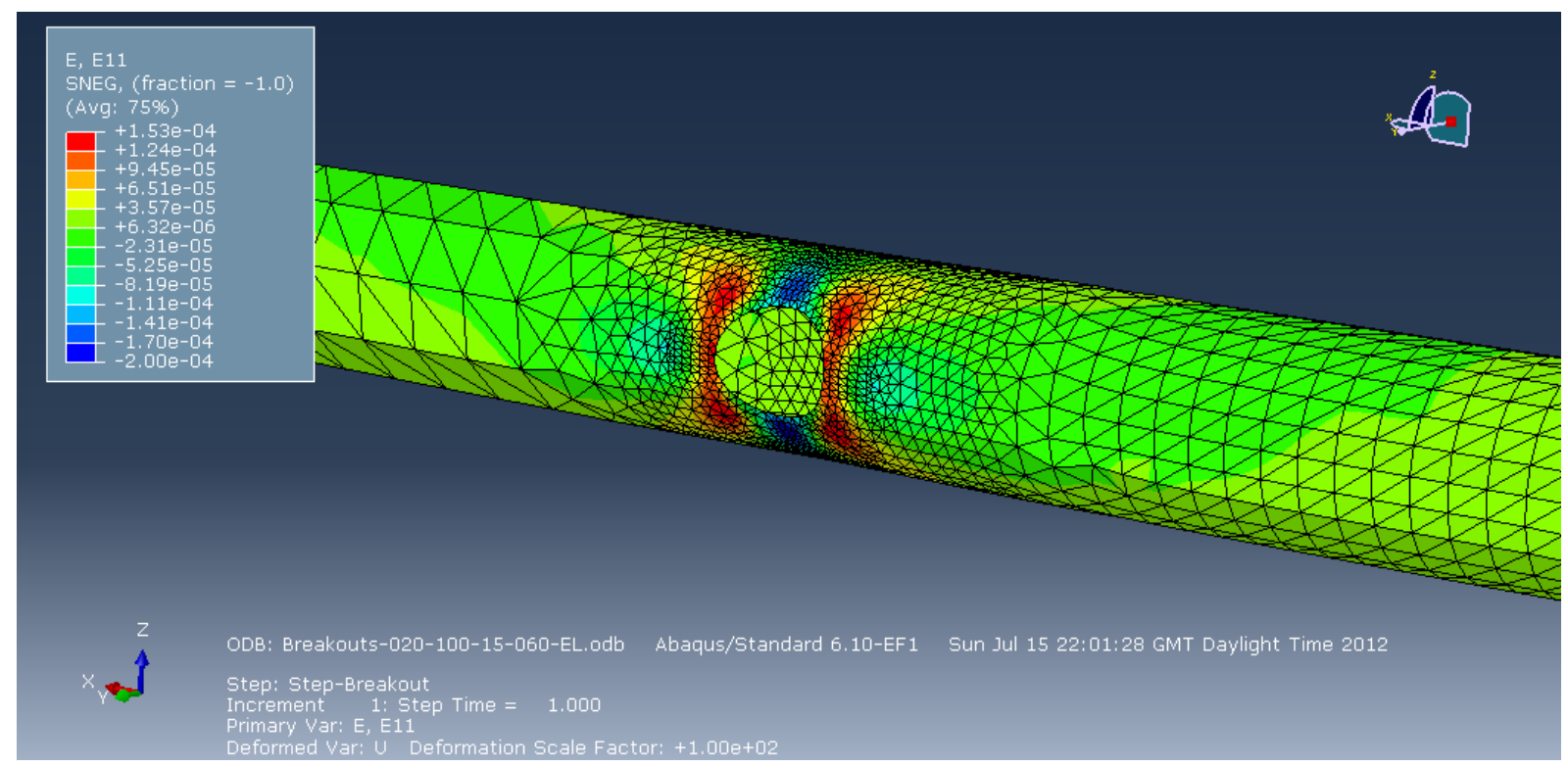

Figure 41: Model Nr.39: 3D-100-15-060; E11: Deformed shape and strains in longitudinal direction

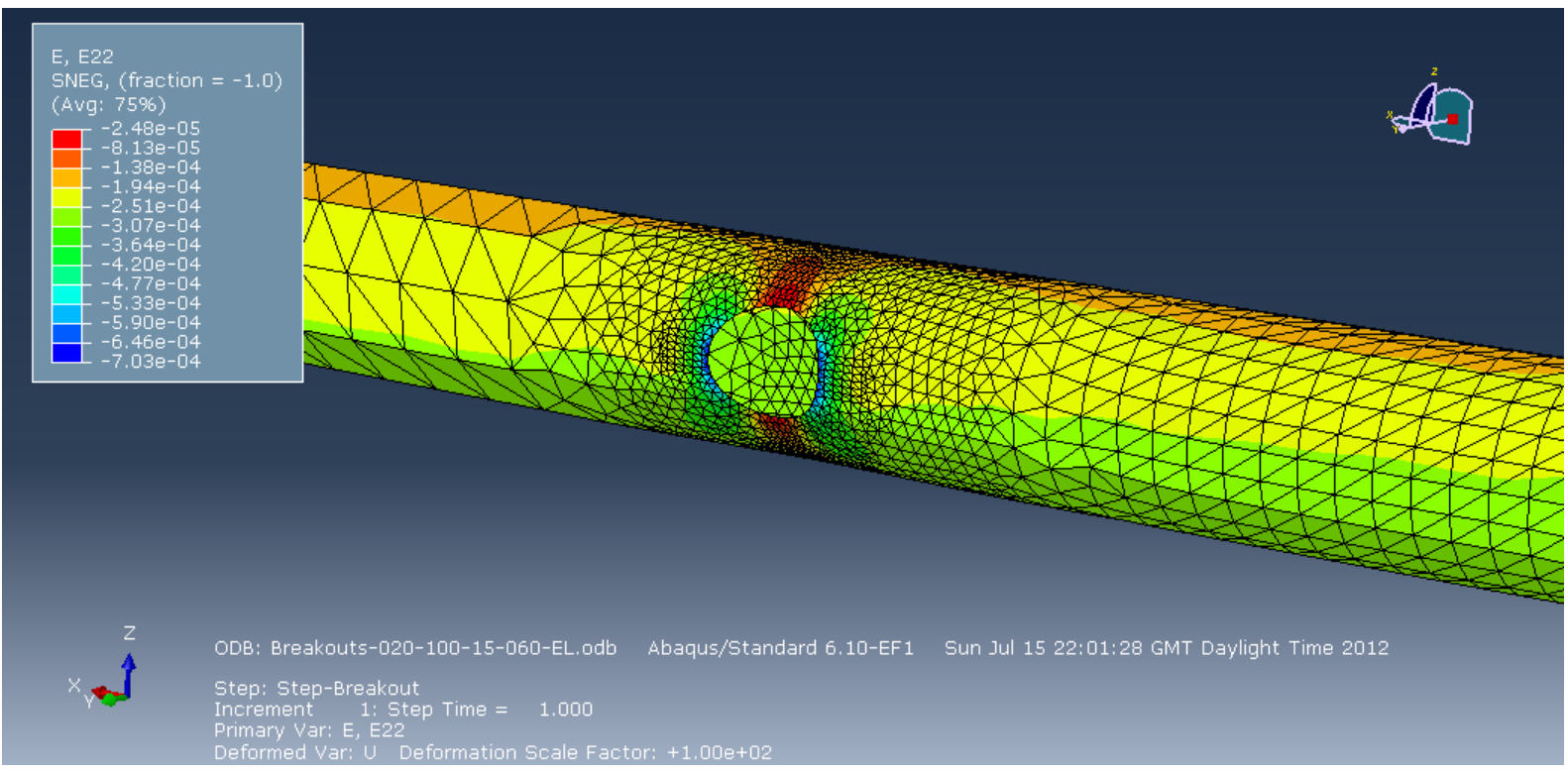

Figure 42: Model Nr.39: 3D-100-15-060; E22: Deformed shape and strains in hoop direction 
In addition, the non-deformed shapes of the structures with absolute displacement magnitudes are given in Figure 43 - Figure 45 to put them in a rational aspect. The term "relative" stands to explain that these displacements show only the additional deformation occurring due to the breakouts, after the original loading of in the tunnels (the ring closure). That is, the noted displacements in Figure 43 - Figure 45 are the differences of the initial deformations of the tunnels due to the overburden loading from the final displacements after the openings are realized (reaching $30 \mathrm{~mm}, 15 \mathrm{~mm}$ and $6 \mathrm{~mm}$ for the openings of $9 \mathrm{~m}$, $7.5 \mathrm{~m}$ and $6 \mathrm{~m}$ respectively).

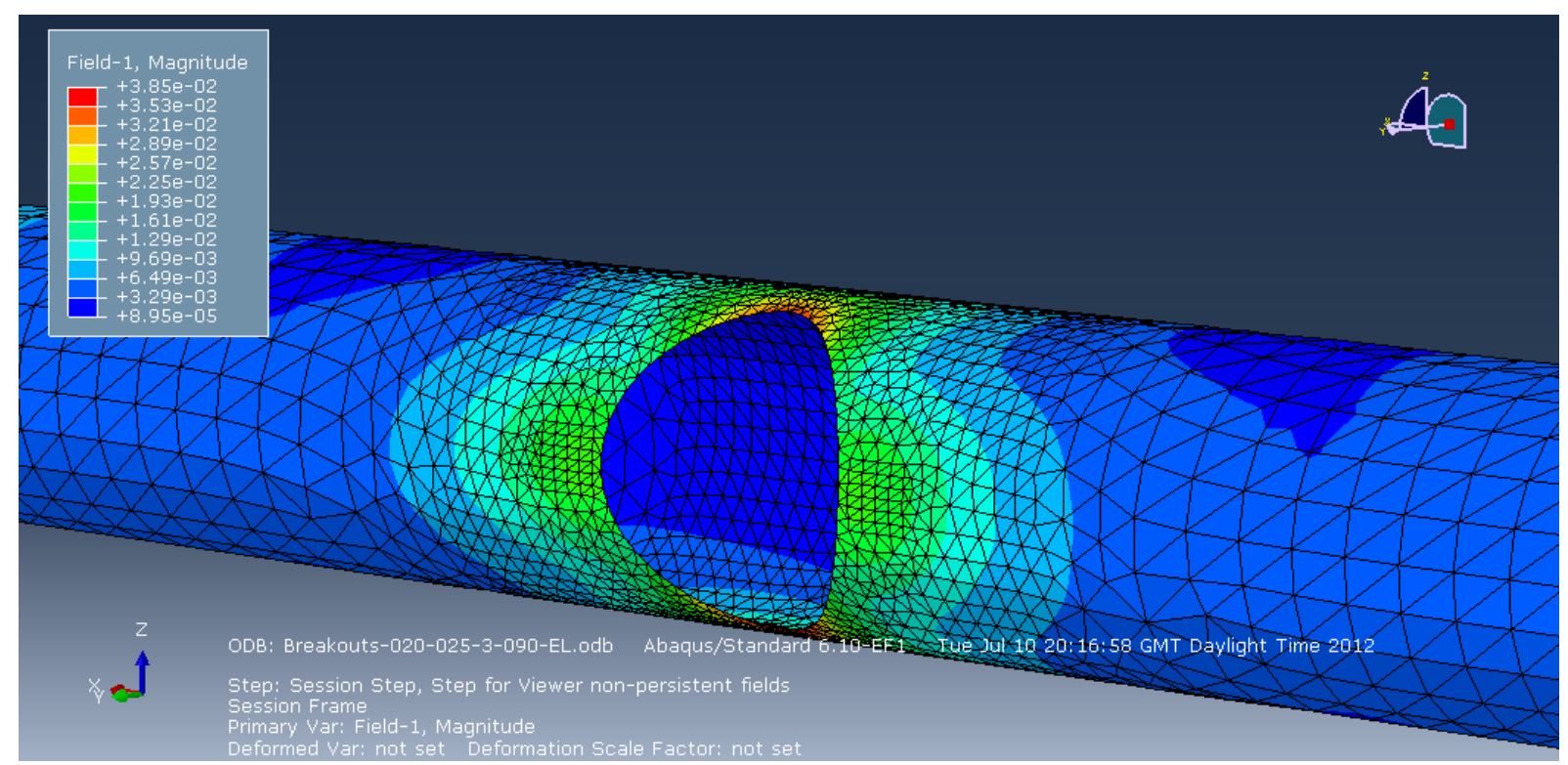

Figure 43: Model Nr.1: 3D-25-35-090; RelU: Relative absolute displacements (before and after breakout) 


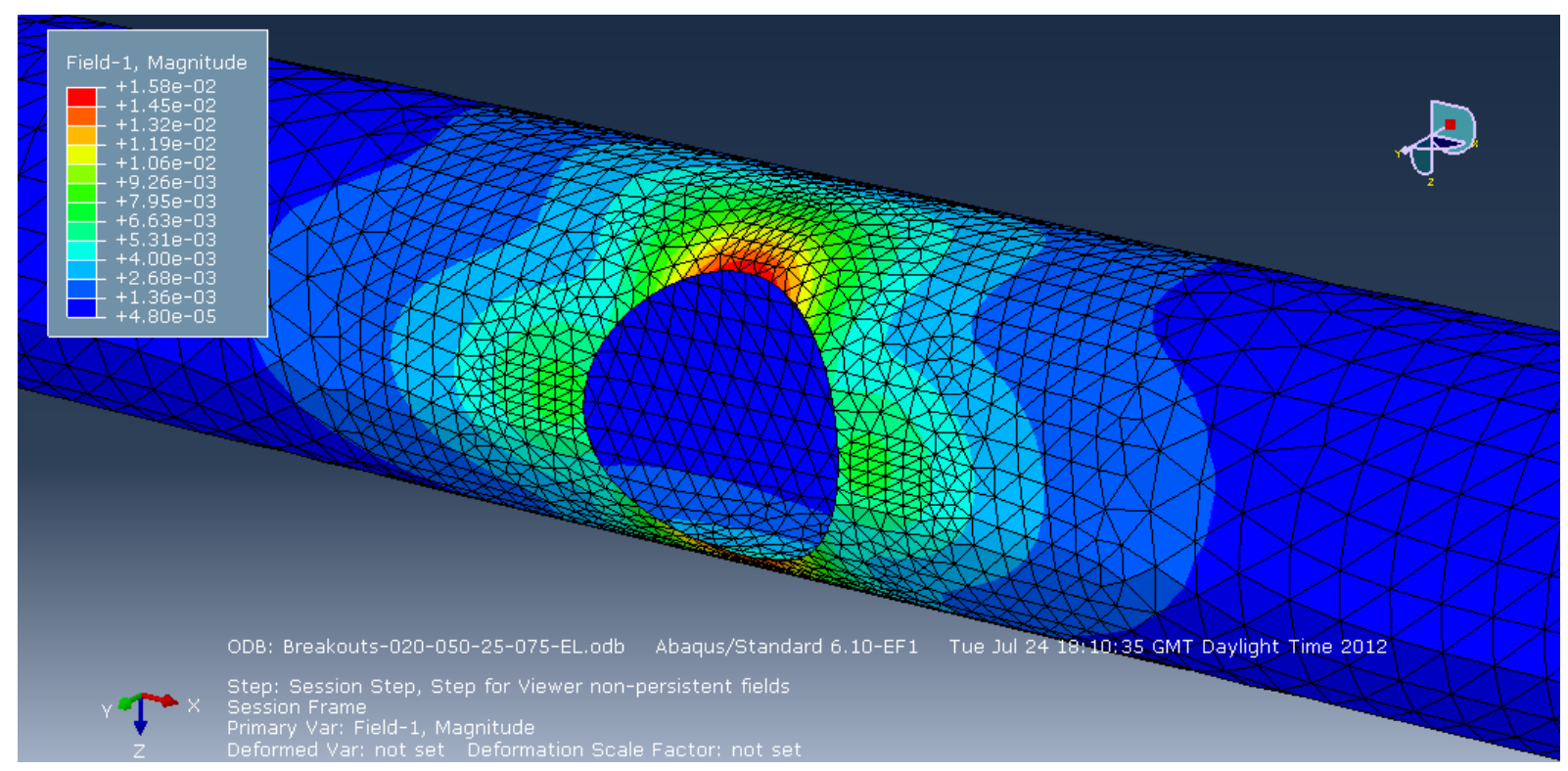

Figure 44: Model Nr.20: 3D-50-25-075; RelU: Relative absolute displacements (before and after breakout)

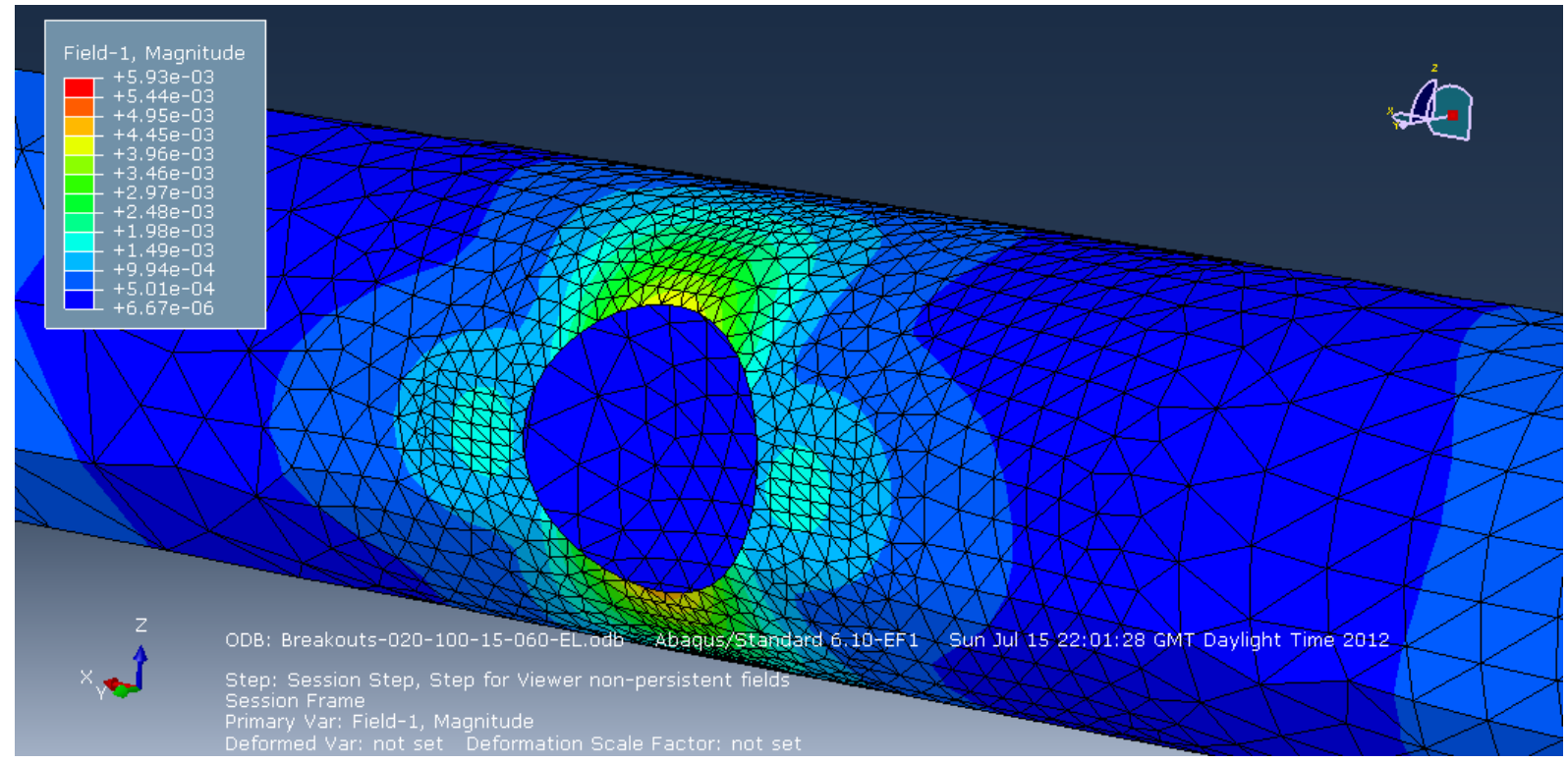

Figure 45: Model Nr.39: 3D-100-15-060; RelU: Relative absolute displacements (before and after breakout)

The graphs in Figure 49 - Figure 73 show how the normalized section forces fluctuate in dependence to varying round parameters. The values presented below are the normalized longitudinal and hoop forces (NLF, NHF) and moments (NLM, NHM) in two focus locations, i.e. Springlines (SP) and the Crown/invert $(\mathrm{Cl})$. The varied parameters have been limited to 
the soil stiffness (Young's modulus, with values 25, 50, or $100 \mathrm{MPa}$ ) and the aspect ratio of the openings (d/D with values $0.6,0.75$, and 0.9 ).

The results are not shown in relation to the depth of the tunnel, because this is inherent in the normalization of the results, i.e. the depth is analogous to the hoop force prior to breakout. This is also verified by a view of the results in Table 6, since the fluctuation of the normalized values is generally very low as the depth varies. It has to be noted that this is the case when a constant uniform stress is assumed and for sufficient depths. In the cases presented below this is not absolutely the case but this assumption is made for simplification in the representation of results. Still, some non-linearity may reside in tunnel depth as an influencing parameter.

Understandably, stiffness of the surrounding medium (soil) is a combination of the material stiffness and the geometry, so for shallow tunnels, the confinement is less dependent on the soil Young's modulus and more dependent on the overburden (i.e. the depth of the solid body constraining the tunnel). Note the confinement in the present cases is rather dependent on the flexural stiffness of the overburden (the soil forms a "bridge" above the tunnel), so it should be understood as somewhat proportional to the $3^{\text {rd }}$ power of the depth from the surface to the tunnel crown. The confinement is also dependent on the stress state of the material surrounding the tunnel, including the lateral pressure coefficient $\left(K_{0}\right)$, but this effect was deliberately downgraded in the context of the present thesis in order to clarify other influences.

From the illustrations above it can be seen that certain longitudinal stresses develop within the lining already in the ring closure state. These are attributed to the following amalgam of reasons (from most to less relevant):

- Concrete's Poisson - effect: Based on the ratio of the longitudinal to hoop forces at ring closure from the results in the Appendix, a lateral stress coefficient of $\mu=\sigma_{y y} / \sigma_{x x}=0.156$ is calculated. This appears to represent the inherent Poisson nature of the material, i.e. the material responds with a longitudinal expansion once compressed in the hoop direction. Since the material is constrained this lateral expansion is transformed to compression. The assigned Poisson ratio of 
$v=\varepsilon_{\mathrm{yy}} / \varepsilon_{\mathrm{xx}}=0.20$ is comparable to the coefficient $\mu$ above, which supports this hypothesis.

- Model imperfection 1: The side "buffers", are indeed softer constraints but the side reaction is not totally eliminated.

- Model imperfection 2: The lining is modeled in full/fixed contact (no slip interface) with the elastic surrounding medium, which means that constraints from this bedding condition create a reaction and therefore a uniformly distributed compressive force along the periphery of the lining. Still this is not expected to be very relevant, as stronger longitudinal forces appear for softer soils (see also Table 5 and Table 6). Moreover, the additional non-linear elastic soil calculations (Mohr Coulomb) show that longitudinal compressive forces at ring closure decrease but are still substantial.

Interferences due to model imperfection " 2 " that should be expected in the results are that some of the forces that should be re-distributed within the lining are borne by the soil itself. This is most likely a non-realistic and non-conservative assumption. Nevertheless, this is an effect that takes place at a small distance from the tunnel and the soil to structure Young's moduli ratio is in $1 / 100$ or more, so the results are expected to be slightly affected due to this situation, by $1 \%$ or less.

Based on the analyses as represented in the graphs further below, the following trends can be summarized:

The longitudinal forces at crown/invert are typically tensile and decrease with increasing confinement and decreasing child tunnel size (Figure 49, Figure 50): With respect to the effects on NLF for the $\mathrm{Cl}$ area, the FE analyses show a very strong dependence on the confinement from the surrounding material, and consequently on the soil Young's modulus $\left(\mathrm{NLF} \approx 0.003 \cdot \mathrm{E}_{\mathrm{soil}}\right)$. The longitudinal forces in the $\mathrm{Cl}$ are generally expected to be tensile, based on the plate theory (Kirsch) or a simplified strut-and-tie model. For larger openings ( $d / D=0.9$ ) and greater overburdens, tension appears to diminish, but in fact it is transferred to other areas of the lining (Figure 46). These areas lie further in a diagonal direction of approximately 45 degrees and this phenomenon overrides the effects of confinement, i.e. 
soil stiffness does not appear to have an effect in these cases, perhaps implying that the effect does not obey any soil-structure interaction response. The child/parent aspect ratio also seems to have an influence on the CI NLF results, with an inverse linear proportionality in smaller child tunnels and non-linear analogy for larger openings (e.g. d/D>0.75).

The longitudinal forces at springlines are compressive and they tend to decrease with increasing soil stiffness and child tunnel size (Figure 51 - Figure 52): The higher the confinement around the tunnel the lower the NLF at the sides of the openings. However this trend appears to obtain an asymptotic cease toward higher soil stiffness. Of course the inherent longitudinal compression needs to be considered which may lead to interferences in this phenomenon. This compression adds up to the existing longitudinal compression which has been mainly attributed to the Poisson effects. In all cases, the forces appear to be compressive yet not high enough to pose substantial concerns with respect to the design (e.g. need for thickening), based on this series of analyses. NLF at SP are consistently dependent on both the child tunnel relative diameter and the Young's modulus of the soil, inversely proportional in both cases, i.e. the stresses get lower for larger diameters and higher $E_{\text {soil }}$ values.

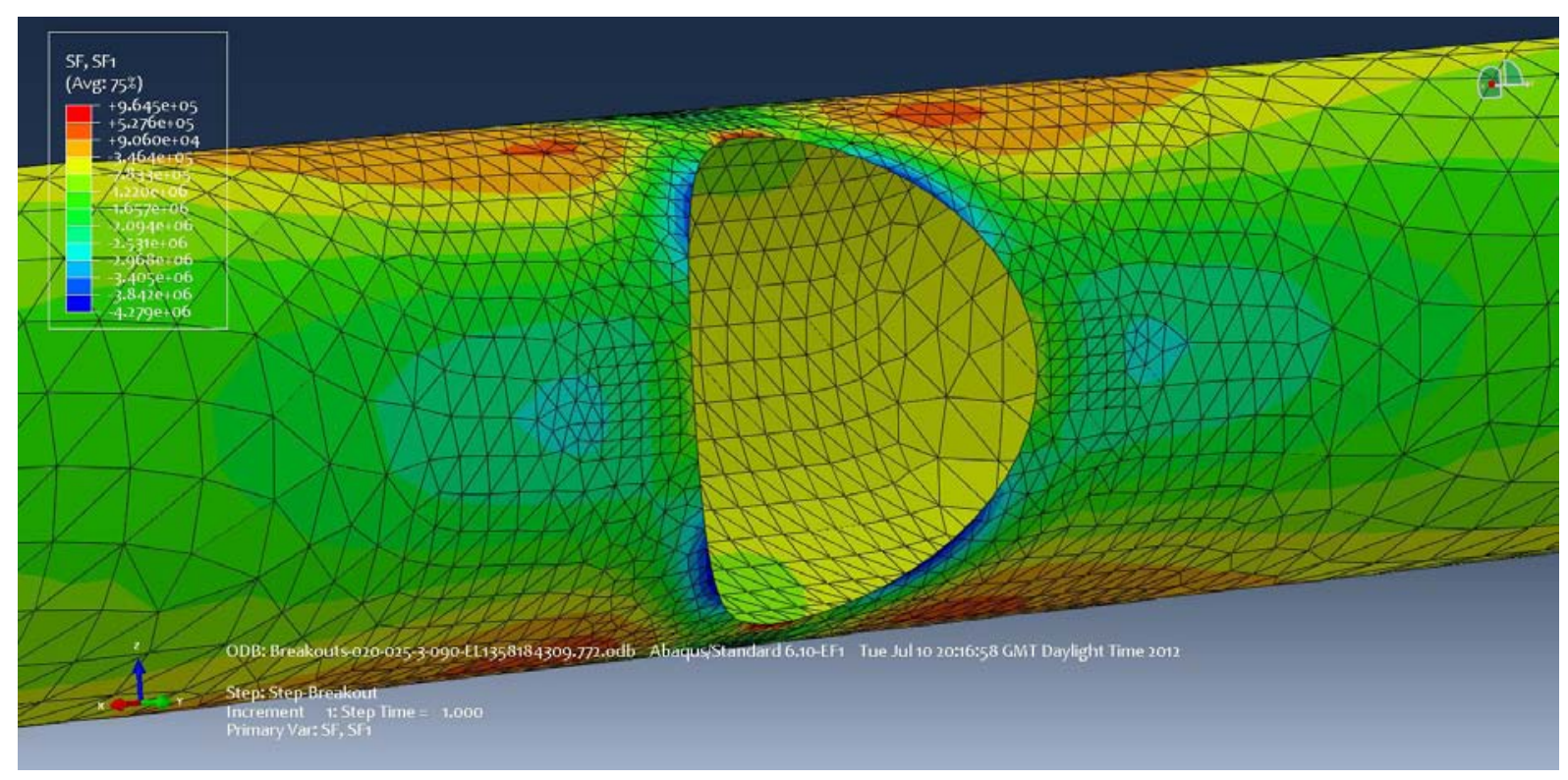

Figure 46: Model Nr.1: 3D-25-35-090; SF1: Longitudinal forces 


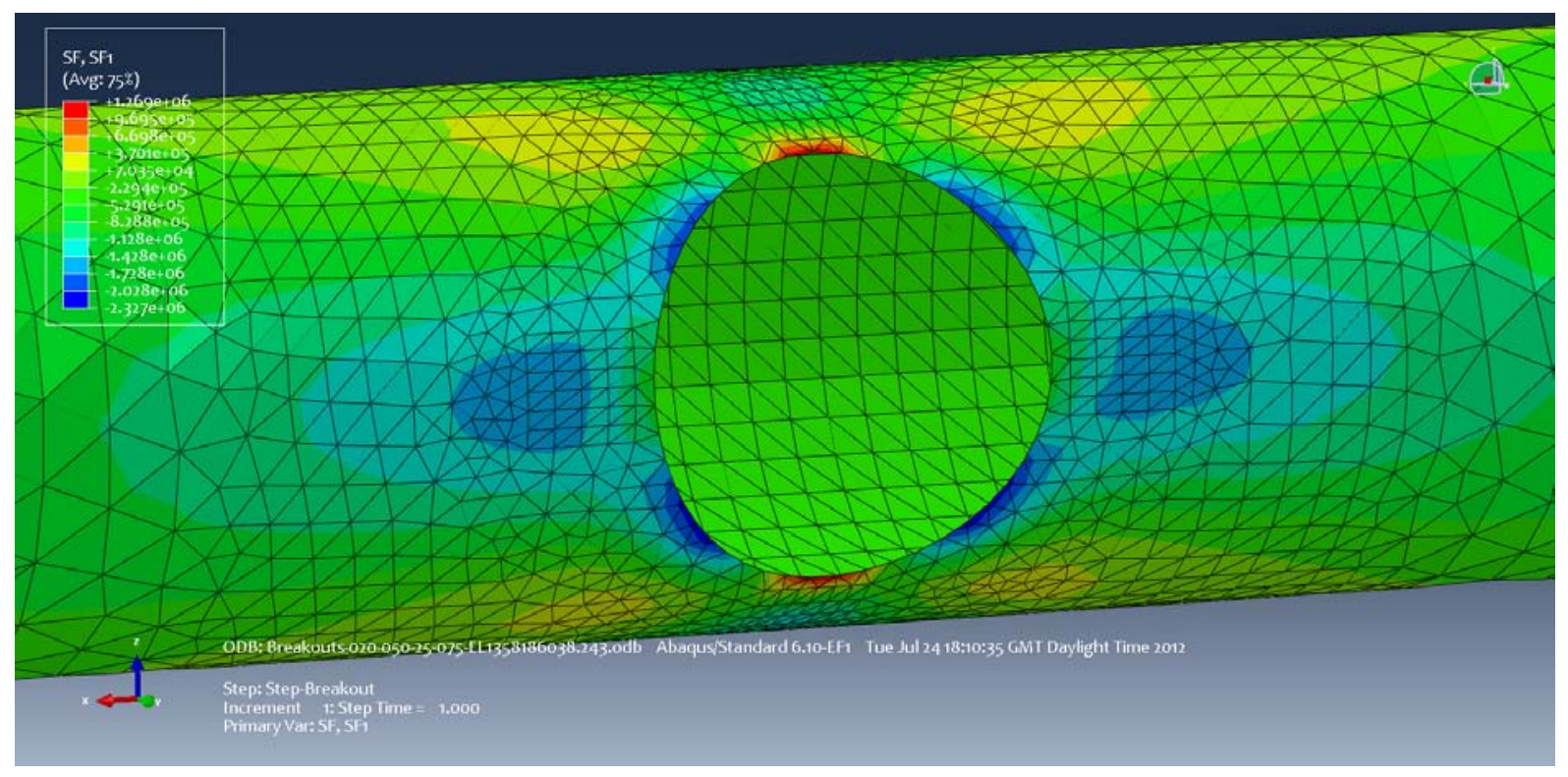

Figure 47: Model Nr.20: 3D-50-25-075; SF1: Longitudinal forces

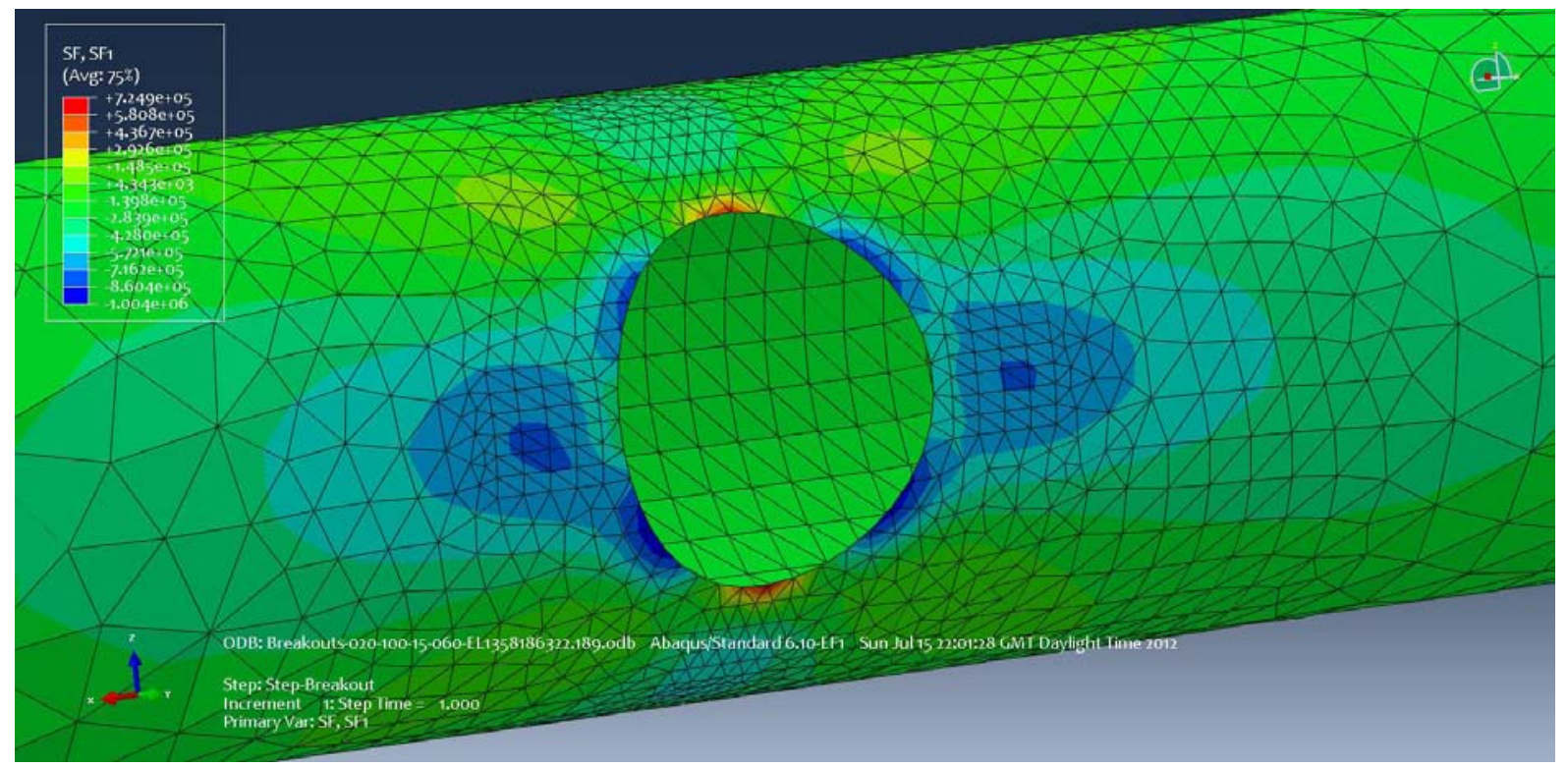

Figure 48: Model Nr.39: 3D-100-15-060; SF1: Longitudinal forces 


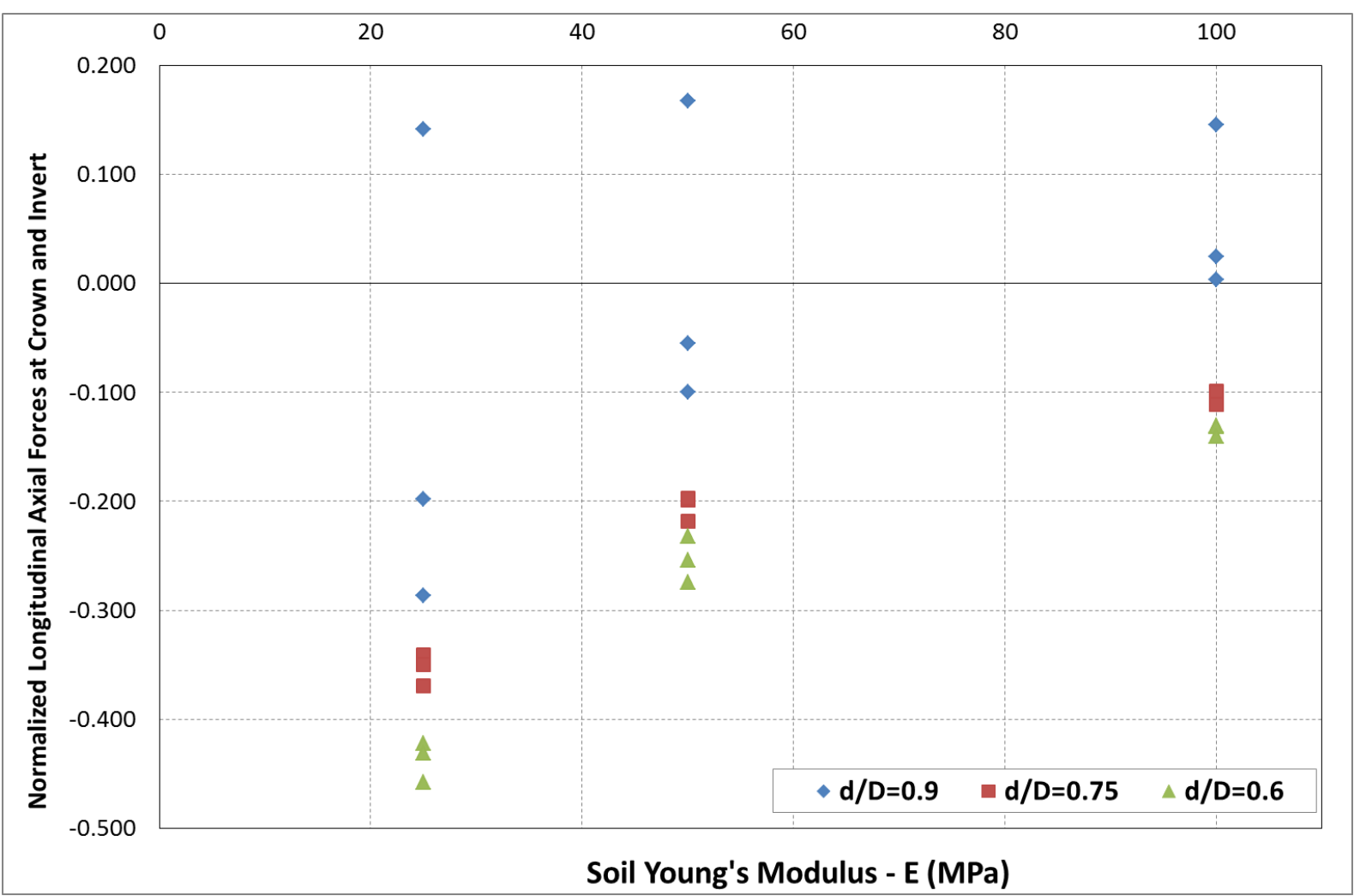

Figure 49: Influence of soil Young's modulus on Longitudinal Forces at Crown/Invert (sf1-Cl-norm)

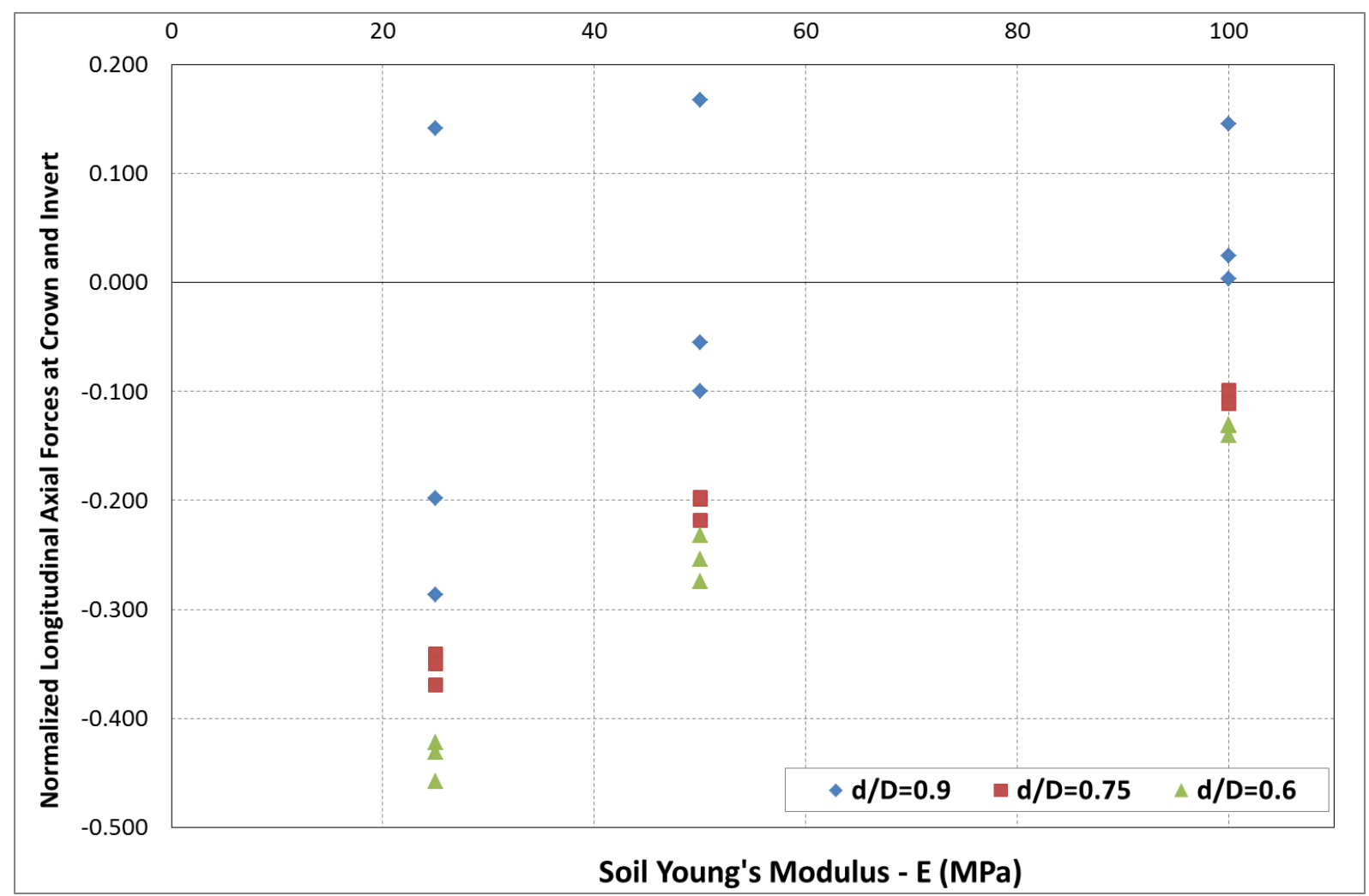

Figure 50: Influence of child-parent tunnel aspect ratio on Longitudinal Forces at Crown/Invert (sf1-Cl-norm) 


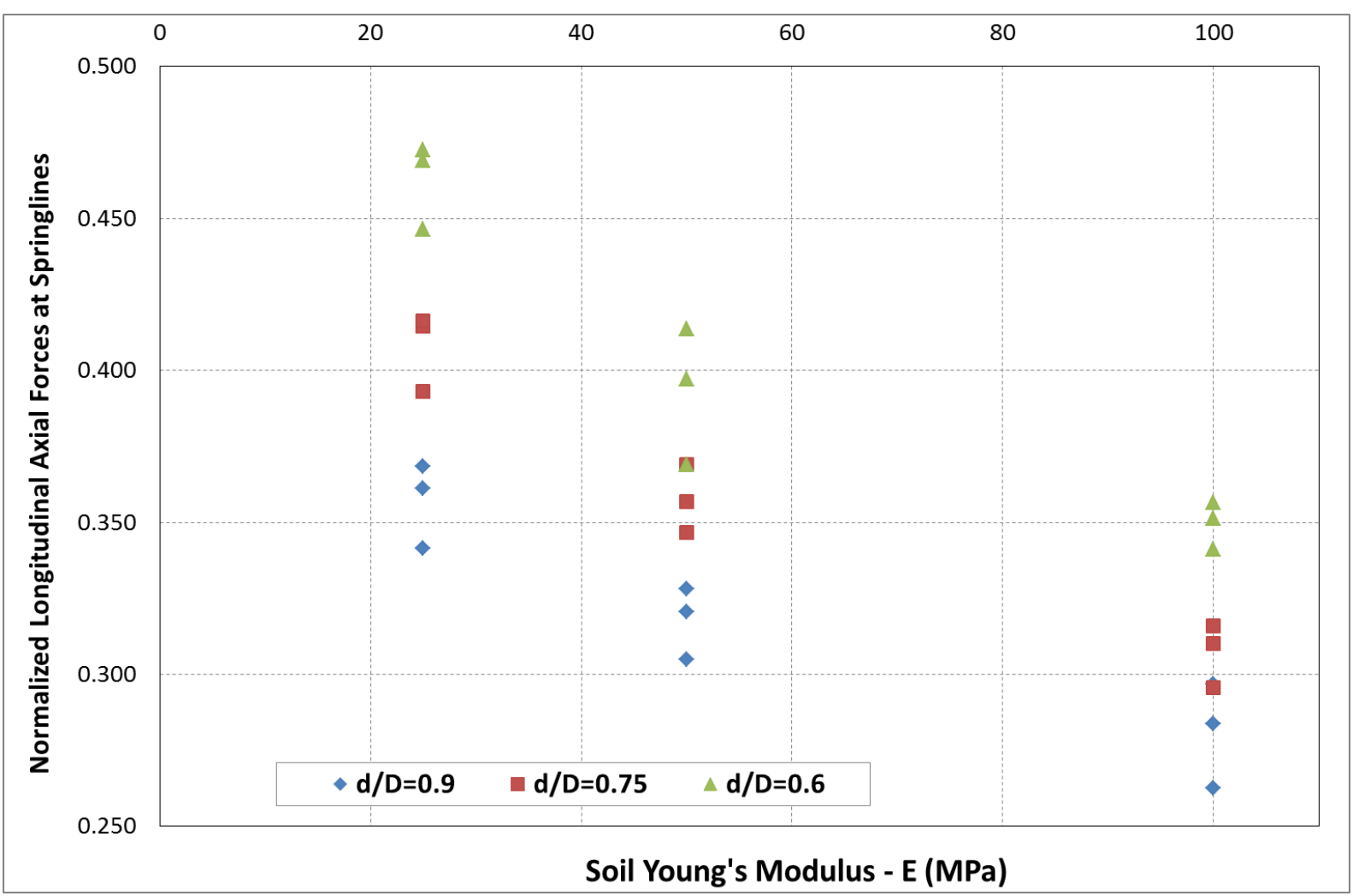

Figure 51: Influence of soil Young's modulus on Longitudinal Forces at Springlines (sf1-SP-norm)

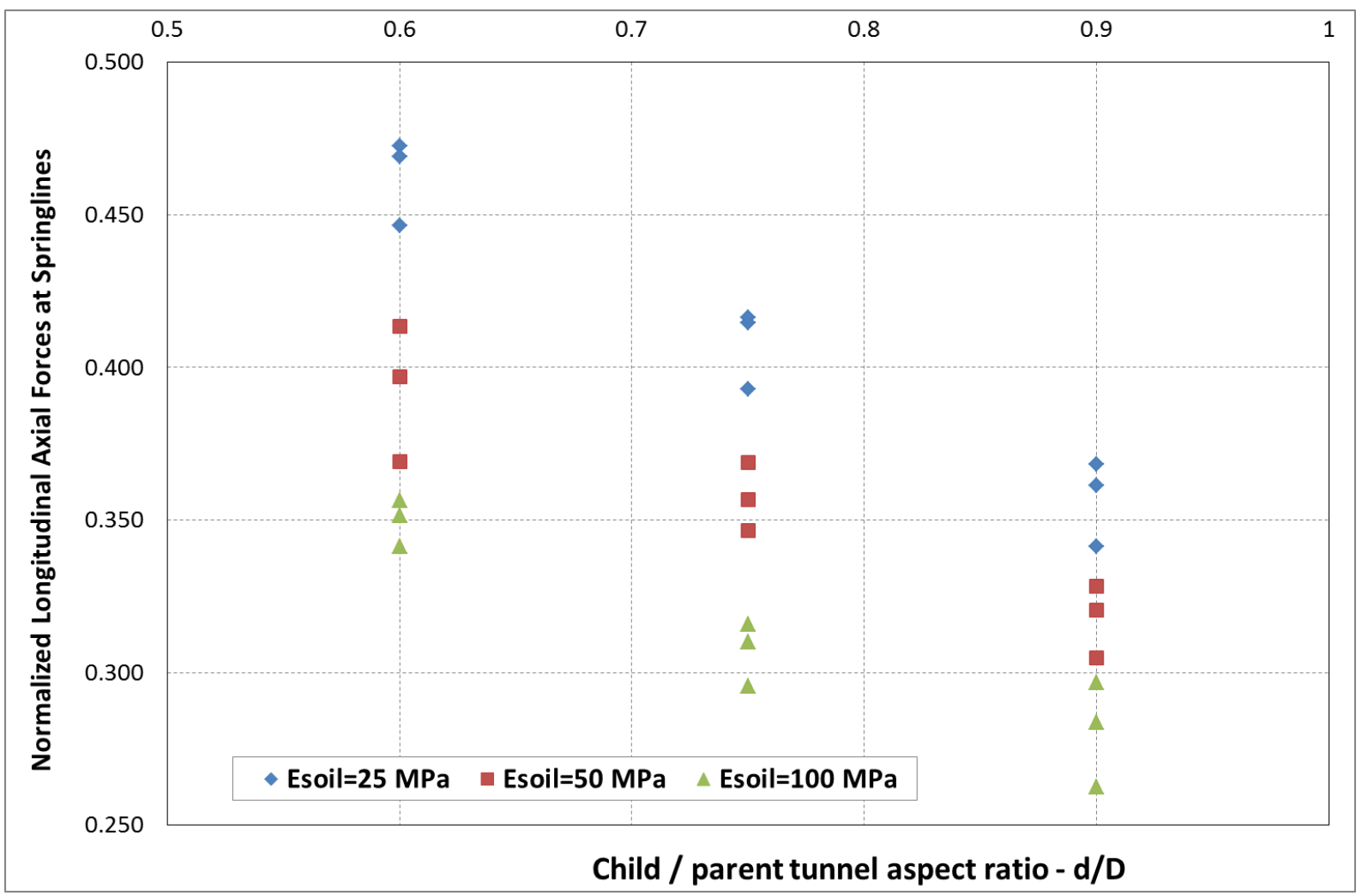

Figure 52: Influence of child-parent tunnel aspect ratio on Longitudinal Forces at Springlines (sf1-SP-norm) 
The hoop forces at crown/invert are rather insensitive to the varying paparameters (Figure 56 - Figure 57): No particular sensitivity appears in the hoop forces at $\mathrm{Cl}$. As plotted, they are consistent with each other, i.e. an average NHF of 0.21 of the hoop force before breakout with a coefficient of variation of $8 \%$ for all 3D analyzed cases. For large child diameters and low soil stiffness, this scatter slightly increases to $10 \%$. This generally low scatter of results means that the hoop forces at $\mathrm{Cl}$ for the investigated structures are simply a fraction of the original hoop forces, with low influence on other factors, or in other wording that the hoop force concentration at $\mathrm{Cl}$ is rather a membrane effect. In all cases the amount of hoop force concentrated at these locations is a small fraction of what is expected at ring closure and along the entire tunnel span, so no particular measure should be anticipated to receive this stress re-distribution. Still it should be noted that the loss of compressive state will have an impact on the area's bending resistance.

The hoop forces at springlines reflect a high concentration of compression (Figure 58 Figure 59): The hoop force concentration at the sides lies within the range of 2.5 to 3 times the original hoop forces before the breakout. This hoop compression concentration is strongly dependent on the soil Young's modulus; however it shouldn't be expected to diminish for very strong soil stiffness (e.g. non-squeezing rock). The influence of the breakout's size (d/D) does not seem to have a relevant influence on the results. It is noted that this very high compression should be one of the main design drives, i.e. thickening at the sides is most probably indicated by the design to transfer the higher hoop loading. 


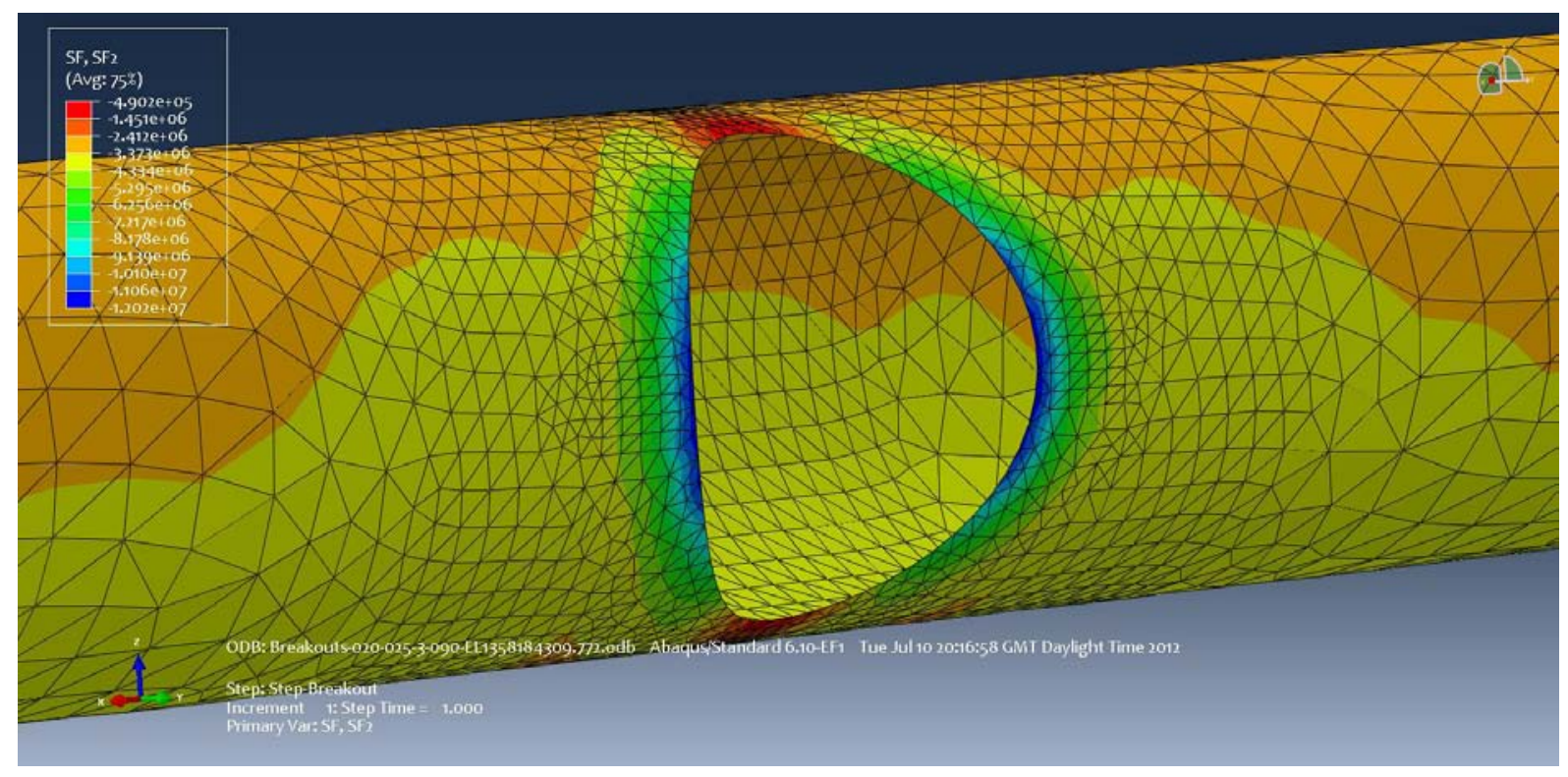

Figure 53: Model Nr.1: 3D-25-35-090; SF2: Hoop forces

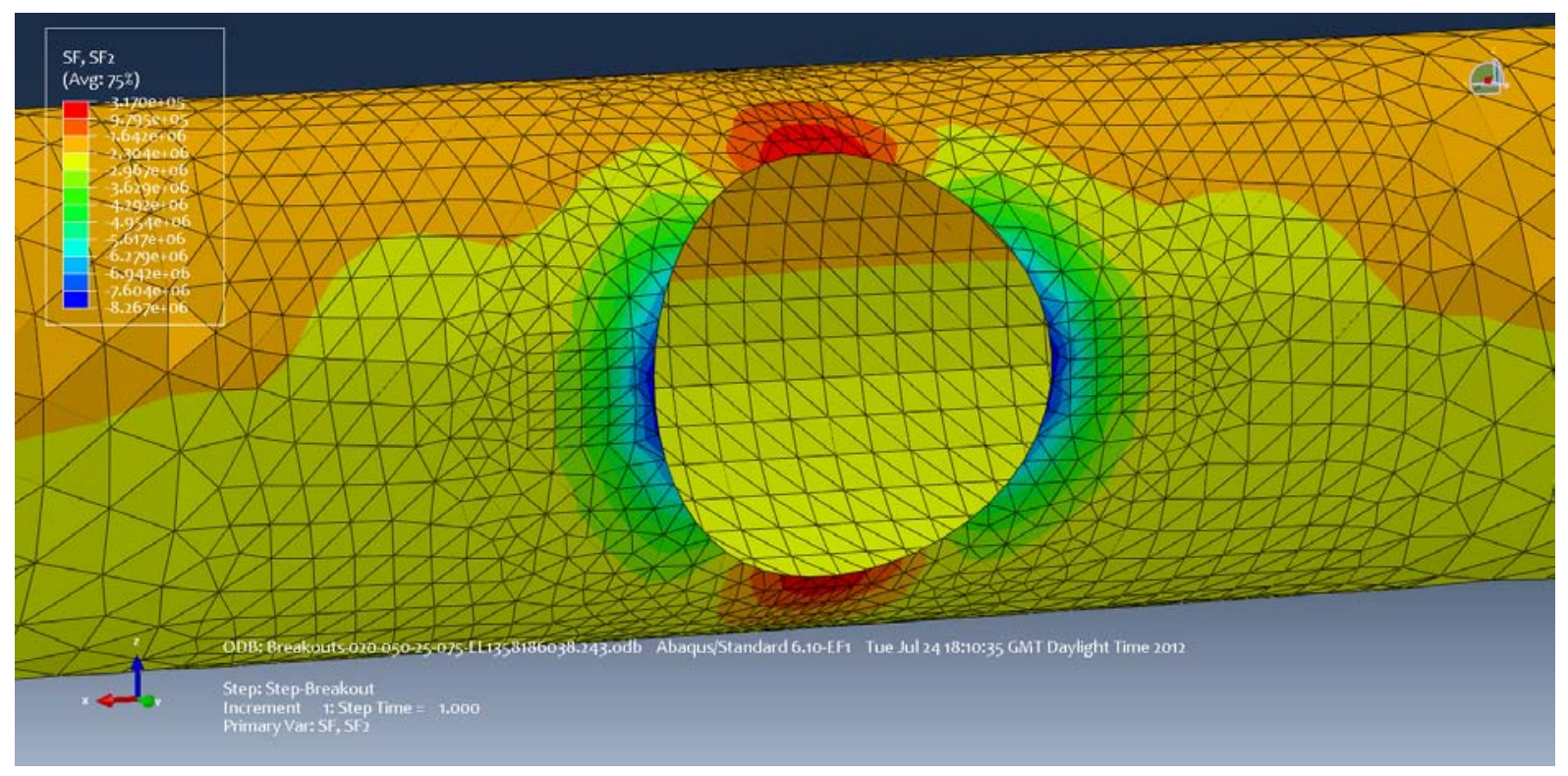

Figure 54: Model Nr.20: 3D-50-25-075; SF2: Hoop forces 


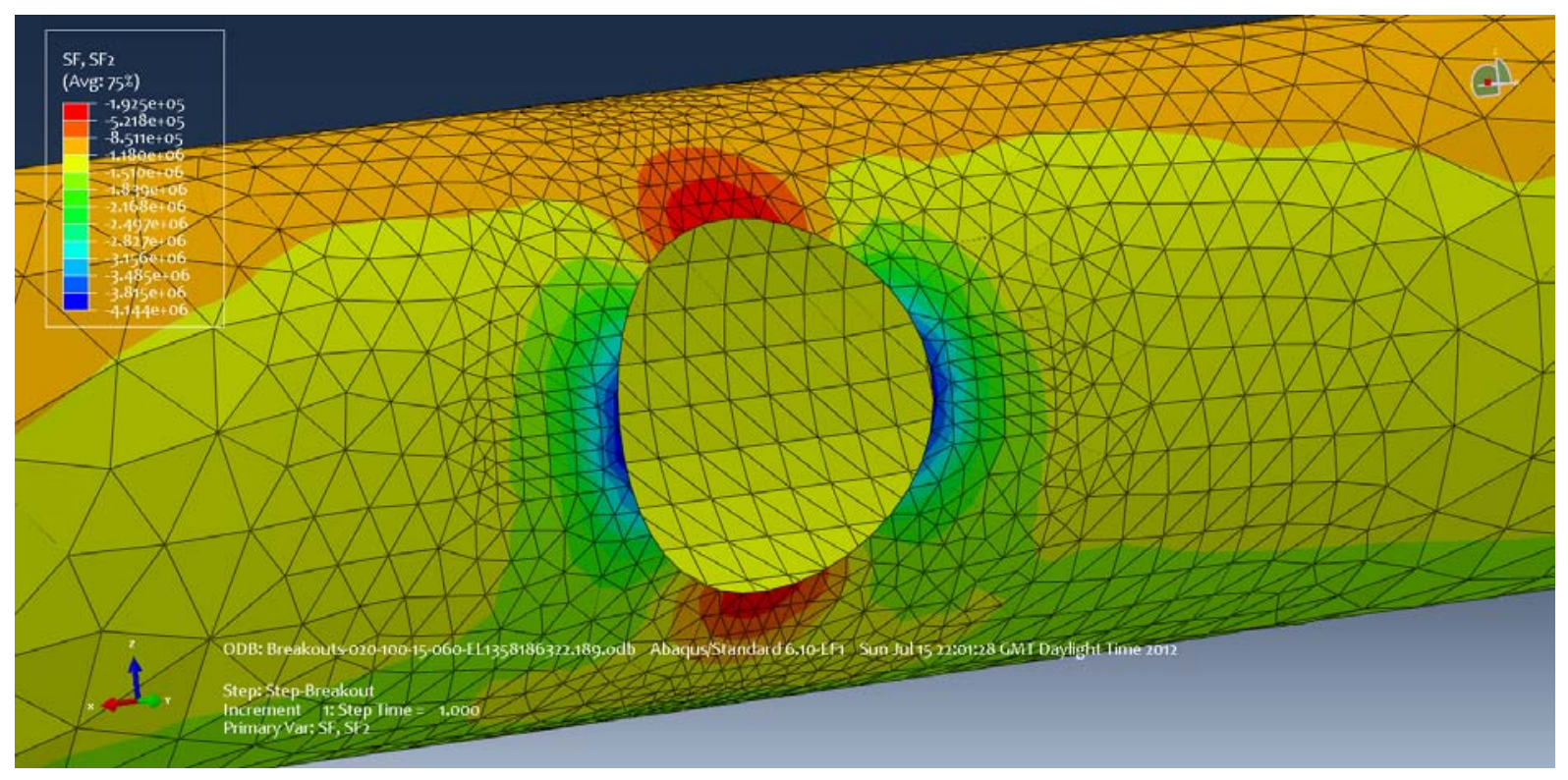

Figure 55: Model Nr.39: 3D-100-15-060; SF2: Hoop forces 


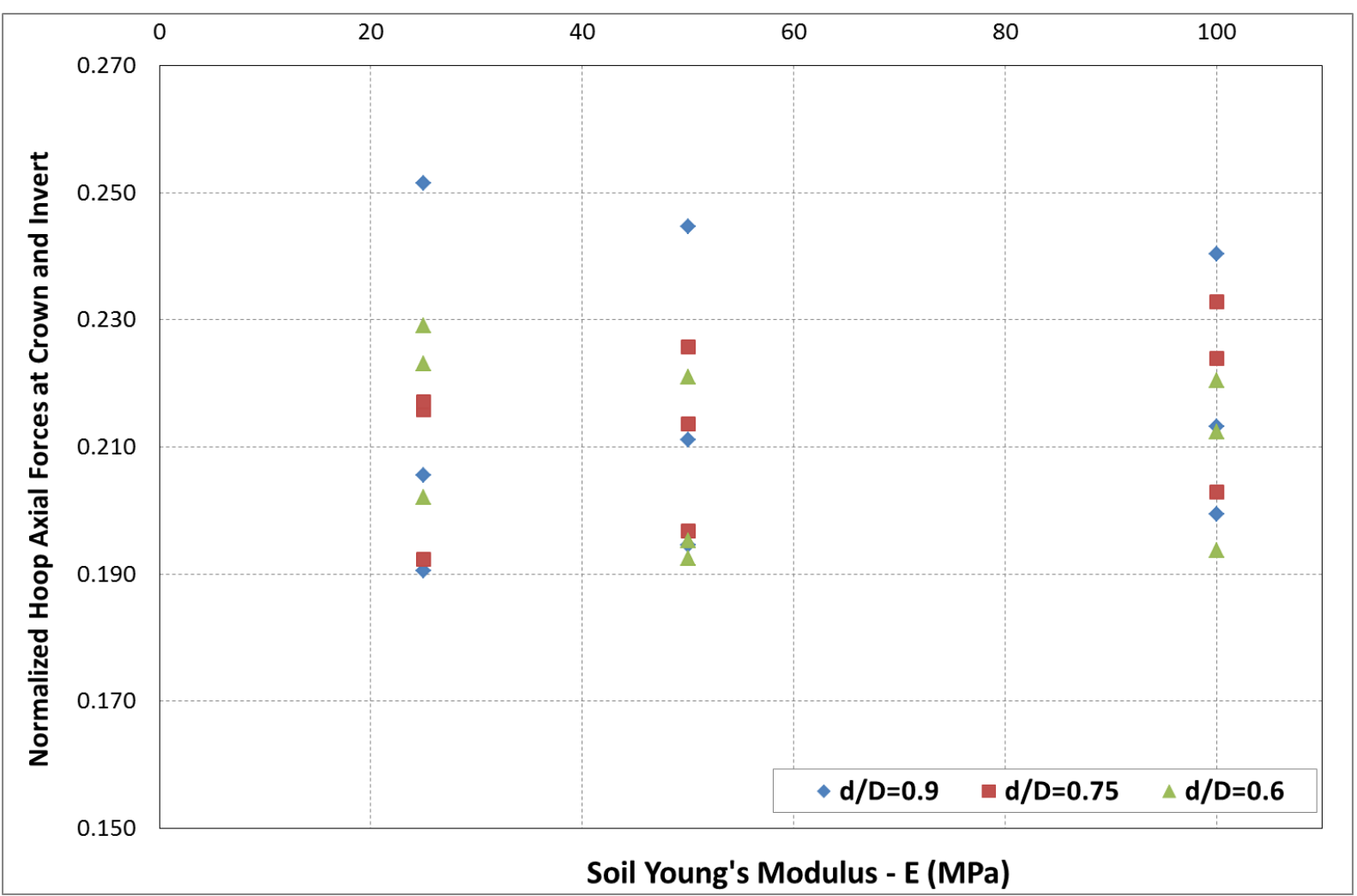

Figure 56: Influence of soil Young's modulus on Hoop Forces at Crown/Invert (sf2-Cl-norm)

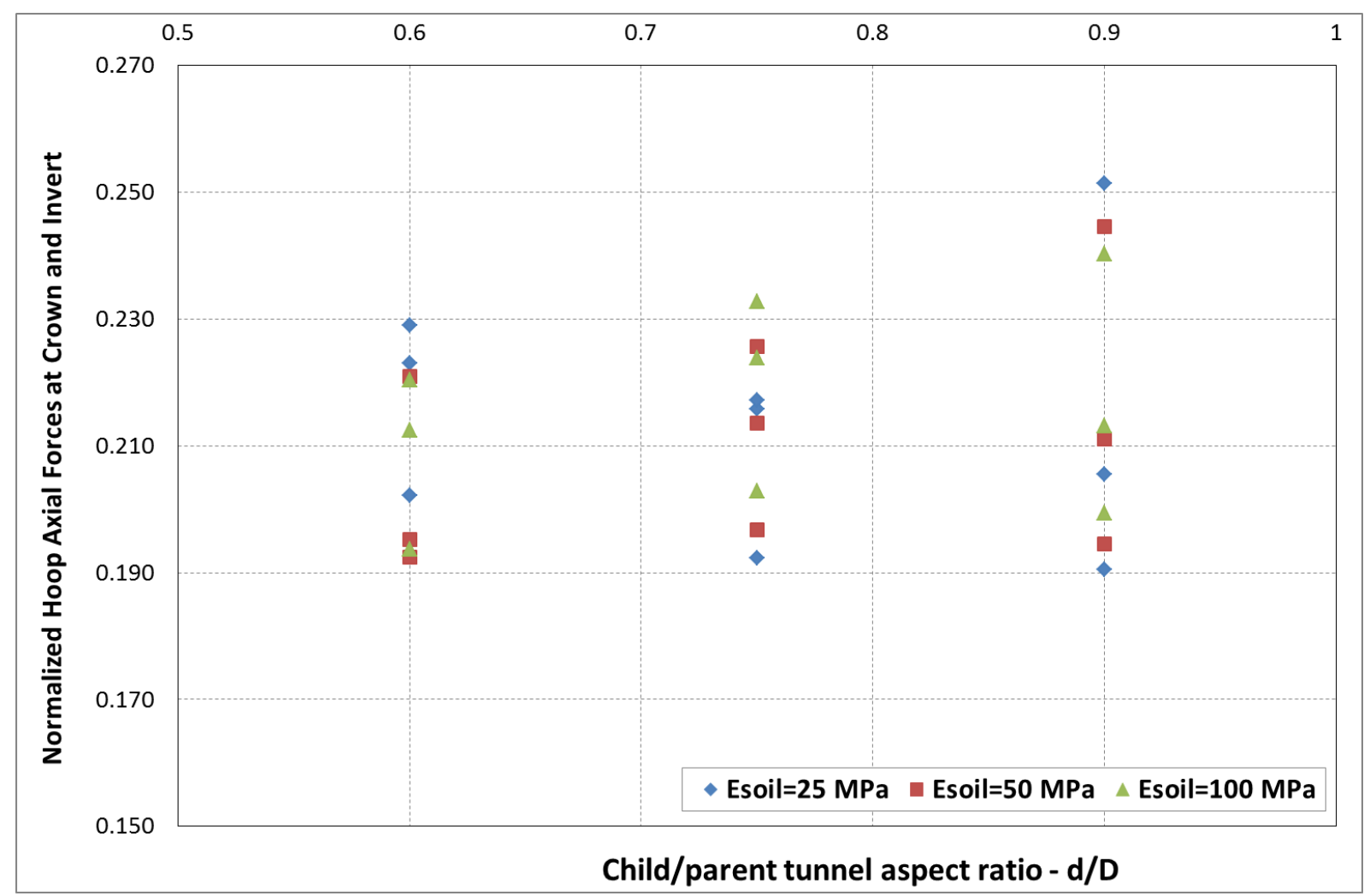

Figure 57: Influence of child-parent tunnel aspect ratio on Hoop Forces at Crown/Invert (sf2-Cl-norm) 


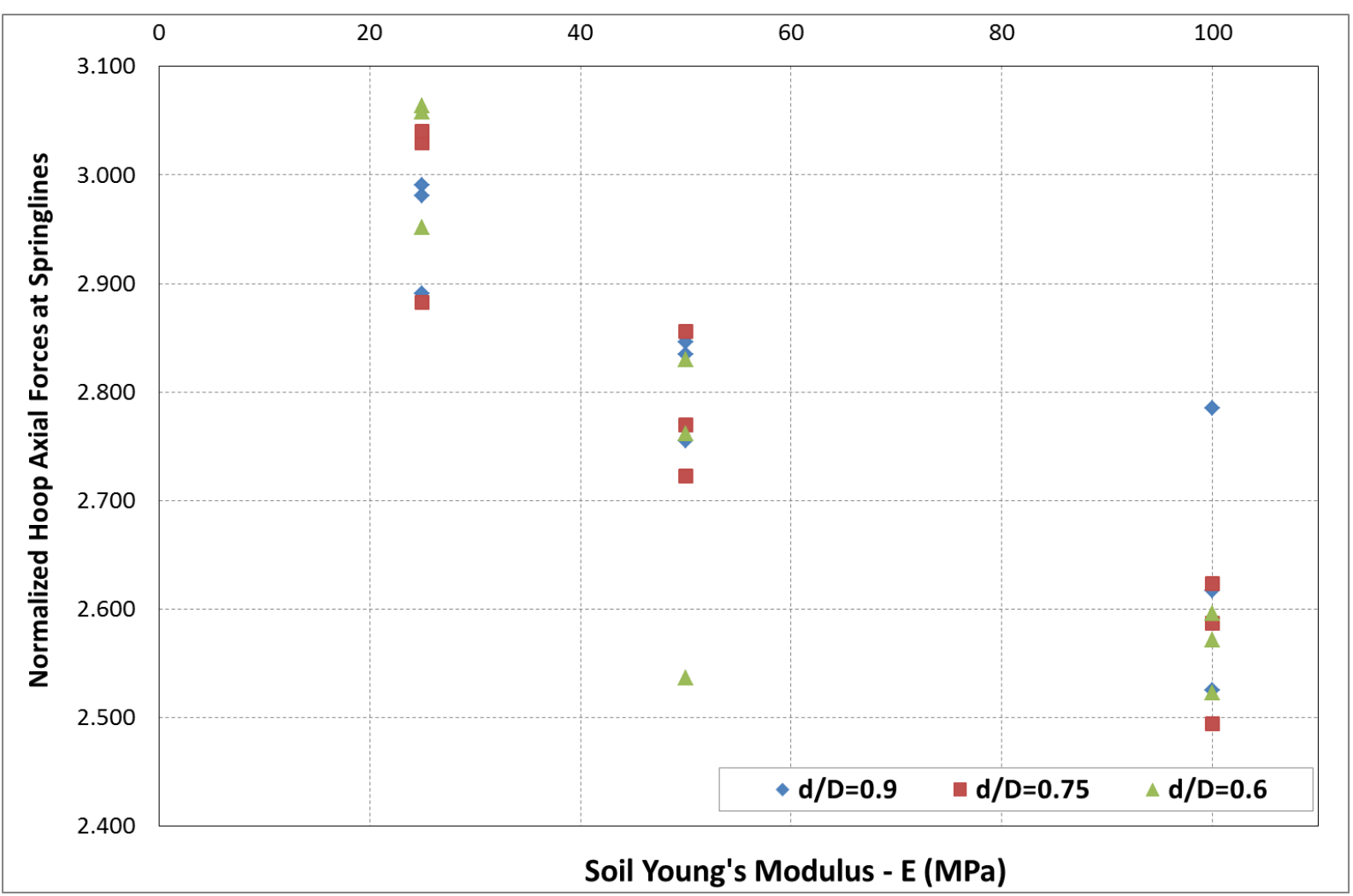

Figure 58: Influence of soil Young's modulus on Hoop Forces at Springlines (sf2-SP-norm)

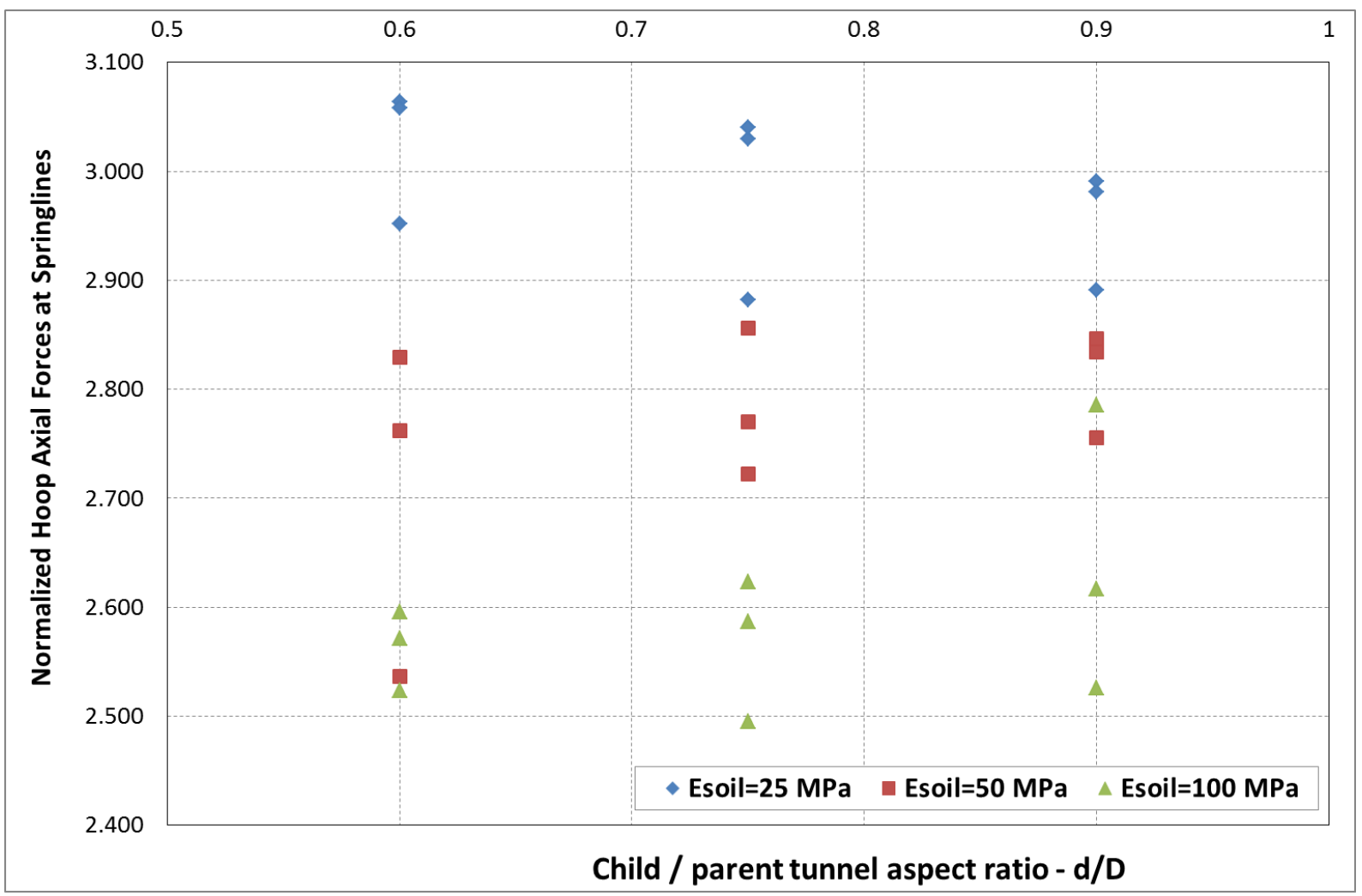

Figure 59: Influence of child-parent tunnel aspect ratio on Hoop Forces at Springlines (sf2-SP-norm) 
The longitudinal moments at crown/invert strongly depend on the surrounding material stiffness, and less on the child tunnel size (Figure 63 - Figure 64): The crown/invert area tends to bend toward the inside, i.e. the intrados comes to a tension state. This effect tends to diminish substantially for higher stiffness of the surrounding soil (higher soil Young's modulus). The influence of the Young's modulus appears to be linearly proportional in the existing cases $\left(N L F \approx 0.008 \cdot E_{\text {soil }}-0.1\right)$. These moments do also vary in an inversely linear manner with respect to the size of the child tunnel opening.

The longitudinal moments at springlines strongly depend on the surrounding material stiffness (Figure 65 - Figure 66): The springlines area tends to bend toward the outside, i.e. the extrados comes to a tension state and pushes directly against the surrounding material. The longitudinal moments at springlines are mainly dependent to the confinement of the lining, which can also be understood as the direct resistance of the soil to the expansion, "pushing" response of the lining at this location. These moments appear to be virtually insensitive to the child tunnel size $(d / D)$ for the investigated cases.

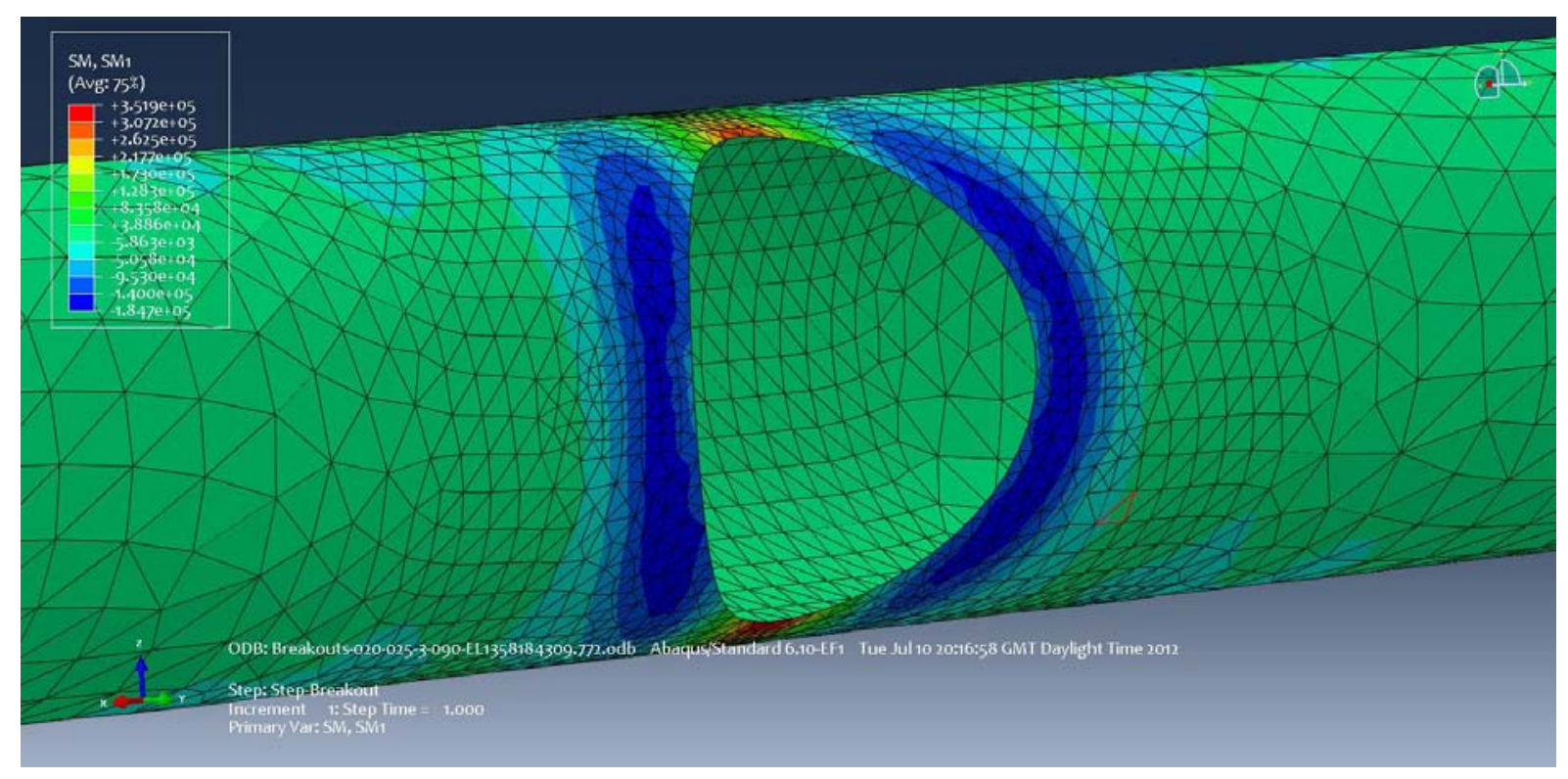

Figure 60: Model Nr.1: 3D-25-35-090; SM1: Longitudinal moments 


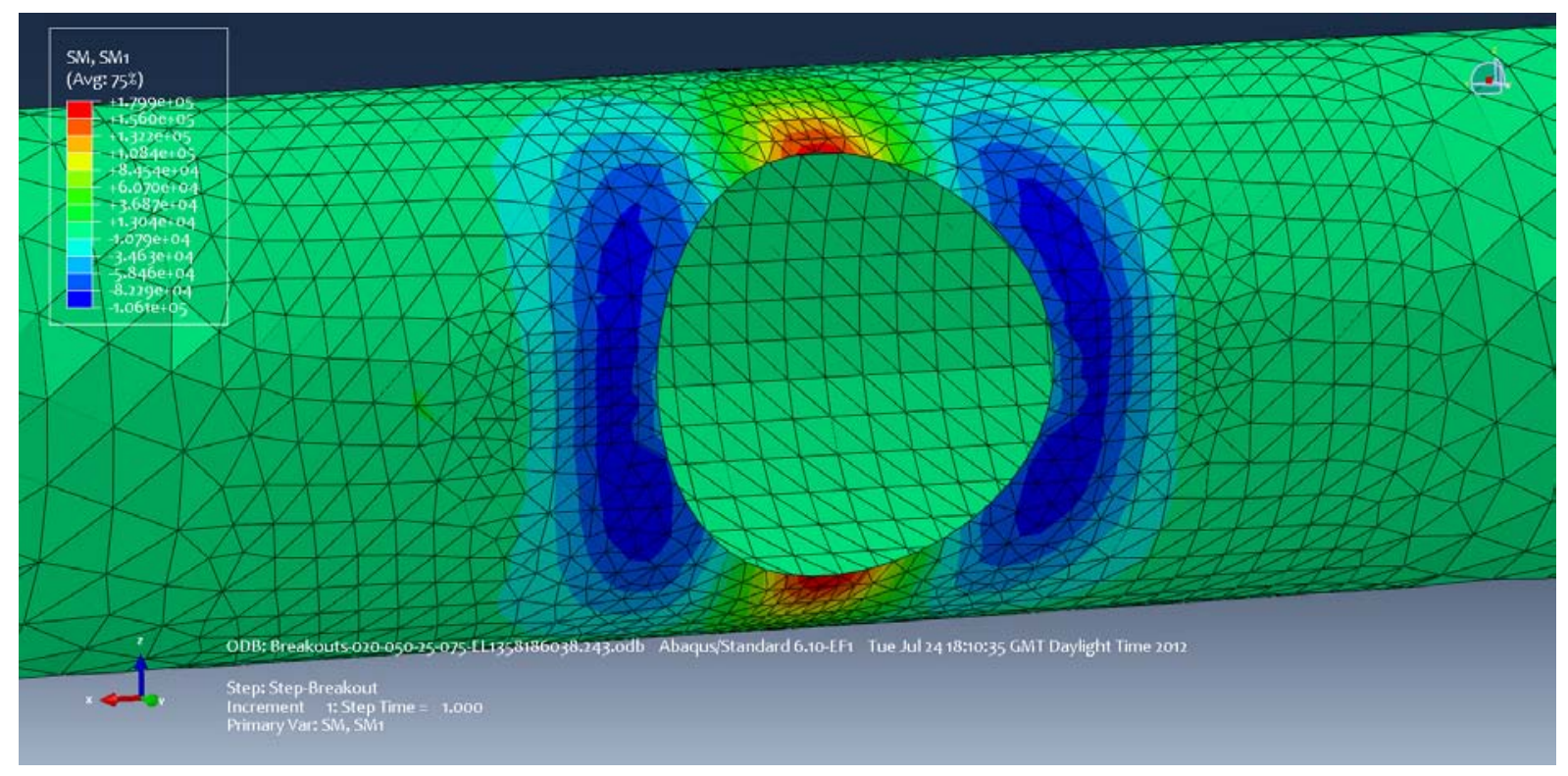

Figure 61: Model Nr.20: 3D-50-25-075; SM1: Longitudinal moments

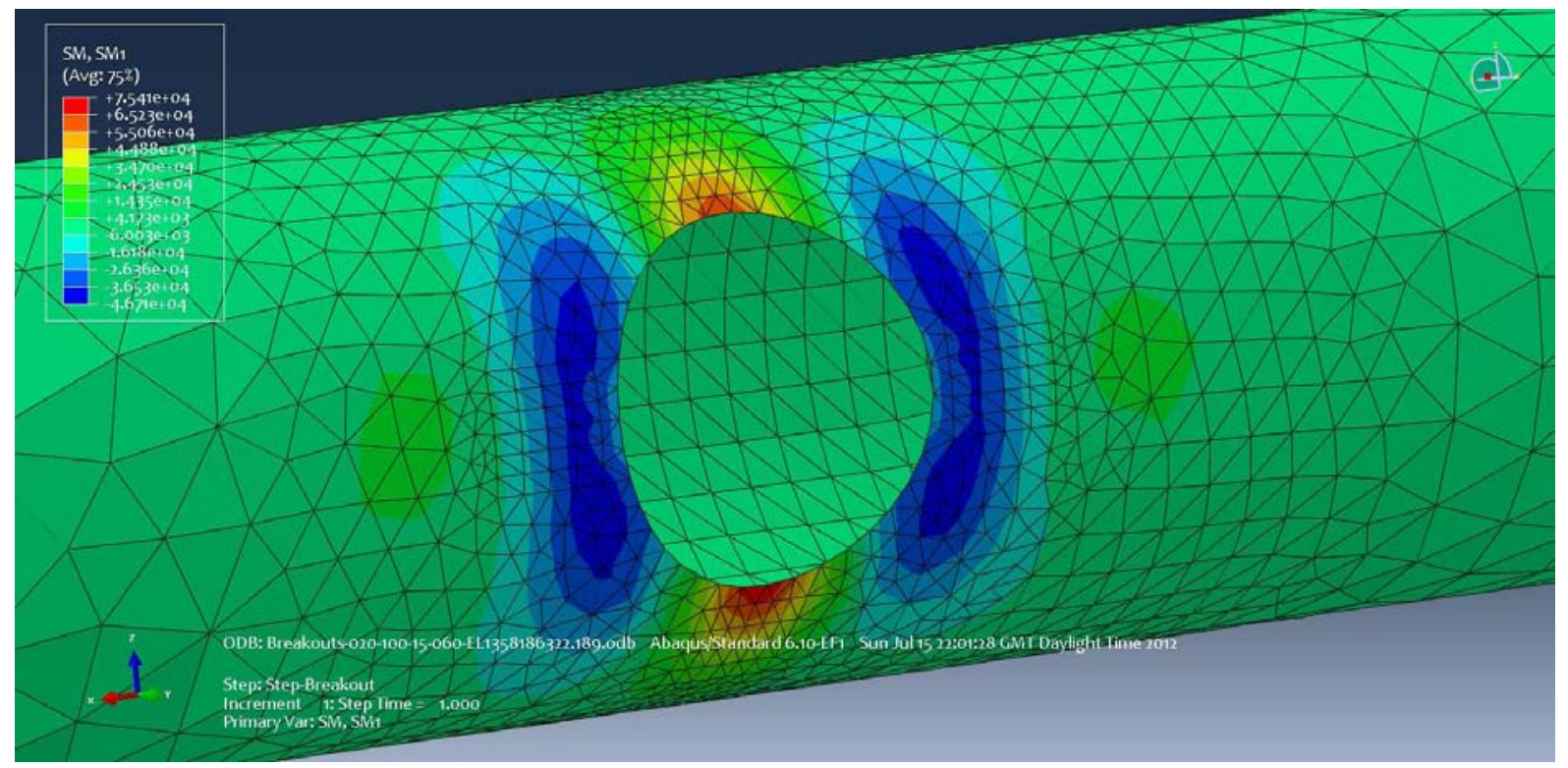

Figure 62: Model Nr.39: 3D-100-15-060; SM1: Longitudinal moments 


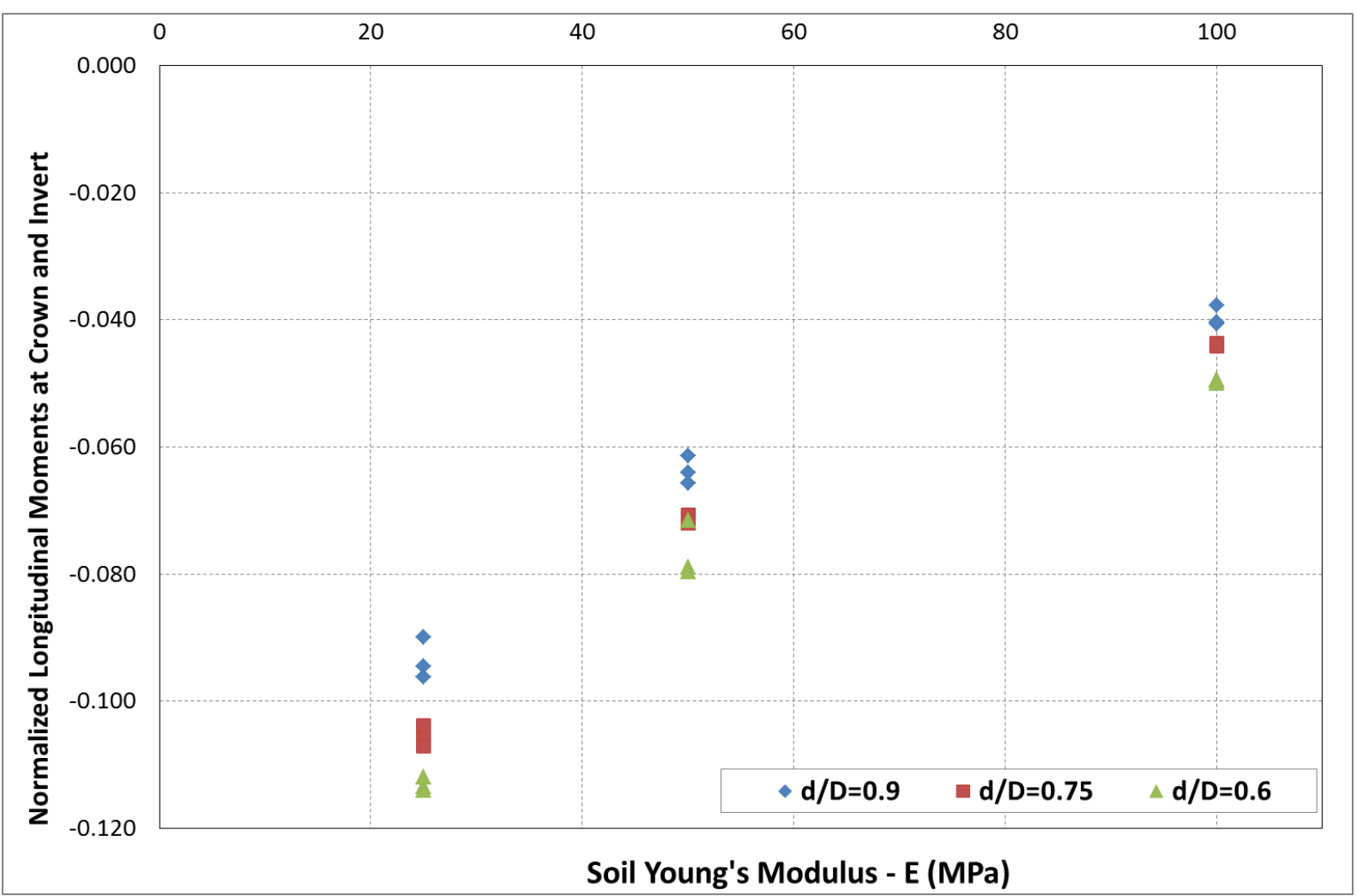

Figure 63: Influence of soil Young's modulus on Longitudinal Moments at Crown/Invert (sm1-Cl-norm)

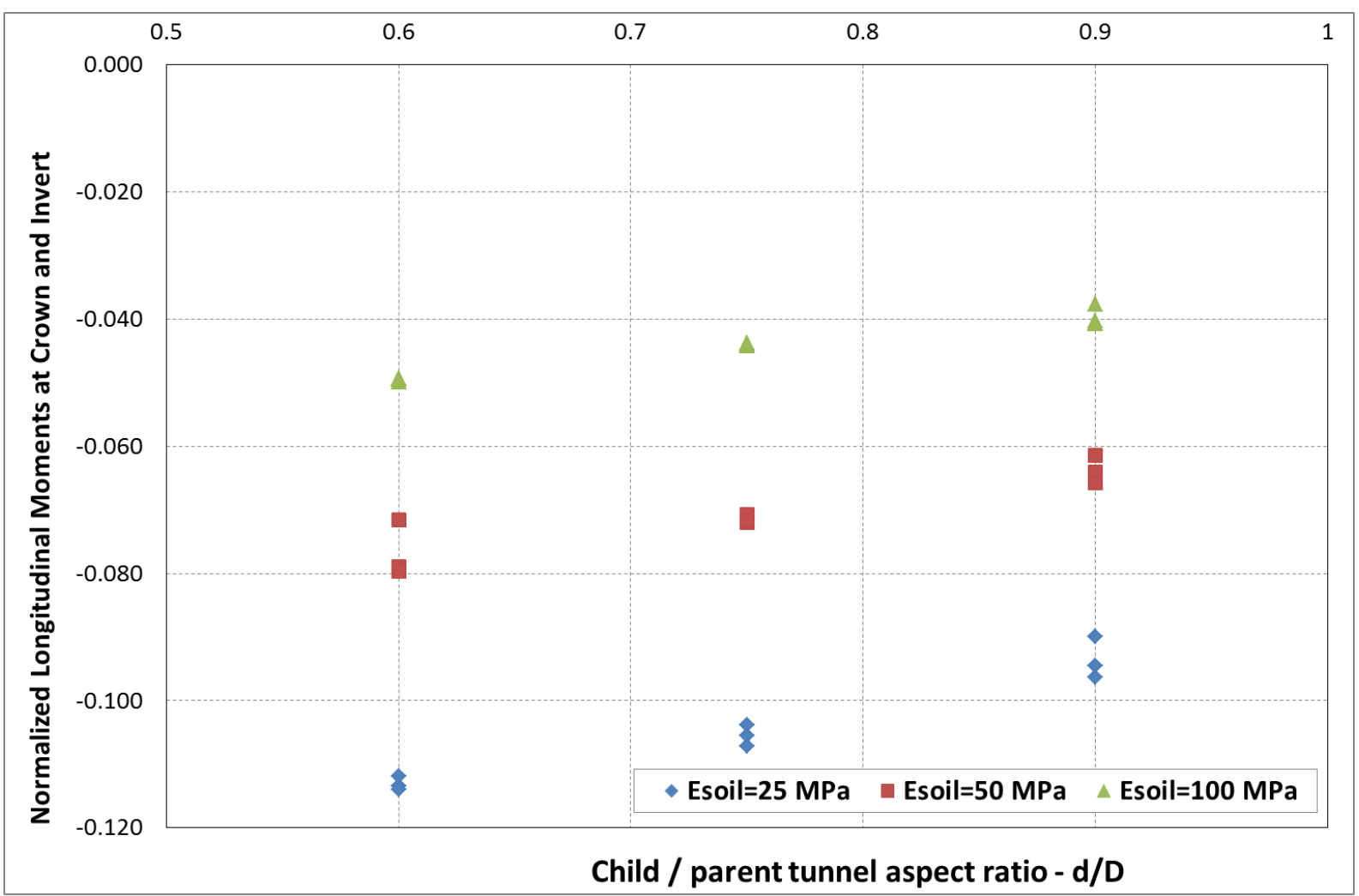

Figure 64: Influence of tunnel aspect ratio on Longitudinal Moments at Crown/Invert (sm1-Cl-norm) 


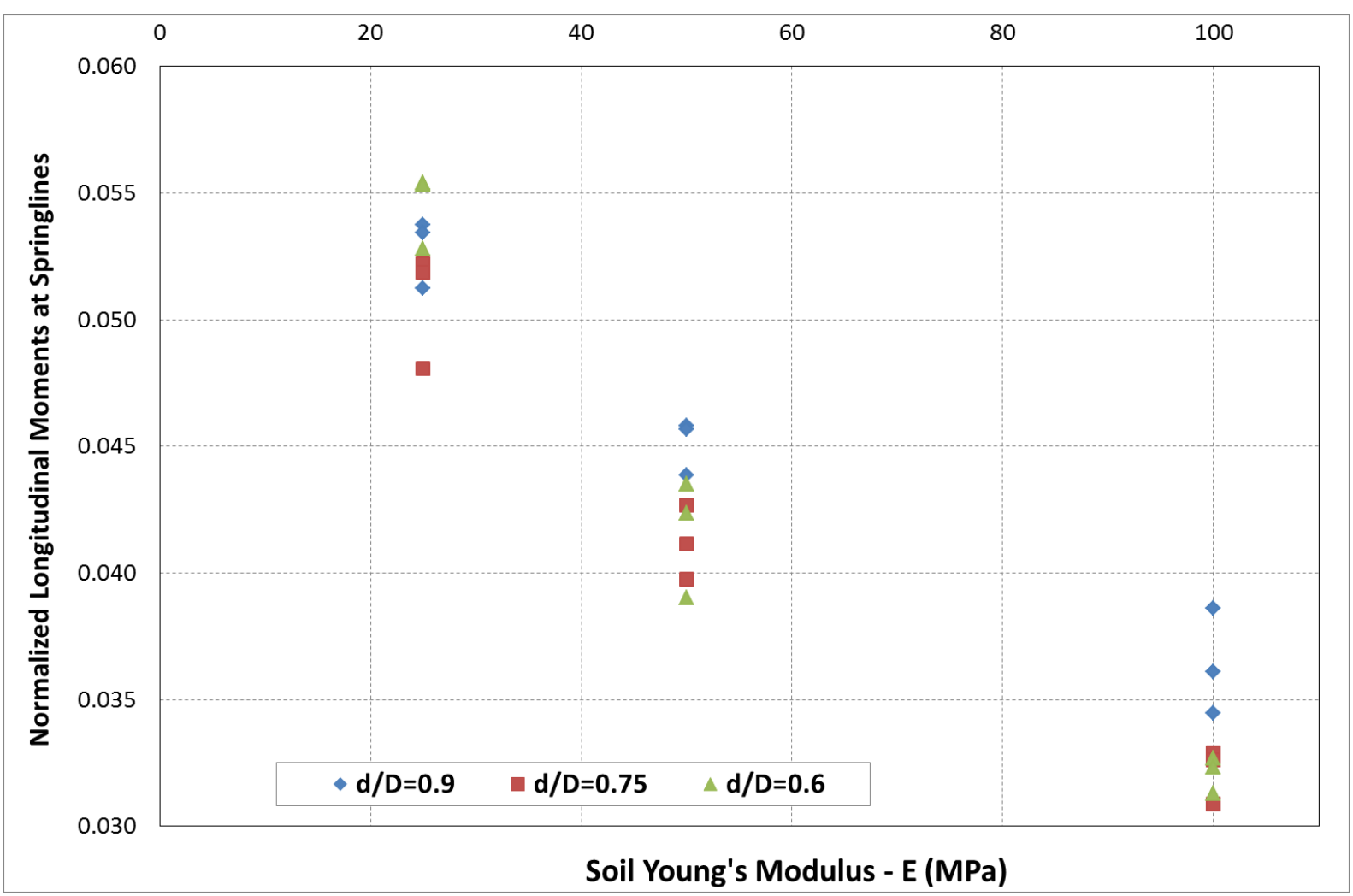

Figure 65: Influence of soil Young's modulus on Longitudinal Moments at Springlines (sm1-SP-norm)

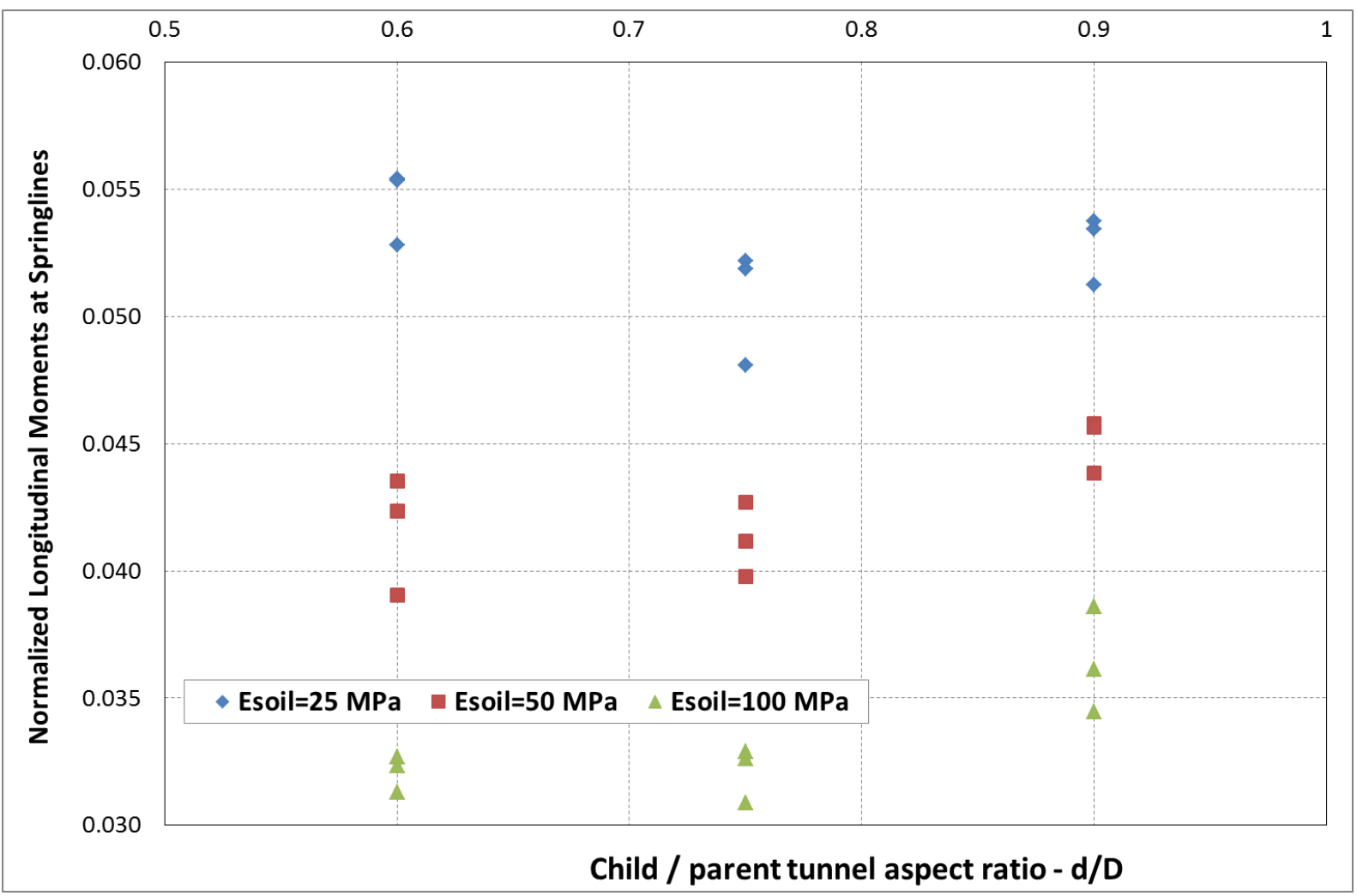

Figure 66: Influence of child-parent tunnel aspect ratio on Longitudinal Forces at Springlines (sm1-SP-norm) 
The hoop moments at crown/invert show strong dependence on the surrounding material stiffness, and some less pronounced influence by the child tunnel size (Figure 70 Figure 71): Bending in the hoop direction in the $\mathrm{Cl}$ area diminishes by half as the soil stiffness is quadrupled, and generally the moments appear to substantially decrease as confinement of the lining increases. At the same time a slight increase in hoop moments appears for larger breakout openings (d/D).

The hoop moments at springlines reflect a substantial influence by the surrounding material stiffness, as well as the child tunnel size (Figure 72 - Figure 73): At the sides too, the larger the confinement the smaller the bending response of the lining. This presents itself as a strong influence, since the moments appear to decrease to less than $30 \%$ for $E_{\text {soil }}$ varying from 25 to $100 \mathrm{MPa}$. The size of the opening seems to affect the bending response of the opening's springlines in an inversely proportional manner, i.e. for large openings, the bending effects decrease.

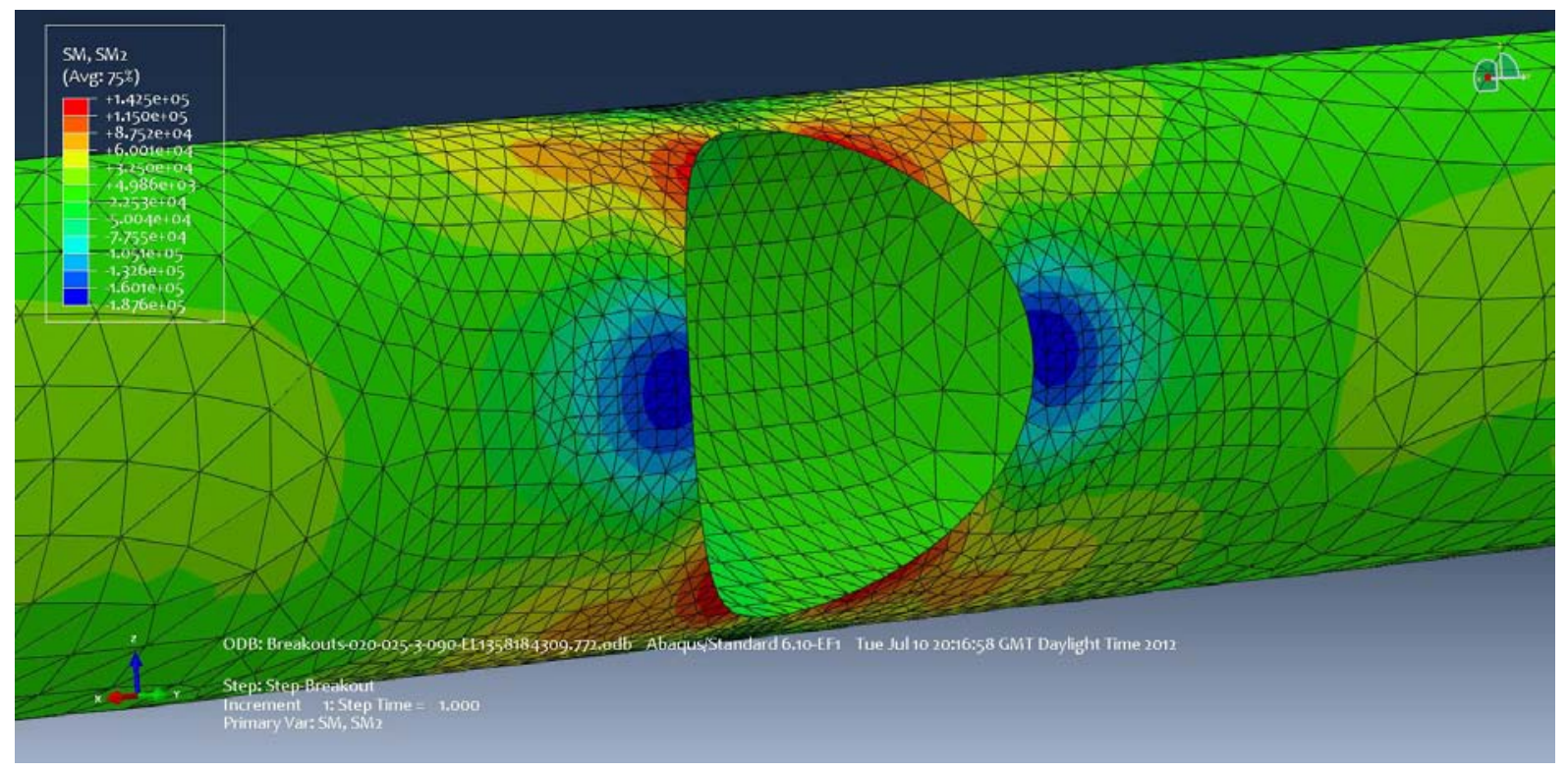

Figure 67: Model Nr.1: 3D-25-35-090; SM2: Hoop moments 


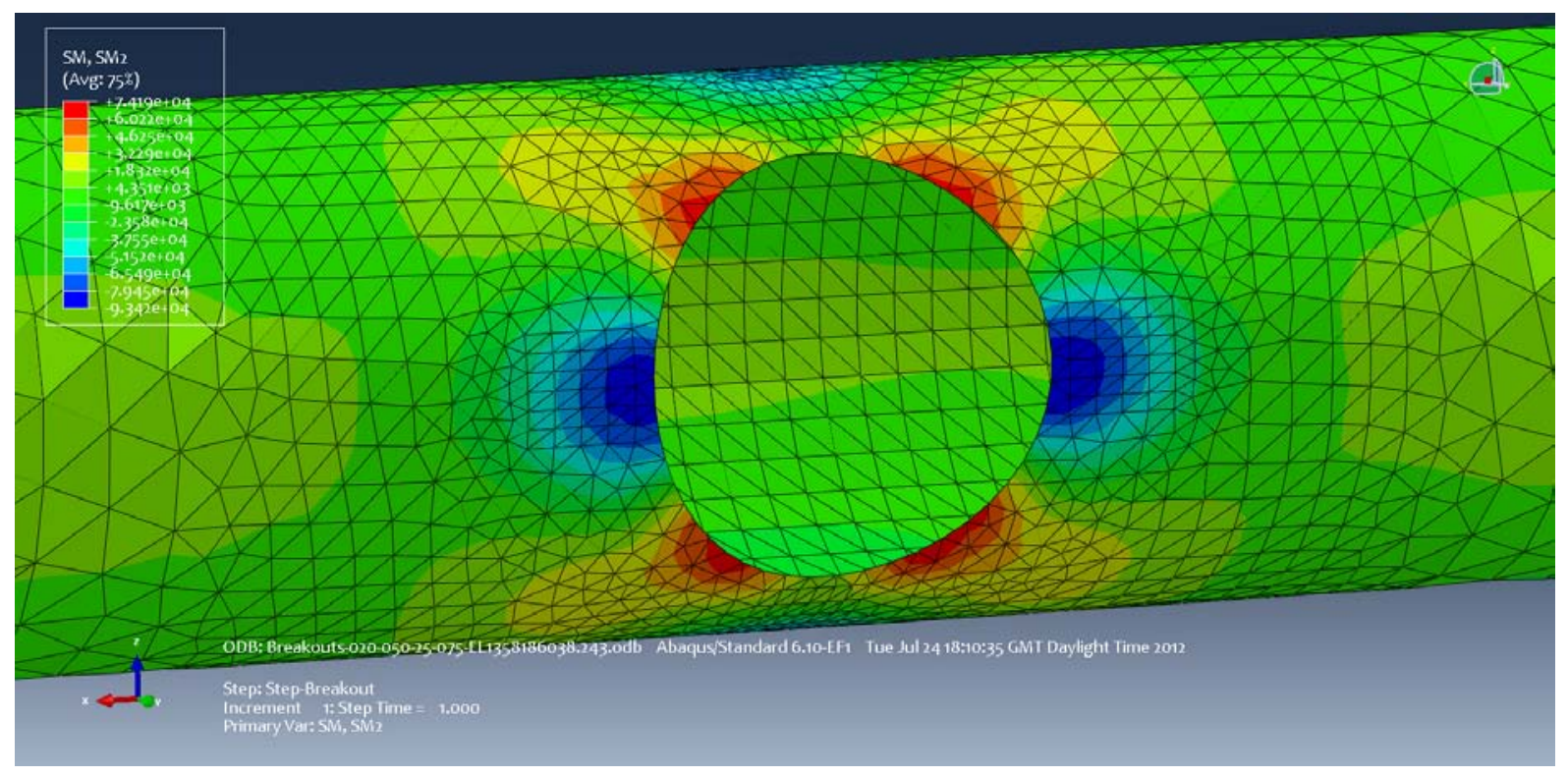

Figure 68: Model Nr.20: 3D-50-25-075; SM2: Hoop moments

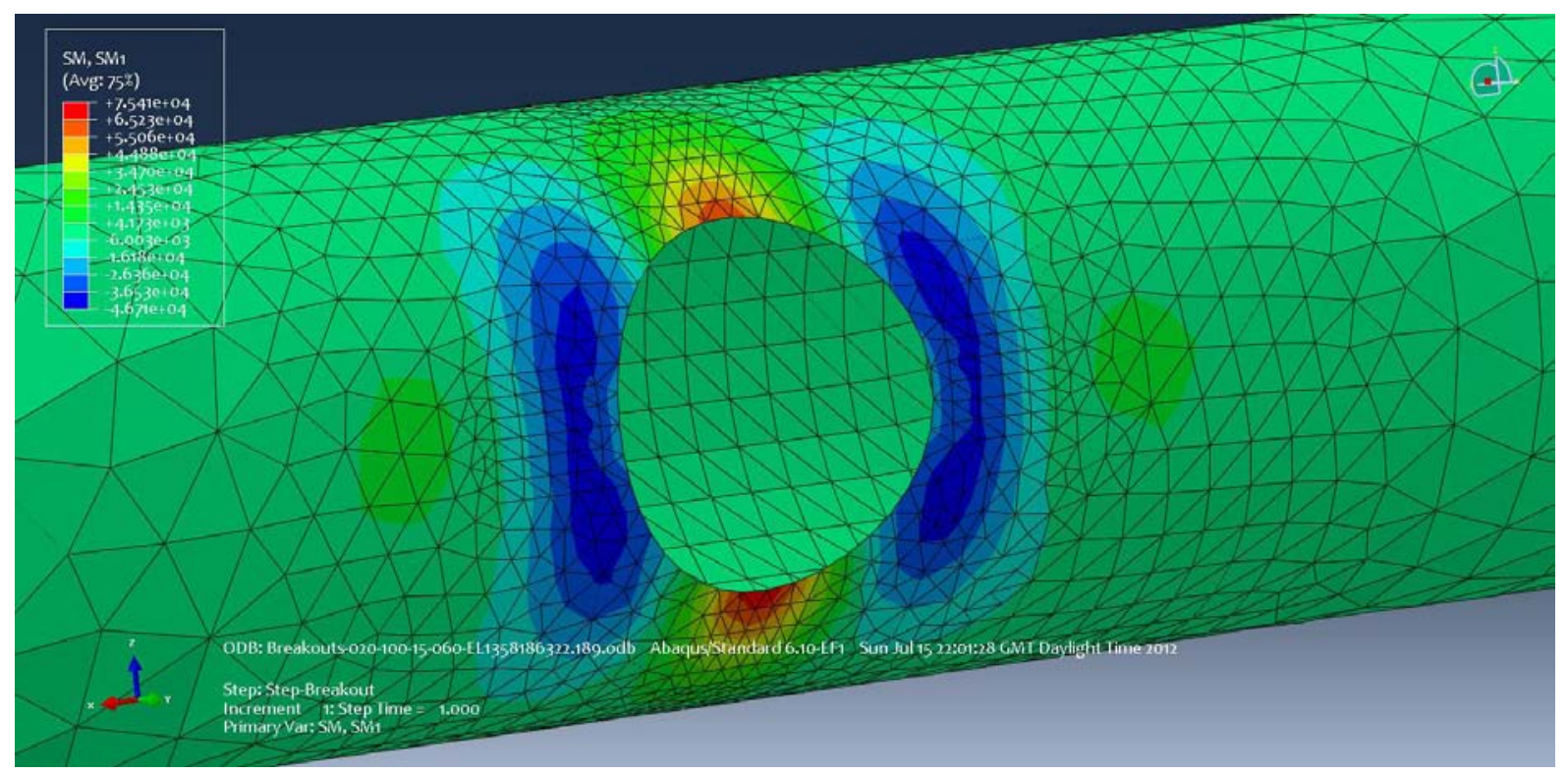

Figure 69: Model Nr.39: 3D-100-15-060; SM2: Hoop moments 


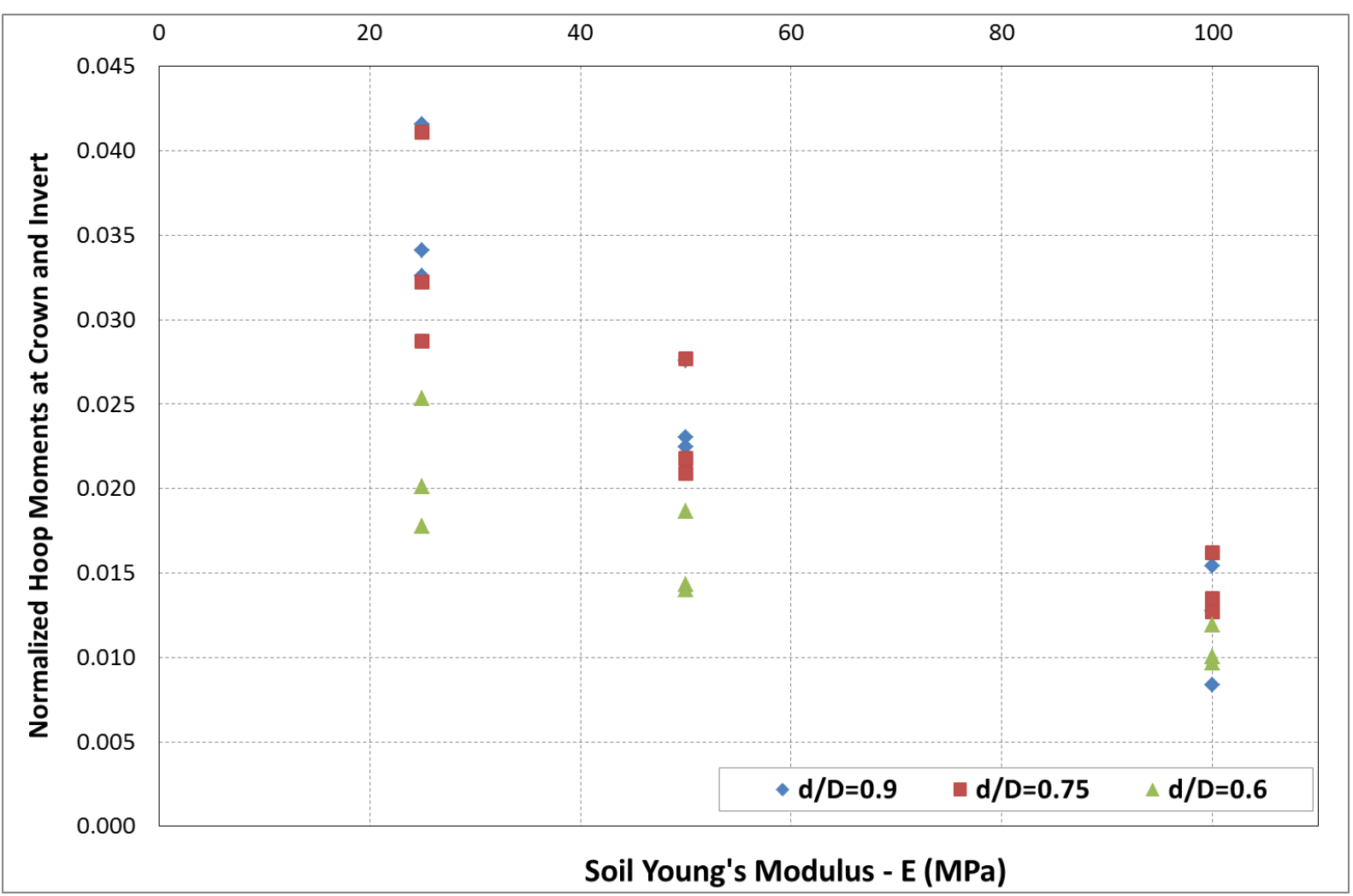

Figure 70: Influence of soil Young's modulus on Hoop Moments at Crown/Invert (sm2-Cl-norm)

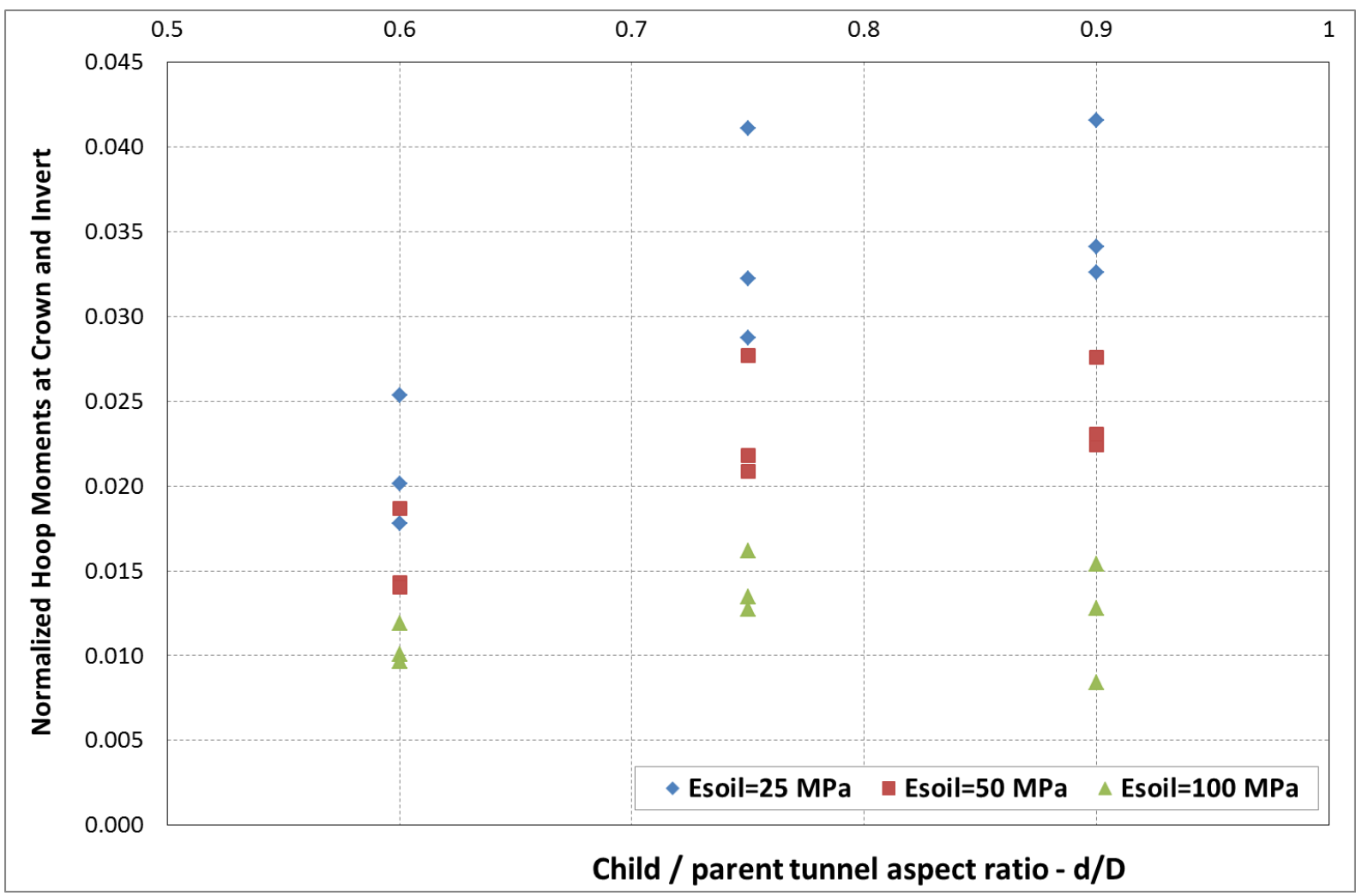

Figure 71: Influence of child-parent tunnel aspect ratio on Hoop Moments at Crown/Invert (sm2-Cl-norm) 


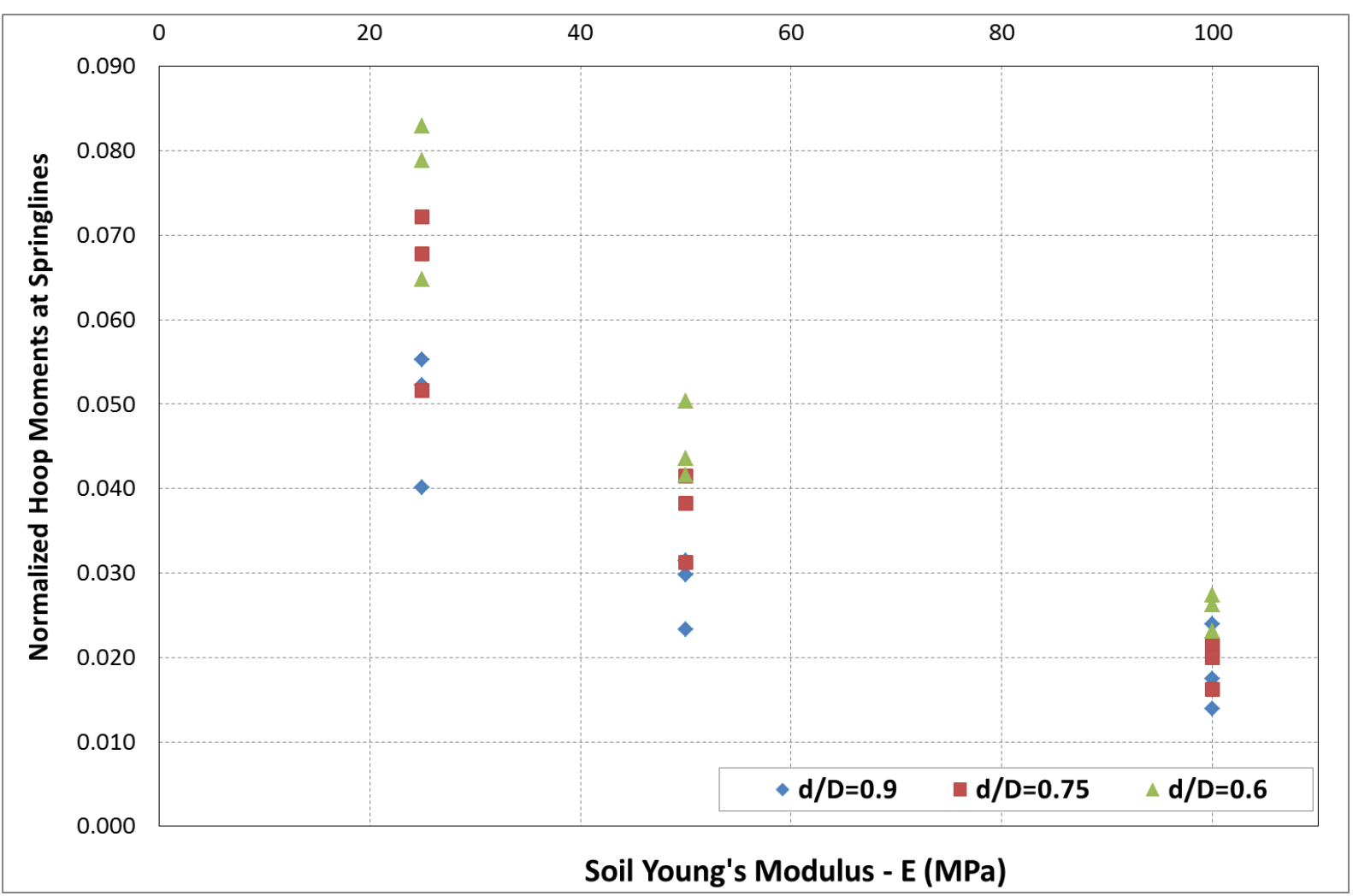

Figure 72: Influence of soil Young's modulus on Hoop Moments at Springlines (sm2-SP-norm)

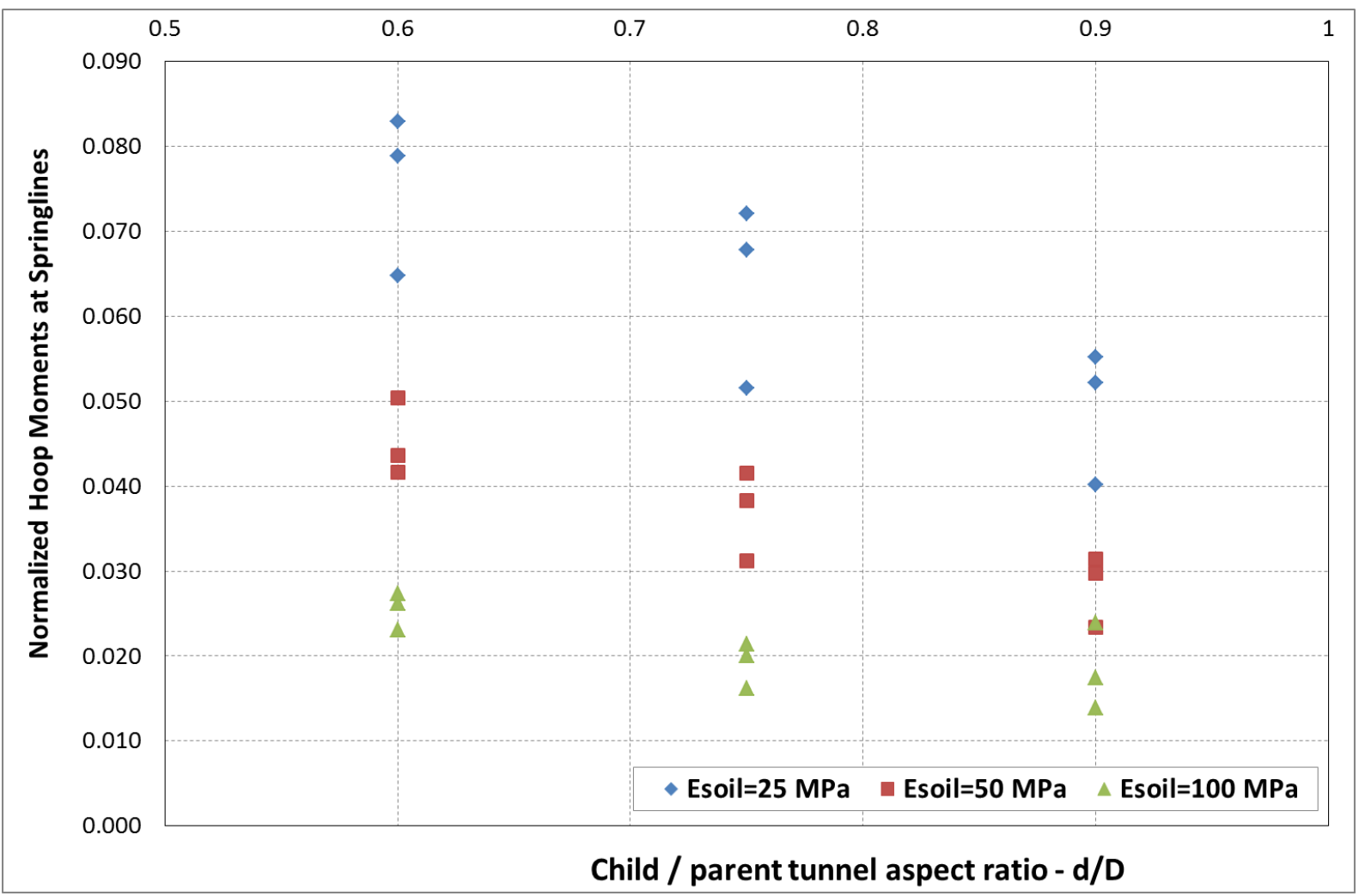

Figure 73: Influence of child-parent tunnel aspect ratio on Hoop Forces at Springlines (sm2-SP-norm) 
The table below summarizes the influences of the checked parameters graphically.

\begin{tabular}{|c|c|c|c|c|c|c|c|c|}
\hline & $\begin{array}{c}\text { Hoop Force } \\
\text { Crown/invert }\end{array}$ & $\begin{array}{c}\text { Hoop Moment } \\
\text { Crown/invert }\end{array}$ & $\begin{array}{c}\text { Long. Force } \\
\text { Crown/invert }\end{array}$ & $\begin{array}{c}\text { Long. Moment } \\
\text { Crown/invert }\end{array}$ & $\begin{array}{c}\text { Hoop Force } \\
\text { Springlines }\end{array}$ & $\begin{array}{c}\text { Hoop Moment } \\
\text { Springlines }\end{array}$ & $\begin{array}{c}\text { Long. Force } \\
\text { Springlines }\end{array}$ & $\begin{array}{c}\text { Long. Moment } \\
\text { Springlines }\end{array}$ \\
\hline E-modulus Soil & - & $\searrow$ & $\searrow$ & $\searrow$ & $\searrow$ & $\searrow$ & $\searrow$ & $\searrow$ \\
\hline $\begin{array}{c}\text { Child / Parent } \\
\text { Tun. Diameter (d/D) }\end{array}$ & - & $\nearrow$ & $\searrow$ & $\searrow$ & - & $\searrow$ & $\searrow$ & - \\
\hline
\end{tabular}

Table 7: Graphical summary of the influences of the checked parameters

\subsubsection{Comparisons of 2D and 3D solutions}

Some similarities in the response of openings at tunnels are disclosed in analytical and numerical 2D as well as the numerical 3D model results. The 3D results (see Table 5) lead to the conclusion that for larger openings $(d / D=0.9)$ and greater overburdens, tension appears to diminish, yet in fact it is transferred to other areas of the lining. These areas lie further in a diagonal direction of approximately 45 degrees. This is also identified in the 2D approach (both analytical and numerical). This indicates that for larger openings, the critical areas for the design are not necessarily concentrated around the tight opening perimeter as originally anticipated. The hoop force concentration at the sides lies within the range of 2.5 to 3 times the original hoop forces prior to breakout which falls in close correlation with the 2D results and analytical calculations. This range is similar for the 3D models with some deviations of up to $20 \%$ in models with aspect ratio $d / D=0.6$. However, apart from these similarities, there are significant differences in the output one can get, not only between a 2D and 3D analysis, but even between the different approaches (Kirsch/numerical) to 2D analyses themselves.

Of course the main and most readily seen issue is that $2 \mathrm{D}$ analyses cannot provide any information on the bending response of influence regions around openings. At the same time, the results of 3D models (see Table 5) show substantial values of bending moments that can govern the design.

As for the consistency of the three approaches, i.e. the analytical solution with assumption of circular openings, the $2 \mathrm{D}$ numerical plane stress solution with the accurate developed (unwrapped) geometry of the opening, and the 3D numerical solution, certain deviations appear. The Kirsch solution appears to be all-around the most conservative of the two 2D approaches, but it yields lower compressive forces at the springlines of the openings, 
which is typically governing for the design. Note that this is most probably attributed to the particular shapes of the investigated tunnels and cannot be taken as a generic statement for the comparison of the two solutions.

Beyond that, 3D models yield lower longitudinal forces at crown and invert (also critical for the design), somewhat higher compression forces in the longitudinal direction at the springlines and in the hoop direction at the crown and invert locations, while of course they provide values for the bending moments.

Based on the above findings, 2D models have very limited applicability, and 3D models are anticipated to simulate the stress situation around the openings more realistically. 


\section{Summary}

The present study discusses the structural response of tunnels when a circular breakout is performed laterally at their lining. In the first part, this thesis is set in its context, by providing some main concepts of tunneling design and construction, aspects of numerical modeling with focus on underground structures, analytical solutions for openings in shells/plates from literature, and some main elements with respect to the layout and the descriptive geometry of the idealized investigated structural systems. Furthermore, the investigated systems and the description of the investigation methods are provided, these mainly being linear-elastic finite elements analyses, yet with inclusion of analytical solutions for reference and with some analyses assuming non-linear soil behavior in order to investigate particular aspects in the discussed systems. This thesis comes to contribute to the little so far available in literature with respect to the soil structure-interaction and the structural behavior at tunnel breakouts and junctions, while certain reference is given for future similar design through 35 analyzed cases.

Intention and scope of this thesis includes (a) to indicate design and construction aspects of the focused engineering problem (b) to suggest a modeling procedure for the particular problem, (c) to present the sensitivities of the respective two- and threedimensional analytical and FE analyses with strategically selected input-parameters, and (d) to discuss the pitfalls and benefits of these approaches and provide feedback for the engineering analysis of lateral openings in typical tunnels. The basis of the study comprises mainly numerical calculations, with reference to analytical solutions. It should be noted that the main background of the study reflects the sprayed concrete tunneling method (NATM or similar, as opposed to segmental lining tunnels through boring machines), yet the general concepts discussed herein are anticipated to apply to other types of underground structures as well.

The investigated items are limited to cases pertaining to tunnel structures with the following characteristics:

- Circular sections for both the parent and the child tunnel 
- Soft substrate, and soil material envisaging shallow tunnels with a nearisotropic confining stress state $\left(K_{0}=1\right)$

- Elastic behavior of both the soil and the lining material in most cases.

Still, it is anticipated that this work covers a wide range of tunneling systems relevant to practice and advances the general understanding of their behavior.

\subsection{Main conclusions and recommendations for design}

Throughout this thesis, certain conclusions may be extracted:

1. Kirsch solutions (or similar) may prove to be inadequate as a design tool for non-circular openings, as they yield a lower compression at the springlines and higher values in all other cases. If the opening layout is of a shape that allows for a plane-stress analytical approach, it is recommended to calculate with the assumption of different shapes and sizes of a tunnel to create an envelope of results, or in other words to perform a sensitivity-analysis. Using the Kirsch solution for a junction of two approximately circular tunnels it is recommended to perform the Kirsch solution for an opening with diameter equal to

i. the child tunnel diameter (red line in Figure 74) and

ii. the arch length of the perpendicular projection of the child tunnel's circumference on the curved parent tunnel surface (blue line in Figure 74).

2. Two-dimensional analyses are particularly sensitive to the shape of the opening. In that sense two dimensional numerical models should be preferred to analytical solutions, provided that the developed geometry of the opening is well modeled; reasonable models for such junctions' analysis should account for the actual projected perimeter of the child tunnel on the parent tunnels curved surface. User (and budget) friendly CAD plug-ins are available and can assist in modeling such geometries. 


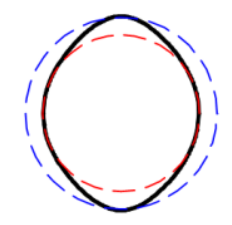

Figure 74: Development of a tunnel with opening, and envelope of circular opening shapes to be assessed through analytical methods (e.g. Kirsch)

3. Certain dependencies have been identified between the stress redistribution and the problem parameters, as for example the tunnel depth, the child/parent tunnels aspect ratio, the surrounding soil's Young's modulus - and consequently the confinement conditions of the tunnel. These dependencies are demonstrated in the results of this study. Still, this study is primarily comparative and focuses on simple geometries; therefore it is deliberately avoided herein to present strict conclusions on any mathematical relationships between the input parameters and the quantified stress redistributions. It can however be generally understood from the results that the forces around openings are distributed as follows:

i. Significant tension and positive moments (extrados in compression - intrados in tension) develop in the longitudinal direction at the crown and the invert of the opening. These are to be borne with a tension bearing mechanism (e.g. rebar reinforcement) closer to the internal face of the lining.

ii. Very high compression and negative moments (extrados in tension - intrados in compression) develop in the hoop direction at the openings springlines (sides). These may be borne by sufficient thickening on either side which increases both the compressive and the flexural capacity. This thickening is preferably installed (e.g. sprayed), after ring closure and loading of the initial tunnel support, because a thickened section of the tunnel in its initial condition may attract 
higher hoop forces and increase stresses in these locations around the opening even before the breakout is realized.

iii. Considerable moments and tensile stresses may appear in the springlineslongitudinal direction and the crown/invert-hoop direction; these also need to be examined in the design, as they often prove to be relevant for the dimensioning of the structure.

iv. Tensile stresses may appear in the parent tunnel lining further from the opening perimeter, in a diagonal pattern, i.e. left and right from the crown, and above and below the springlines. These may also prove to be critical for the design and must be examined in the respective structural calculations.

v. The regions around the opening that are affected by redistribution of stresses extend to approximately one diameter of the child tunnel around the opening.

4. It is being evident, mainly from the $3 \mathrm{D}$ analysis results, that the sizes of the openings and the soil stiffness have a strong influence on the stress redistributions after the formation of the opening. The present study indicates that, out of the two, the soil stiffness has a more substantial effect on the breakout's structural response.

5. The results also show that the stress levels of the lining prior to breakout influence the pattern of the stress redistribution. This is mainly attributed to the confinement of the tunnel by the surrounding material, which for shallow tunnels is affected not only by the soil stiffness, but also by the depth of the soil above the tunnel, i.e. the overburden (note, the stress in the lining at ring closure is essentially proportional to the overburden). Inversely regarded, breakouts may influence the tunneling induced settlements.

6. Since the structural response of the tunnel lining around the opening appears to be particularly sensitive to the confinement of the surrounding material (this is mainly identified as a sensitivity of the results to the soil stiffness), a strong soil-structure interaction process appears. Moreover, the lining shell around the junction exhibits a certain three dimensional response (e.g. bending moments out of the shell's plane). In order to capture this behavior, it is recommended to implement 3D numerical tools in the analysis and design of such elements. 


\subsection{Further investigations}

Based on the experience gained by the author from the present study and his practical involvement in tunneling projects, the following directions are given for further relevant investigations. These should pursue:

1. To identify the response for the openings at junctions of various shapes of parent and child tunnels. This should also include various geometric layouts of the junctions, e.g. multiple openings in parallel and in vicinity, or openings on both sides of the parent tunnel.

2. To capture the dependencies of the opening response on different soil properties. In particular it is expected that the soil compressibility, and hence the Poisson's ratio may have an important effect on the tunnel confinement around the opening. In addition, variation of the soil confinement through variation of the lateral pressure coefficient, $\mathrm{K}_{0}$ (herein assumed equal to 1 ) should be considered. At a second stage the non-linear material properties of the surrounding medium and their influences can be examined.

3. A better replication of the various construction sequences of such elements, exemplarily given:

a. staged breakout (top-heading, bench-invert),

b. strengthening of the lining prior to breakout, or

c. breakout followed by an immediate construction of the first ring of the child tunnels in a stiff connection to the parent tunnel.

4. To investigate the efficiency/feasibility of retrofitting techniques and strengthening layouts for junctions at new or existing tunnels, including their effects on the structural characteristics at the breakout. 


\section{References}

[1] Bergmeister K; Breen JE; Jirsa JO; Kreger ME; (1993). Detailing for Structural Concrete. Center for Transportation Research Report 0-1127-3F. University of Texas, Austin.

[2] Bond A; Harris A; (2008). Decoding Eurocode 7. Taylor \& Francis.

[3] Box GEP; Draper NR; (1987). Empirical model-building and response surfaces. John Wiley \& Sons.

[4] British Tunnelling Society; (2004). BTS Tunnel Lining: Design Guide. British Tunnelling Society (BTS), London. Thomas Telford.

[5] Crossrail C121 (2011) Non Linear Numerical Modelling Parameters - Rev 2. London.

[6] Curtis DJ; (1976). Discussions on Muir Wood. The circular tunnel in elastic ground. Geotechnique 26(1).

[7] Dr. Sauer and Partners Ltd.; (2011). Crossrail C121 - Independent Check Work Plan - Whitechapel Station. London.

[8] Dr. Sauer and Partners Ltd.; (2012). Crossrail C121 - Independent Check Work Plan - Fisher Street Shaft. London

[9] Duddeck H; Erdmann J; (1985). Structural design models for tunnels in soft soil. Underground Space (9)1.

[10] Feiersinger A; Mitsch T; Spyridis P; (2012). Preservation of structural and functional integrity in the interaction of new and existing structures: the case of London Underground's Green Park station. In: Life-Cycle and Sustainability of Civil Infrastructure Systems: Proceedings of the third International Symposium on LifeCycle Civil Engineering - IALCCE 2012. Vienna. CRC Press.

[11] fib - International Federation for Structural Concrete; (2012). Model Code 2010 final draft Volumes 1 and 2. Fédération Internationale du Béton (fib) International Federation for Structural Concrete.

[12] Gakis A; Spyridis P; (2011). 222C2 2D FE Aanalyses_8-FE-004 Binocular Tunnel at Fisher Street Crossover Calculation Summary. Internal report, Dr. Sauer \& Partners.

[13] Dassault Systèmes; (2011). Abaqus/standard user's manual v.6.9. Dassault Systèmes

[14] Hight DW; Gasparre A; Nishimura S; Minh NA; Jardine RJ; Coop MR; (2007). Characteristics of the London Clay from the Terminal 5 site at Heathrow Airport. Géotechnique, 57(1), 3-18. 
[15] Hindle D; (2012). Can someone please explain exactly what is SCL? Tunnels and Tunnelling. August/2012.

[16] Hoek E; Brown ET; (1980). Underground Excavations in Rock. London.

[17] Hoek E; Carranza-Torres C; Diederichs M; Corkum B; (2008). The 2008 Kersten Lecture Integration of geotechnical and structural design in tunnelling. In: Proceedings of the University of Minnesota 56th Annual Geotechnical Engineering Conference. Minneapolis.

[18] Hsiao FY; Yu CW; Chern JC; (2005). Modeling the behaviors of the tunnel intersection areas adjacent to the ventilation shafts in the Hsuehshan tunnel. In: Proceedings of the International Symposium on Design, Construction and Operation of Long Tunnels. Taipei.

[19] Hsiao FY; Wang CL; Chern JC; (2009). Numerical simulation of rock deformation for support design in tunnel intersection area. Tunnelling and Underground Space Technology, 24(1).

[20] Jäger J; (2002). Tunnel junction at shallow cover: a case study. In: Proceedings of the third international conference on engineering computational technology. Prague. Civil-Comp press.

[21] Jankowiak T; Lodygowski T; (2005). Identification of parameters of concrete damage plasticity constitutive model. in: Foundations of civil and environmental engineering, 2005(6).

[22] Jones BD; (2007). Stresses in sprayed concrete tunnel junctions. Doctoral thesis, University of Southampton.

[23] Jones BD; (2013). Being analytical. Tunnelling Journal. January/2013.

[24] La Borderie C; Matallah M; (2004). A new concept in damage plasticity coupling, application to fiber reinforced concrete. In: 6th International RILEM Symposium on Fiber Reinforced Concretes, Sarl. RILEM Publications.

[25] Laubbichler J; Schwind T; Urschitz G; (2004) Benchmark for the future: the largest SEM soft ground tunnels in the United States for the Beacon Hill Station in Seattle." In: North American Tunneling 2004: Proceedings of the North American Tunneling Conference 2004, Atlanta, Georgia. Taylor \& Francis.

[26] Lee J; Fenves GL; (1998). Plastic-damage model for cyclic loading of concrete structures. Journal of engineering mechanics, 124(8).

[27] London Underground; (2007). Tottenham Court Road Station Upgrade, Geotechnical interpretative report. London.

[28] Lubliner J; Oliver J; Oller S; Onate E; (1989). A plastic-damage model for concrete. International Journal of Solids and Structures, 25(3). 
[29] Moedlhammer H; (2010) Numerical Methods for Tunnelling using ABAQUS and Investigation of Long-Time-Effects of the Shotcrete Shell and its Impact on the Combined Support System. 2010 Marshall Plan Scholarship Paper, University of Leoben.

[30] Moon H; Lee H; (1991). A 3-D FE Model of a Shaft-tunnel-tunnel Intersection and Comparison With a 2-D Model. In: International Society for Rock Mechanics - 7th ISRM Congress. Aachen, Germany. Balkema

[31] Muir Wood AM; (1975). The circular tunnel in elastic ground. Geotechnique, 25(1).

[32] O'Reilly MP; New BM; (1982). Settlement above tunnels in the United Kingdom their magnitude and prediction. Institution of Mining and Metallurgy, London.

[33] Pilkey WD; Pilkey DF; (2008) Peterson's stress concentration factors. Wiley.

[34] Potts DM; (2003). 42nd Rankine Lecture: Numerical analysis: a virtual dream or practical reality? Géotechnique, 53(6).

[35] Ren G; Smith JV; Tang JW; Xie YM; (2005). Underground excavation shape optimization using an evolutionary procedure. Computers and Geotechnics, 32(2).

[36] Sauer G; Gall V; Bauer E; Dietmaier P; (1994). Design of tunnel concrete linings using limit capacity curves. In: Computer Methods and Advances in Geomechanics, Rotterdam.

[37] Spyridis P; Gakis A; Bedi A; (2013). Numerical Modelling for tunnel structures principles and practicalities. Tunnels and Tunnelling, May/2013.

[38] Spyridis P; Nasekhian A; Skalla G; (2013). Entwurf von Tunnelbauwerken in Spritzbeton-Bauweise am Beispiel London (Design of SCL structures in London). Geomechanics and Tunnelling, 6(1).

[39] Tsagkas GF; (1994). Lessons in Descriptive Geometry - With elements of projection geometry. Thessaloniki. Kyriakidis Bros.

[40] Tsuchiyama S; Hayakawa M; Shinokawa T; Konno H; (1988). Deformation behaviour of the tunnel under the excavation of crossing tunnel. In: Proceedings of the sixth international conference on numerical methods in Geomechanics. Volumes 1-3. Innsbruck, Austria. Balkema.

[41] Tubes Lines Ltd.; (2009). Green Park Station step-free access. Geotechnical interpretative report. London.

[42] Vandewalle L et al.; (2003). RILEM TC 162-TDF: Test and design methods for steel fiber reinforced concrete with the $\sigma-\varepsilon$ design method.Materials and structures, 36(8).

[43] Walter H; (2009). Tunnelstatik - wie zuverlaessig sind die Ergebnisse numerischer Berechungen? Untersuchung anhand eines 3D-Rechenmodells fuer eine 
bergmaennisch vorgetriebene U-Bahnstation (Structural design of tunnels-how reliable are the results of numerical calculations? Investigation using a 3D model for a mined underground railway station). Geomechanics and tunneling Geomechanik und Tunnelbau, 4(4).

[44] Young WC; (1989). Roark's formulas for stress and strain, 6th edition. New York. McGraw-Hill.

[45] Yu CW; Hsiao FY; (2009). Prediction of tunnel behaviour in intersection area. In: ITA-AITES World Tunnel Congress 2009, Budapest.

[46] Yu T; Teng JG; Wong YL; Dong SL; (2010). Finite element modeling of confined concrete-II: Plastic-damage model. Engineering Structures, 32(3).

[47] Zettler A; (2010). Underground excavation design, Lecture Notes. TU Vienna. 